\title{
Friction under active control in systems with tailored degrees of freedom
}

\author{
Dissertation \\ for the award of the degree \\ "Doctor rerum naturalium" (Dr.rer.nat.) \\ of the Georg-August-Universität Göttingen \\ within the doctoral program physics \\ of the Georg-August University School of Science (GAUSS)
}

submitted by

\section{Saeed Amiri}

from Ghorveh, Iran

Göttingen, 2021 


\section{Thesis Committee:}

Dr. Richard LC. Vink

Institut für Materialphysik, Georg-August-Universität Göttingen

Prof. Dr. Peter Blöchl

Institut für Theoretische Physik, TU Clausthal

Institut für Theoretische Physik, Georg-August-Universität Göttingen

PD Dr. Martin Wenderoth

IV. Physikalisches Institut, Georg-August-Universität Göttingen

Members of the Examination Board:

Reviewer: Dr. Richard LC. Vink

Institut für Materialphysik, Georg-August-Universität Göttingen

Second Reviewer: Prof. Dr. Matthias Krüger

Institut für Theoretische Physik, Georg-August-Universität Göttingen

Further members of the Examination Board:

Prof. Dr. Peter Blöchl

Institut für Theoretische Physik, TU Clausthal

Institut für Theoretische Physik, Georg-August-Universität Göttingen

Prof. Dr. Marcus Müller

Institute for Theoretical Physics, Georg-August-Universität Göttingen

Prof. Cynthia A. Volkert, PhD

Institut für Materialphysik, Georg-August-Universität Göttingen

PD Dr. Martin Wenderoth

IV. Physikalisches Institut, Georg-August-Universität Göttingen

Date of the oral examiniation: 29.11.2021 


\section{Contents}

$\begin{array}{lr}\text { Preface } & 1\end{array}$

1 Introduction $\quad 3$

1.1 Atomistic models of friction . . . . . . . . . . . . . . 3

1.2 Structural lubricity and ultralow friction . . . . . . . . . 8

1.3 Colloidal ions trapped in potential's wells . . . . . . . . . . 12

1.4 Friction, temperature and velocity . . . . . . . . . . . 14

1.5 Ballistic friction . . . . . . . . . . . . . . . 20

1.6 Energy dissipation and friction . . . . . . . . . . . . . 23

1.7 Summary . . . . . . . . . . . . . . . 30

2 System setup and Results 31

2.1 Model and simulation protocols . . . . . . . . . . . . 31

2.2 Aubry transition . . . . . . . . . . . . . . . 33

2.3 Eigenmodes . . . . . . . . . . . . . . . . . 33

2.4 Periodic boundary conditions slider . . . . . . . . . . 37

2.4.1 Decay behaviors . . . . . . . . . . . . . 38

2.4.2 Coefficient of friction . . . . . . . . . . . 41

2.5 Open boundary conditions slider . . . . . . . . . . . . 47

2.5.1 Rigid edge . . . . . . . . . . . . 53

2.6 Other parameters . . . . . . . . . . . . . . 55

$\begin{array}{lll}3 & \text { Conclusions } & 61\end{array}$

$\begin{array}{ll}\text { A Supplementary information } & \mathbf{6 6}\end{array}$

A.1 MD implementation details . . . . . . . . . . . . 66

A.2 Logistic regression . . . . . . . . . . . . . . . 66

A.3 Additional figures . . . . . . . . . . . . . . . . . 67

$\begin{array}{ll}\text { B Reprints permissions } & 72\end{array}$

$\begin{array}{lr}\text { References } & 79\end{array}$ 


\section{Preface}

"Friction is the problem of how energy can be dissipated" [1]. As one of the oldest theoretical and applied science problems, friction plays a central role in a wide range of natural sciences branches. It embraces physics, chemistry, geology, biology, metallurgy, and engineering. Because of its multidisciplinary nature, the concept of friction is complex, yet it is not well understood [2-5]. Friction is categorized as a part of tribology: "the science and technology of interacting surfaces in relative motion" and its correlate subjects and applications. Tribology includes friction and lubrication, wear, and adhesion of surfaces in relative motion [6]. The value of lubricants was appreciated by ancient civilizations. Lubricants date back at least to ancient Egypt when used to reduce friction between sleds for transporting building blocks and wooden logs $[4,7]$. Wear is a continuous displacement and destruction at the interface of solid surfaces, appearing in the presence of relative motion between two contact surfaces. Adhesion is a newer view of friction; it measures the force required to separate two bodies in contact. In metallic surfaces, cold welding and forming metallic junctions are the reasons for friction between two layers $[8,9]$. When two surfaces move against each other, there is always energy conversion, usually through heat and noise. Losing energy always costs money. Jacob Rowe did one of the earliest estimations on the costs of friction in daily life in 1734 [10]. He suggested that just by lubricating the carriages' axles and removing friction from wheels, the number of horses' needed in the UK could drop by half. Since then, there have been enormous investigations about friction and wear in applications and their costs and methods to control these phenomena and reduce the costs of using machines $[4,5,10-17]$.

Friction is a non-conservative force, meaning that energy is dissipated or lost by transferring from one body to another or from one form to another form of energy [18]. The classic laws for modeling friction are continuum models. Amontons' friction laws relate friction force directly proportional to the applied load and are independent of the contact area. In Coulombs' law, the bodies' relative speed in the motion is independent of friction force. 
These laws are systematically breaking down [19-22]. Relative motions and velocities act in complex ways, and there are many exceptions from those rules, and sometimes friction changes unexpectedly after a period of steady behavior. These simple models cannot explain the unexpected behavior of friction in different situations. Explaining friction in a particular situation needs more sophisticated models that can formulate the system's responses in those situations [6, Chapter 1].

The large ratio of surface to volume causes extremely high friction and wear at the atomic scale; this phenomenon has narrowed the further minimization of small devices [23]. On the atomic scale, the continuum models can no longer predict or accurately explain the friction at that level. The primary reason is that the nanoscale interfaces cannot be seen as continuous surfaces that sliding over each other. The surfaces are now discrete, and they are atomic lattices. These atoms' behavior (motions) plays a crucial role in defining the properties of the lattice. These motions can change the interaction and adhesion of the interface. The force applied from one surface to the other may also vary with time because of these motions. Many theoretical models have been developed in order to explain sliding friction at the nanoscale. The occasional fast motion of the corresponding degrees of freedom, even at low velocity, induced by the instabilities at the atomic scale is crucial in describing friction. Progress in developing new techniques in understanding the nanoscale's material behavior shed new light on our interpretation of friction at the atomic level. The result from atomic microscopes and molecular dynamics (MD) helped us understand the dissipation of energy involved in the friction process [9, 20, 22, 24, 25].

Tribology subjects are deeply related to each other. Understanding them and their effect on the energy conversion process needs awareness about the highly non-equilibrium process happening at the atomic level [26]. Sliding friction is generated at the interfaces. Understanding these interfaces' properties is the most crucial part of modeling friction and, finally, finding the channels of losing energy, dissipation, or transformation to other forms of energy or other bodies [3, 4]. Sliding friction on "dry" ${ }^{1}$ solid surfaces at the nanoscale is the subject of this thesis. After reviewing some previous studies on dry friction, a two-dimensional bead-spring model will be set up for this work. Then, by employing molecular dynamics simulations, the energy dissipation through the internal degrees of freedom of the sliding object will be discussed. Also, the effect of structural defects and interaction anharmonicity on sliding friction will be investigated.

\footnotetext{
${ }^{1}$ Sliding without lubrication.
} 


\title{
Chapter 1
}

\section{Introduction}

\begin{abstract}
The chapter presents and reviews the definition of "dry" friction at the nanoscale. In this introduction, the most dominant friction models like Tomlinson-Prandtl, Frenkel-Kontorova, and some of their extensions will be discussed. This chapter also introduces the critical phenomena, such as superlubricity and thermolubricity, which appear at this scale and how velocity and temperature affect them. It also brings examples of previous studies which observed and explained these features. The system's properties, like superstructure lattices (i.e., Moiré pattern), will be reviewed. Furthermore, the mechanism of energy dissipation in the friction process will be discussed in more detail.
\end{abstract}

\subsection{Atomistic models of friction}

Bowden-Tabor model. In Bowden-Tabor's model, the contact area comprises many small asperities, making the entire area in contact much smaller than an appeared one. In this model, friction is proportional to effective shear strength and the real contact area. Many studies in this model consider the single asperities as smooth flat surfaces [20,27]. The number of these asperity areas $\left(A_{a s p}\right)$ can be verified with the applied load $(L)$ on the interface. Some latter models based on Bowden-Tabor's model again apply the continuum mechanics on each asperity. In the Herz model, this area increases with increasing load $\left(A_{a s p} \propto L^{2 / 3}\right)$. Maugis-Dugdale theory shows a linear dependency between increasing load and asperities area $\left(A_{\text {asp }} \propto L\right)$ $[20,21,27]$. The authors of Ref. [20] showed that the interface's real contact area has a linear relation with the number of atoms that chemically interact over the contact area; thus, the linearity relation between friction and interface area can be expanded to the nanoscale In another article, J. Gao et 
al. [21] argued the importance of the tribology's conversation over what has been called "real," "apparent," or "projected" interface areas in modeling friction. They said that these quantities are nonfundamental, whether at the nano-, micro-, or macroscale. The apparent area is acceptable only when shear/slip occurs at a flat molecular interface. Nevertheless, what they found is that the actual interface area can act as a scaling parameter for characterizing the other basic parameters: "the number density of atoms, molecules, or bonds involved in an adhesive or frictional interaction".

Prandtl-Tomlinson model. A point mass, $m$, is supported by a spring, with effective constant $K$, facing a one-dimensional (1D) periodic sinusoidal potential in Prandtl-Tomlinson (PT) models. The relative velocity of the mass and the potential is $v$ (Fig. 1.1).

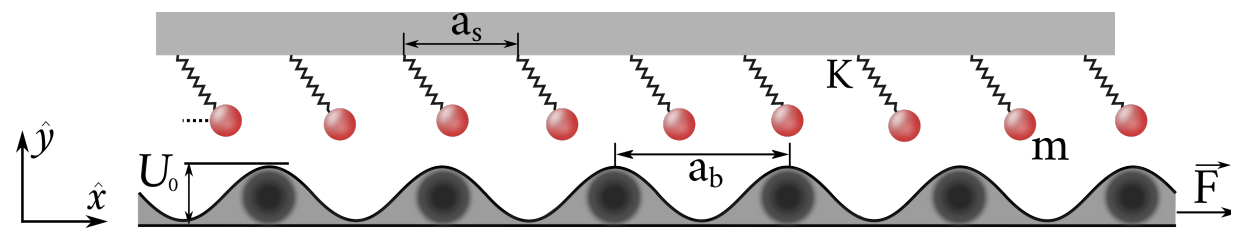

Figure 1.1: Schematic view of the Prandtl-Tomlinson model. A potential substrate, dragged with force $F$, is acting on the independent particles. The potential has an amplitude of $U_{0}$ and periodicity of $a_{b}$.

Thus, each mass experiences a total potential equal to the sum of its interaction with the substrate and the potential of the supporting spring during sliding:

$$
U(x, t)=U_{0} \cos \left(\frac{2 \pi}{a_{b}} x\right)+\frac{K}{2}(x-v t)^{2},
$$

$U_{0}$ is the amplitude and $a_{b}$ is periodicity of potential substrate. The PT model's critical parameter is the $\eta$ :

$$
\eta=4 \pi^{2} \frac{U_{0}}{K a_{b}^{2}}
$$

$\eta$ is a dimensionless parameter representing the ratio of stiffness of masssubstrate potential and the pulling spring, so called surface corrugation ${ }^{1}$. The mass smoothly slides when $\eta<1$, and its movement is a discontinuous transition known as "stick-slip" motion when $\eta>1$, and transition from

\footnotetext{
1 "Surface's corrugation or how much the binding energy to a surface varies as an atom moves from one position to another along a surface" [28].
} 
smooth sliding to the slip-stick regime happen when it is equal to one. There is no description for energy dissipation in the PT model [25, 29-32].

The PT model was first proposed to explain plastic deformation and deformation creep [33]; its primary application was found in nanotribology. The model's extension to explain friction on the two dimensional (2D) structure, the origin of friction-velocity dependency and the coupling between lateral motion and normal bring this model close to the Frenkel-Kontorova (FK) model $[25,31]$.

Frenkel-Kontorova model. In the FK model, instead of a particle or independent particles, a chain of point-mass particles coupled to their neighbors is subjected to a periodic potential [34]. A schematic view of the model is shown in Fig. 1.2.

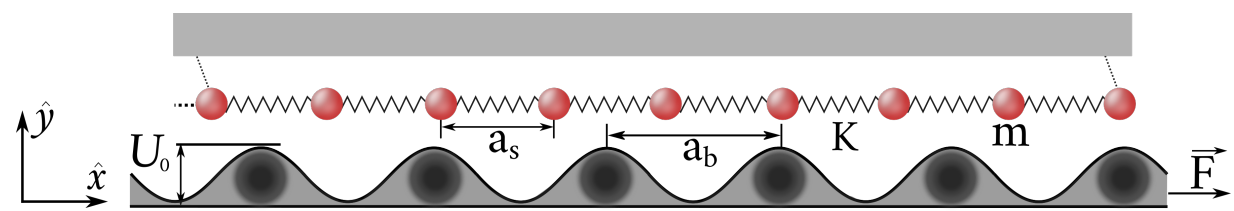

Figure 1.2: Schematic view of the Frenkel-Kontorova model: A chain of interacting particles coupled to their nearest-neighbor with elastic coupling $K$ and rest length $a_{s}$. The chain is subjected to a potential surface with amplitude $U_{0}$ and a period of $a_{b}$.

The Hamiltonian $(\mathcal{H})$ of the FK model is:

$$
\mathcal{H}=K E+U_{\text {int }}+U_{\text {sub }},
$$

where $K E$ is the kinetic energy of the particles due to displacement, $U_{\text {int }}$ and $U_{\text {sub }}$ are potential energy of linear coupling in the chain and interaction of the chain with the potential surface, respectively; thus:

$$
\mathcal{H}=\sum_{i}\left[\frac{p_{i}^{2}}{2 m}+\frac{K}{2}\left(x_{i+1}-x_{i}-a_{s}\right)^{2}+\frac{U_{0}}{2} \cos \left(\frac{2 \pi x_{i}}{a_{b}}\right)\right],
$$

which describes $i=1, \ldots, N$ harmonically coupled particles with mass $m$ subjected to the periodic potential with a period of $a_{b} . K$ is the strength between particles, and $U_{0}$ is potential amplitude [25,34]. The dimensionless parameter $\eta$ (Eq. 1.2) can be also defined for the FK model.

The FK model is essentially discrete and not integrable, but it reduces to the Sine-Gordon (SG) equation, which is precisely integrable in the continuum 
approximation. By defining $x_{n}=n a_{s}+u_{n}$ and $\left|u_{n}\right| \ll a_{s}$ the SG equation in its canonical form is defined as [34, Chapter 1]:

$$
\frac{\partial^{2} u}{\partial t^{2}}-\frac{\partial^{2} u}{\partial x^{2}}+\sin u=0
$$

The SG equation is a nonlinear model with three excitations: phonons, topological solitons, and dynamical solitons (breathers) ${ }^{2}$ [34]. The topological solitons which are better known as kinks, are what govern the tribilogical system. Kinks appear due to an inherent degeneracy of the system ground state, basically because of the "incommensurability" of the chain and substrate [35]. When the number of atoms in the chain is one more than the number of wells in the potential, we have a kink, and when one less, we have an antikink. The smallest activation energy needed for moving them is Peierls-Nabarro barriers ${ }^{3}$, which are always smaller than the amplitude $U_{0}$; thus, kinks and antikinks travel through chains easier than atoms. The motion of the kinks in the chain gives a mechanism for the particle's transportation. When the number of particles in the chain and the number of potential wells in the surface is the same, the system is commensurate. In a commensurate interface at the zero-temperature the first step to begin the sliding is to generate a kink-antikink pair. Based on the FK model, higher kink-antikink concentration leads to higher mobility [25, 34, 36, 37].

Many studies have been carried out on friction based on the FK model and kinks' behavior to understand interface motion during sliding [38-61].

Frenkel-Kontorova-Tomlinson model. Weiss and Elmer [39, 40] combined the FK and the PT models. In their Frenkel-Kontorova-Tomlinson (FKT) model, a chain of harmonically coupled particles is now coupled to a sliding body subjected to a potential surface (Fig. 1.3). Each particle in the chain is connected to the upper body by a harmonic spring.

The FKT model has higher degrees of freedom than the simple FK model. Still, particles can only move parallel to the sliding direction, i.e., it is an entirely 1D model. The potential of the FKT model in dimensionless units is [39]:

$$
\begin{aligned}
& \mathcal{H}\left(x_{1}, \cdots, x_{N}, x_{s}\right)= \\
& \quad \sum_{i=1}^{N}\left[\frac{K_{1}}{2}\left(\xi_{i}-\xi_{i-1}\right)^{2}+\frac{K_{2}}{2} \xi_{i}^{2}+\frac{b}{2 \pi} \cos \left(2 \pi\left(x_{s}-a_{s} i+\xi_{i}\right)\right)\right]-F x_{s},
\end{aligned}
$$

\footnotetext{
${ }^{2}$ The original German words for kinks and breathers were translatorische and oszillatorische Eigenbewegungen.

${ }^{3}$ Minima of the background periodic potential [34].
} 


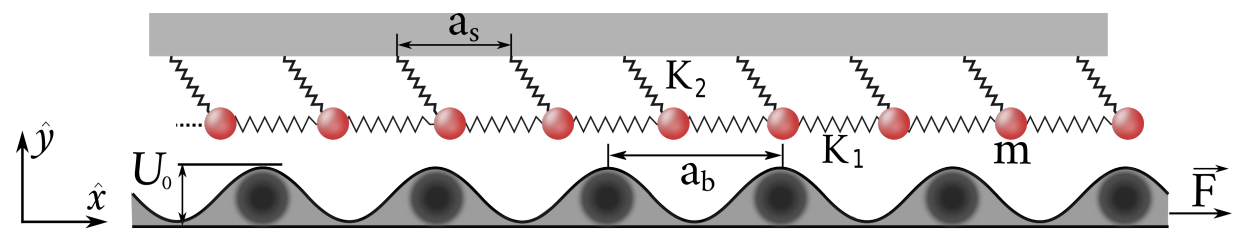

Figure 1.3: Schematic view of the Frenkel-Kontorova-Tomlinson model. Chain of particles coupled to their nearest neighbors and a sliding body with harmonic springs, $K_{1}$ and $K_{2}$, respectively. The chain is subjected to a potential surface with amplitude $U_{0}$ and a period of $a_{b}$.

where $x_{s}$ is the relative position of the upper body to the potential surface and $\xi_{i}$ is the position of particle $i$ relative to the upper body. This model predicts two main channels for energy dissipation: a velocity-related damping term and a "multi-phonon" decay process. A two-dimensional (2D) version of the FKT model was studied in Ref. [62]. This model shows the direction of motion, and also misfit angle between the surfaces can strongly affect friction in the interface.

The effects of long-range interaction and anharmonicity of the chain are discussed in the Refs. [41-45]. A. Milchev et al. modified the FK model by changing the interatomic potential from harmonic to anharmonic [41]. This modification has consequences such as the appearance of supersonic waves, breaking the kink-antikink symmetry, chain rupture or changing the model's ground state, and even changing the kinks' interaction [42]. For a low anharmonicity, the kinks are subsonic, and their velocity is independent of the applied forces. At high anharmonicity, kinks are supersonic, radiate phonons, and become unstable $[43,44]$. These radiating phonons help explain the mechanism of dissipation.

Even though the FK model simplifies atomic interactions in a crystal, its generalization to two dimension contains fundamental physics to capture the system's qualitative behavior [46]. J. Rörder et al. [47] introduced a multichain version of the FK model. In their model, two harmonic chains with another spatial degree of freedom in the perpendicular direction $(\hat{y}$-direction) are driven with steady velocity over a potential surface. J.E. Hammerberg [48] applied this model to the study of dry friction in a copper interface in MD simulations. Braun et al. [49] and Christiansen et al. [50] extend the 1D FK model to a 2D model by considering a zig-zag chain of particles coupled to their first- and second neighbors (i.e., a 2D lattice). This model embraces both transverse and longitudinal degrees of freedom [34, Chapter 11]. 
2D FK models in the continuum limit are not integrable, and even in the ground-state, these models get very complicated [51]. The dynamics of the 2D FK model is mainly studied in the dislocation theory. These dislocations are comparable with kinks in the 1D FK model. These dislocations may correspond to spatially localized excitations recognized as crowdions or the linear dislocations named domain walls ${ }^{4}[46,51,52]$. The dislocations' transportation is responsible for the traffic-jam situation in sliding a 2D layer on top of a potential surface $[51,53]$. The thermal fluctuations make a small number of particles come out of the potential wells, accelerate, moved forward, and leave an empty region behind them until they reach the front particles and jam together. Authors of Ref. [52] in the 2D FK model framework demonstrate that kinks also appear due to the developing instability of phonon modes near the dislocation axis.

\subsection{Structural lubricity and ultralow friction}

Aubry transition. The periodicity of the two competing surfaces is the prevailing characteristic of the FK model. This feature could illustrate a distinguished behavior of the configurational properties of the particles. Aubry et al. $[64,65]$ defined a crucial behavior at this transition of the breaking of anlyticity in the FK model. The chain can remain incommensurate with the periodic potential when the average distance between particles is kept fixed [66]. If particles can exist close to the maximum of the potential energy, $U_{0}$, we have unpinned ${ }^{5}$ and mobile kinks; otherwise, they are pinned. The transition between these two states, which is connected to the system's incommensurability, is known as the Aubry transition. Basically, Aubry transition corresponds to the point where the particles' positions go through a discontinuous change (non-adiabatically) [1, 67]. For this transition, there is a critical strength for springs, $K_{c}$, which defines the sliding of the lattice for a fixed substrate potential $U_{0}$. If $K<K_{c}$, atoms are pinned, and if $K>K_{c}$, kinks are mobile, and initial sliding has low energy dissipation, i.e., "superlubricity" [1, 25, 64, 67-73]. Y. Braiman et al. [66] showed that for a finite FK chain, there are three scaling parameters close to the symmetry-breaking transition. First is the phonon spectrum gap, which is the positive square root of the smallest eigenvalue of the chain. The second one is the disorder

\footnotetext{
${ }^{4}$ In other studies, the boundaries between the commensurate phases are defined as topological defects. For the 1D systems, these are described by solitons, and in twodimension, the term 'misfit dislocation' is used (for example see Ref. [63]).

${ }^{5}$ Depinning is the loss of a one-to-one relationship between the atoms of two surfaces as the surfaces move relevant to each other.
} 
parameter, which they defined as the minimum distance of any particle from the top of the potential well. Moreover, the reflection-symmetry parameter follows the breaking of the symmetry of a chain with changing $\eta$, Eq. (1.2)).

Sliding is in the superlubric regime when two hard incommensurate surfaces can slide on each other with no stick-slip motion. The friction of interfaces in this regime reduces dramatically, but it does not mean that friction would be zero. Only one of the main channels of dissipating energy originating from the instability of low-speed stick-slip motion will disappear $[25,68-70]$.

Moiré pattern. When two periodic structures overlap, they form a geometric distribution of dark and bright areas in the superposition, known as the moiré pattern. The pattern exhibits brighter domains where the structures' elements fall on top of each other ${ }^{6}$, and the darker regions between domains appear when the elements fill the spaces The dark domains create a network (i.e., lattice) which is known as domain walls. The lattice parameter and orientation of the domain structure are defined solely by the top layer's mismatch and rotation relative to the substrate. A high-symmetry rotation (based on moiré definitions) of the domain structure, related to either the top layer or the substrate lattice, matches to a local minimum of the interfacial energy. As a reaction to the adsorbate rotation angle, the system's high-symmetry state usually corresponds to an extremum of its energy. The smallest displacement, variations, or distortions can cause extreme changes in the pattern; thus, the moiré pattern has found many physical applications [57, 74-81]. 2D materials, especially graphene, hexagonal $\mathrm{BN}$, and $\mathrm{MoS}_{2}$, show remarkable tribological properties. These materials have the lowest friction coefficients in the regime of superlubricity. [82]. In some particular crystalline alignment, the friction of these materials reduced significantly, which is referred to as structural superlubricity [83]. E. Koren et al. [84] developed a method to explain the sliding force between rigid graphene twisted layers by looking at the nonlocal system breaking in the moiré pattern. They showed that the sliding force for circular graphene flake on infinite graphene substrate is governed by a rim area embracing incomplete moiré tiles.

C.R. Woods et al. [63] explained the change of behavior of a graphene monolayer on the top of a hexagonal boron nitride (hBN) substrate by using the definition of the commensurate-incommensurate transition in terms of the moiré pattern. The moiré hexagonal pattern of graphene and hBN crystal happen because of a mismatch and rotation angle of the lattices. The system

\footnotetext{
${ }^{6}$ It also could happen where the two lattices are nearly in phase [74].
} 
is in a commensurate state when the rotation angle between two lattices is small or, in terms of moiré, there is a large period of the pattern. When the angle increases, the period of pattern decreases, and lattices form an incommensurate state. It also has been shown, the reason for the superlubricity of graphite is because of the incommensurability between graphite layers. When a graphite sample rotated on the substrate because of the mismatch between the lattices, average friction exhibited ultralow values for a wide range of the angles [85]. An MD simulation study for graphene/graphene and graphene/hBN systems also exposed the moiré patterns. The results indicated that the commensurate of superlubricity is due to the progressive appearance of moiré patterns. In this study, a flake of graphene was dragged over graphene or hBN substrates. The static and kinetic friction in both systems shows dependencies on the contact size [86].

The moiré pattern also appeared when a graphene nanoflake was subjected to a graphene sheet. Despite the fact that the flake can register in a commensurate configuration with the graphene sheet, the flake demonstrates ultralow friction at low temperatures $(5 \mathrm{~K})$. The scanning tunneling microscopy images illustrated thermal fluctuations which could cause the transition from the superlubric state to the commensurate registry. When the flake runs out of the registry, it slides until it locks into another commensurate stacking with the sheet (Fig. 1.4). Their results also stated that the two states' interval in low temperature is longer than the higher one $(T=77 \mathrm{~K})[87,88]$.

A.S. de Wijn et al. [89], in a simulation study, also showed this friction dependency on temperature for flake-sheet systems. They revealed that friction for a rigid flake was also dependent on the size of the flake, loads, and scanning velocity. An examination of a series of experiments and simulations on graphite flake had also shown the flake spends most time in the locked stage. The study also disclosed that the lifetime of the ultralow friction might depend on the tip's structure and the density of defects in the interface [70]. In another investigation, they suggested the interface could exhibit stable superlubric sliding in the presence of graphene flakes [90]. The leading cause for this ultralow friction is that the sidelong forces in incommensurate (non-matching) stiff solids cancel regularly and stochastically between disordered surfaces. For these reasons, the surfaces are treated as rigid-body interfaces. If this hypothesis breaks down, there is a possibility that the system loses its structural lubricity or, in Aubry's word for the possibility of the breaking of analyticity [23, 69]. Even though the robustness of the structural lubricity in systems such as graphene/hBN has been observed experimentally [82, 91, 92], some other studies consider the system's aspects that could break 
this ultralow friction regime. D. Dietzel et al. [93] looked at the effects of the chemical bonds and contact-size on structural lubricity. Their experimental and $a b$ initio study revealed how these features could limit the structural lubricity and how the interface interaction overcame the incommensurability and determined the friction scenario.

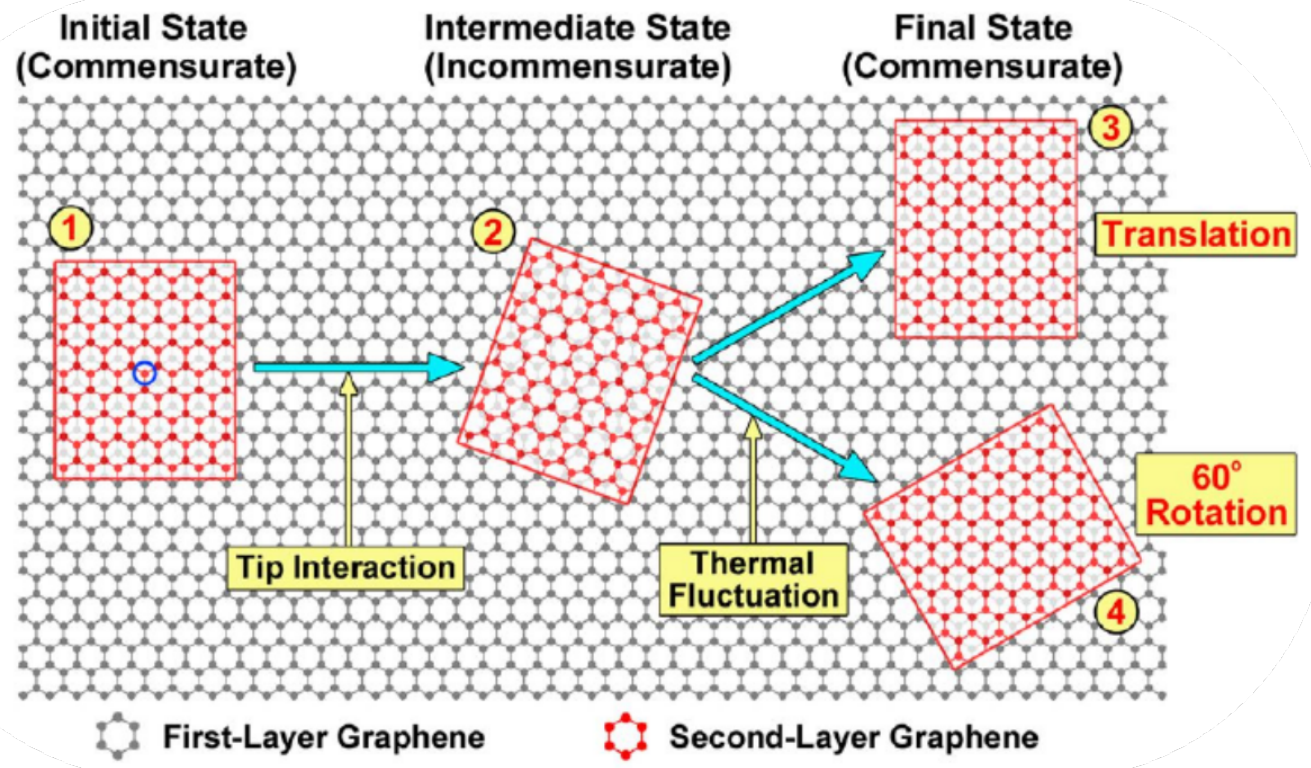

Figure 1.4: Schematic view of a graphene flake on the top of the graphene sheet. Graphene was set into motion by the tip of scanning microscopy, and then it was left free to move. Flake motion was fluctuating between commensurate and incommensurate stacking with an underlayer. In the incommensurate state, the flake slid until stuck into another commensurate state. Reprinted (adapted) with permission from ([87]). Copyright (2013) American Chemical Society (App. B).

Overall, other issues also challenge the use of superlubricity in devices. To mention a few, besides the matters which have already been discussed, surface roughness, defects, and contaminants have huge effects. Edge effects also can pin the interface. Edge pinning effects are usually responsible for the residual friction and may become dominant with growing contact size [94]. C. Qu et al. [95] developed an experimental method to decouple the edge contribution of friction from the contact area for a graphite/graphite interface. 


\subsection{Colloidal ions trapped in potential's wells}

Various methods have been presented to achieve ultralow friction at the nanoscale. Theoretically, it is possible to explain these methods by studying thermally activated masses dragged over a potential surface, like the PT or FK models [96]. T. Bohlein et al. [97] applied the 2D FK model to an experimental study. For the sliding layer, they used highly charged polystyrene spheres and suspended them in water. The spheres were ordered in a triangular lattice at the bottom of a sample cell. For the potential surface, they used a laser beam to create a periodic triangular lattice. The study considered both commensurate and incommensurate interfaces. Their result demonstrated that this "colloidal" crystal's friction properties only depend on the kinks' excitation number and density and provided direct observation of the 2D misfit's role. Their results agreed with Aubry's transition and showed the incommensurate interface's superlubric regimes [98]. E. Tosatti and coworkers [99] studied this experiment employing MD. Their results unraveled the subtle depinning mechanisms at play and showed the relevance of such experiments to friction on the atomic level.

D. Mandelli et al. [100] studied the effect of misalignment angle among a colloid monolayer and potential surfaces using MD simulations (Fig. 1.5).
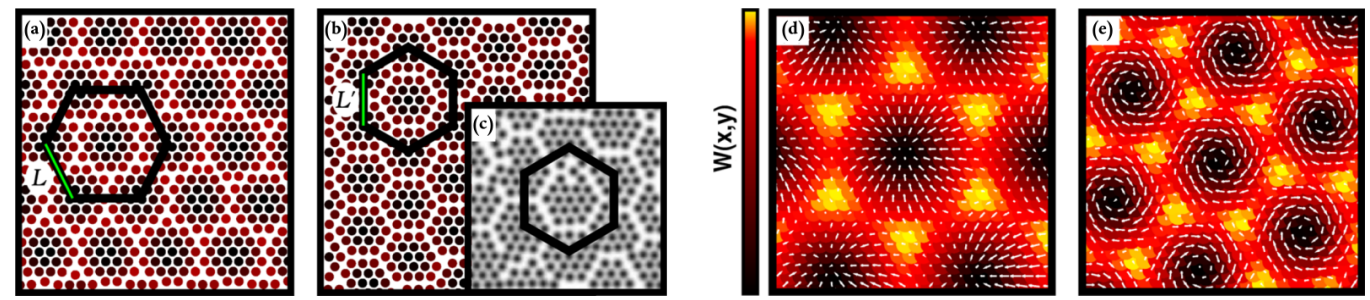

Figure 1.5: The moiré superlattice structure of a colloidal monolayer on top of optical lattice adapted from Ref. [100]. (a) shows the artificially unrotated colloidal layer with the spacing value of $L$ for the superlattice. (b) indicates the optimal misalignment angle equal to $7^{\circ}$ rotation. With this rotation, the spacing is decreased to value $L^{\prime}$. The superlattice spacing depends on the ratio of layer's lattice constants and the rotation angle. (c) shows an experimental observation of moiré pattern [which was adopted from [101]]. (d) and (e) demonstrate the moiré pattern for unrotated and rotated monolayer, respectively. The dark area of the color-coding represents the minima of the potential corrugation. The dots mark particles, and the white arrows in (d) and (e) indicate their magnified displacements. Reprinted figures with permission from E. Tosatti (M. Mandelli et al. [100]), Physical Review Letter 114, 108302, Copyright (2015) by the American Physical Society (App. B) 
They showed, in complete equilibrium, a small misalignment angle led to developing incommensurate colloidal islands. Also, while sliding, the dynamic friction increases by misalignment of angular orientation. The structural alignment changes appear in the moiré pattern, which highly amplifies the misalignment angle even if it is small. They also noted that in an incommensurate system of colloidal on laser potential, a sharp superlubric-pinned transition as a function of corrugation strength can occur. Furthermore, spontaneous rotation alters the transition point [102]. This transition remains well defined even at higher temperatures. These results indicated that the transition is highly structural and can be followed by looking at the moiré superlattices [103].

V. Vuletić and his coworkers invented an approach to trap ion chains in 1D periodic potentials set up from an optical cavity. This approach allows controlling cavity-ion strength and offers a rich model for studying the internal atomic, phononic, and photonic degrees of freedom [104]. They used the same approach and showed that stick-slip behavior of the system could be tuned from ultralow to maximum value via an arrangement of the ions relative to the substrate (potential surface). The ion continuously cooled down by laser to remove the heat generated from friction ${ }^{7}$. The light which the ion scattered was proportional to the optical-lattice potential energy. The model was applied to study the ion and substrate corrugation, which caused the interplay between structural lubricity and thermolubricity. It revealed that superlubricity could be obtained by a structural mismatch between the ion chain and the optical lattice [105-107]. Employing this model allowed them to observe a finite version of the Aubry transition. They observed superlubricity breaking by a transition from ultralow friction to the stick-slip regime increased with the potential depth [108, 109].

Another experimental and numerical study on colloidal clusters showed that for the driven cluster, orientational and directional locking indeed coincide with their geometrical moiré superstructure. Furthermore, they found that these locking behaviors on an atomic surface can be tuned via an external load. Finally, this directional locking illustrated stair-like plateaus in the migration angle. Also, their results stated that this moiré pattern gives rise to a low-energy corridor for the cluster to follow [110, 111]. They determined that these lockings could occur for any interface that contains periodic or quasi-periodic structures [112]. A 2D version of trapped ions in potential surface is illustrated in Fig. 1.6.

\footnotetext{
${ }^{7}$ Analogous to a thermostat in MD.
} 


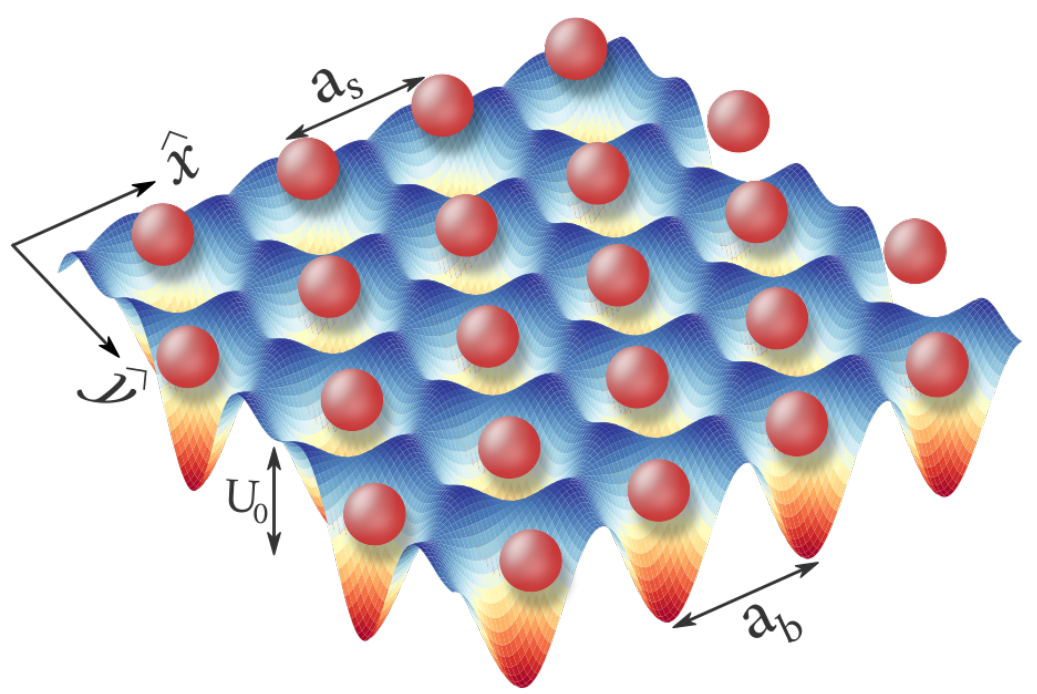

Figure 1.6: Schematic view of ions trapped in a typical potential surface with a depth of $U_{0}$. This model has been studied by many groups [113-115]. In a study by T. Brazda et al. [115], they suspended micron-sized polystyrene particles (colloidal) atop a repulsive screened Coulomb interaction. The colloidal particles form a 2D hexagonal lattice with lattice constant $a_{s}$. The periodic potential surface was created by a laser beam and had a lattice constant $a_{b}$. $U_{0}$ could be tuned by laser intensity, and the mismatch between two lattices (the incommensurability) could be adjusted by the intersection angle of the laser beams. Their approach provided a versatile method to study the superlubricity in 2D contact. Unlike the 1D model, where the Aubry transition is continuous, they observed a first-order transition at finite temperature for this $2 \mathrm{D}$ system. This phenomenon was leading to a coexistence regime of pinned and unpinned areas.

\subsection{Friction, temperature and velocity}

Temperature. In the PT model, the temperature usually affects friction by suppressing the influence of the potential depth. This effect is highly dependent on the relative velocity and also the normal load at the interface. Thermal effects can be seen in the stick-slip regime. With help from thermal activation, atoms on the surface can jump quickly from the substrate's potential barrier, which helped the interface's relative motion to last longer. Also, depending on the temperatures, the number of jumps forward and backward would be different; the temperature also alters other properties such as dragging velocity. Maruyama's simplified model [116] derived a characteristic power-law for Au cluster's stick-slip dynamics on a graphite 
substrate. The temperature caused a change in the expected behavior of the cluster on the surface. Friction between single crystal molybdenum disulfide and a silicon nitride tip showed transition from athermal and thermal friction with varying temperature from 100 to $500 \mathrm{~K}$. This study by X. Zhao et al. [117] demonstrated that describing the tip-surface with a single-1D contact model is an oversimplification, and the interface is intrinsically 2D. By considering this, they suggested that the temperature-independent friction for temperatures below $220 \mathrm{~K}$ was due to onset interfacial wear. Also, for $T>220 \mathrm{~K}$, dislocation mobility is the reason for the thermal friction. This sharp transition has also been seen experimentally for different interfaces at different temperatures. A. Schirmeisen et al. [118] reported a peak in friction with changing the temperature at $100 \mathrm{~K}$ for Si tip on Si substrate.

The friction exhibits unexpected behavior and dependency on temperature. Increasing temperatures can lower the lateral forces needed to initiate sliding and decreases the friction, and simultaneously it can reduce the slip length and increase the friction. As a result of the interplay between these two dependencies, a friction-temperatures graph can show peaks and/or plateaus (Fig. 1.7). Even though this relationship is easily altered in the presence of defects in the interface [119].

I. Barel et al. [120] simulated the tip-surface interaction with a multicontact model. The contact area was simulated using harmonic springs [121], which mimics the multi-bound forming in a virtual world interface. The temperature can manipulate the forming and rupturing bonds between the counterpart surfaces. In their model, friction depends on four characteristic frequencies: the rates of the attachment, formation, unbinding, and a characteristic rate of the pulling force relaxation. Another study also showed that the PT model's accuracy relies on parameters that describe the shape of the corrugation potential. The potential corrugation in the PT framework may result in unrealistic friction prediction [122]. J. Hassanian et al. [123] used analytical theory and simulations to investigate the role of thermal variations on the frictional response of a driven colloidal monolayer. The layer had a high elastic modulus (stiff layer) subjected to a commensurate substrate. This strong interaction leads to a sliding motion consisting of a long period of virtual a state with no motion or movement that is disrupted by the creation of small nuclei of particles that have emerged from their corresponding substrate wells, called "hopping waves". Their study predicted a transition from a sequence of discontinuous hop moves to a thermal sliding regime in which the monolayer slides in uninterrupted continuous motion. 

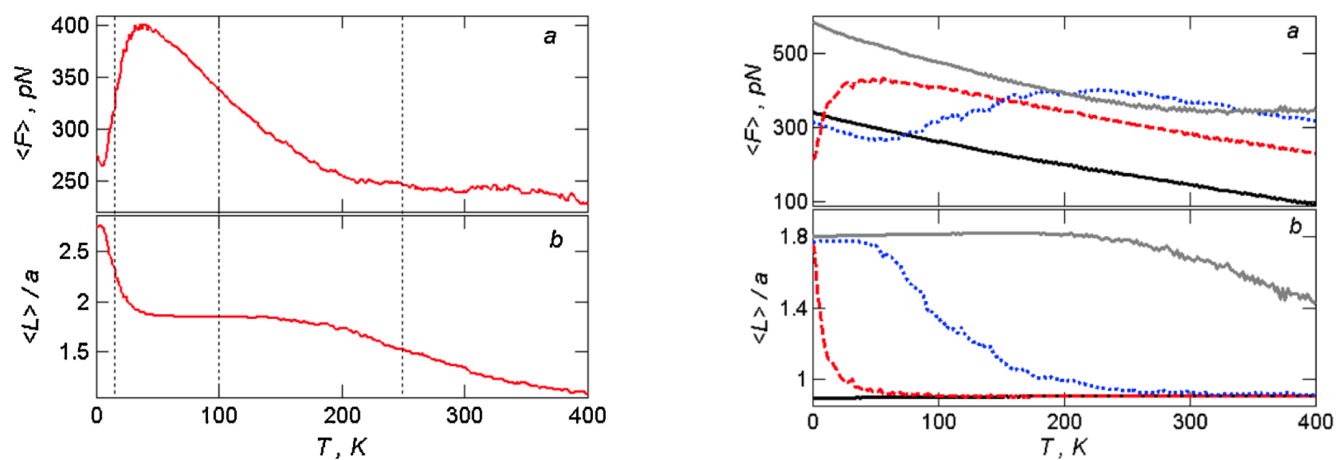

Figure 1.7: The results of temperature-induced enhancement on friction for a tip dragged over a potential surface, with surface corrugation (amplitude of the surface potential) of $U_{0}$. Left side figure: It shows the effect of temperature on the mean friction force, $\langle F\rangle$, in panel (a), and normalized mean slip length, $\langle L>/ a$, in panel (b), in which $a$ is the lattice constant. Time average friction force in panel (a) shows a sharp increase with $T$ and a gradual decrease of friction with further temperature rise; panel (b) illustrates the time average slip length. After a sharp decrease, it exhibits a plateau afterward and then decreased $\left(U_{0}=0.26 \mathrm{eV}\right)$. Right side figure: the results for four different potential amplitudes, $U_{0}=0.26 \mathrm{eV}$ (black solid curve), $U_{0}=0.33 \mathrm{eV}$ (dashed red curve) and, $U_{0}=0.39 \mathrm{eV}$ (dotted blue curve), and $U_{0}=0.51 \mathrm{eV}$ (grey solid curve). Reprinted figures with permission from M. Urbakh (Z. Tshiprut et al. [119]), Physical Review Letter 102, 136102, Copyright (2009) by the American Physical Society (App. B).

Thermolubricity. As mentioned before, thermal excitation can help the interface overcome the potential energy that acts against sliding. This assistance of thermalization may lead to ultralow friction, even if the contacts are not in the superlubric regime. Such frictional response is called thermolubric regime [29, 124, 125]. Krylov's thermolubric model is based on reversible activated jumps between potential energy wells of the interaction potential [126]. They kept the tip's velocity constant and other variables of the system fixed. In that condition, the model predicts a decrease in friction with increasing temperature [127]. Another study showed that the ultralow friction regime between a $\mathrm{NaCl}$ tip and a highly oriented pyrolytic graphite is a function of the applied load, temperature, and perpendicular actuation $[128,129]$.

Velocity. The studies on the relationship between externally imposed sliding velocity and friction revealed a logarithmic relation [118, 130-132]. Friction dependency on the velocity at the nanoscale has a complex nature. Many 
studies have shown complex behavior of friction in interface with changing the velocity $[32,131,133-154]$.

The experimental and accelerated MD study on dry friction between Pt tips and $\mathrm{Au}(111)$ in Ref. [134] pointed out that the atomic stick-slip event is thermally activated at low velocities. Also, high-velocity MD simulation leads to an athermal effect. The model in Ref. [47] for small velocities, $v \leq 0.16$ (in dimensionless units), exhibits a series of resonances corresponded to the chains' kink behavior. For the velocities in the range of $0.16 \leq v \leq 0.22$ it shows a homogeneous periodic behavior, and for higher velocity, the system displays a complex behavior. A similar trend was observed for the dependency of velocity and frictional force in the work of J.E. Hammerberg [48]. E. Gnecco et al. [135] discovered a logarithmic increase of friction with velocity between the tip and $\mathrm{NaCl}(100)$ substrate by using a thermal PT model in one dimension. They studied friction at low scanning velocity $(5 \mathrm{~nm} / \mathrm{s}<$ $v<1 \mu \mathrm{m} / \mathrm{s}$ ) at room temperature. The temperature raises the friction in the form of $F \propto \ln \left(v / v_{1}\right)$ where $v_{1}$ was taken to be $1 \mathrm{~nm} / \mathrm{s}$. Logarithmic behavior of friction was also reported in a study of friction between grafted layers on silica and a tip [136]. For higher velocities with thermal fluctuations, average friction force varies in the form of $F \propto$ const $-T^{2 / 3}|\ln (v) / T|^{2 / 3}$ in a constant temperature $T$ (also, in dimensionless units) $[130,131,133]$. J. D. McGraw et al. [137] did an experimental study on the relation of friction with velocity and the contact area between solid interfaces at a microscopic level. Their result for a contact area of hundreds of nanometers (between tip and surface) shows a linear and logarithmic relation between friction at low and high velocity, respectively. They also observed the cross-over between these two regimes experimentally. Their observations indicate that dependencies are sensitive to the nature and geometry of the tips, the number of contacts with the surface, and the experimental apparatus. Another study [139] noted that, regardless of dimensionality, when there were no thermal fluctuations, the dependency of friction with low velocity has a form of $F \propto v^{2 / 3}$. The study shows that friction-velocity dependency in the interfaces could change by thermal effects due to stochastic forces. The study was modeled by employing the PT model. They suggested the friction has a logarithmic relationship with velocity in the presence of thermal effects, i.e., $|\ln (v)|^{\alpha}$ (Fig. 1.8), in which $\alpha$ depends on $\eta$ (Eq. 1.1).

M.H. Müser [140] studied the PT model to cover the gap between frictional force dependency on low and high velocities. At low and high velocities, friction shows a linear and logarithmic relationship, respectively. He looked for a universal equation that could correctly describe kinetic friction by the 
PT model for all temperatures and velocities. This study suggested the gap can be covered by a simple friction-velocity relation $F(v) \propto \operatorname{arcsinh}\left(v / v_{c}(T)\right)$, where $v_{c}$ is a critical velocity for $1 \mathrm{D}$ system at temperature $T$ (Fig. 1.8). Although he wrote that it is difficult to extend this model for $2 \mathrm{D}$ systems.

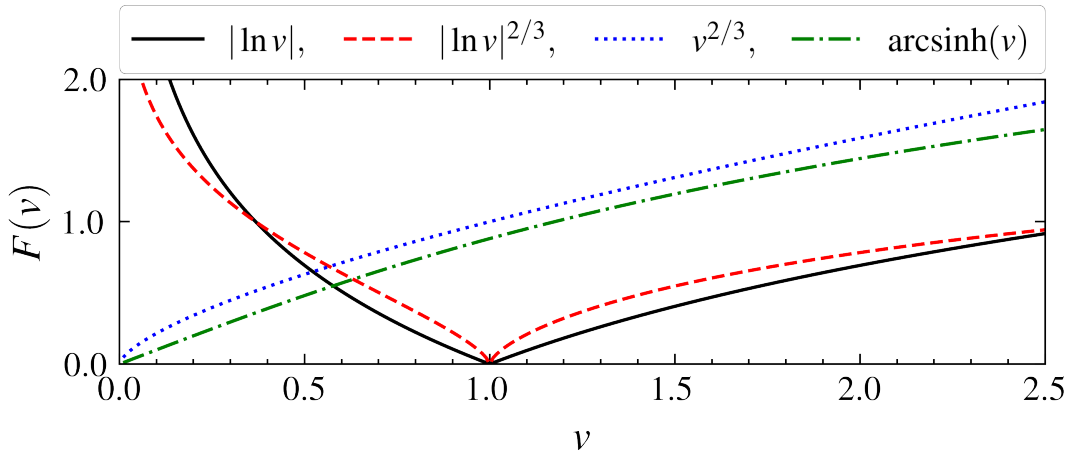

Figure 1.8: The friction-velocity relations based on the different suggestions: $F(v) \propto\left|\ln \left(v / v_{1}\right)\right|^{\alpha}$ for $\alpha=1,2 / 3$ and $v_{1}=1$ in Refs. [131, 139], $F(v) \propto v^{2 / 3}$ in Ref. [139], and $F(v) \propto \operatorname{arcsinh}\left(v / v_{c}\right)$ with $v_{c}=1$ in Ref. [140]

Another study [141] illustrated a non-monotonic relation between friction and velocity in modeling atomic microscopy experiments. It showed this relation occurred when the tip coupling to periodic potential and excitation modes of the surface is included in the model. Such a non-monotonic dependency was also reported in Ref. [142].

Friction between a tip and diamond-like carbon (DLC) surface [143], with increasing velocity, rises logarithmically, and after a critical value, friction decreases in a near logarithmic trend. The drop of center of mass velocity was due to the phase transition of DLC to a graphite-like phase. Higher velocities lead to more intense energy dissipation, and this causes the phase transformation [143-145]. Many of the theoretical models should be revisited when the materials undergo a phase transition. In graphene, the velocity-friction dependency is layer-dependent. An experimental study [147] showed that by increasing the number of layers, from monolayer to few-layer graphene, the friction force is reduced, even though, temperature fluctuations in the system could alter this dependency. A similar study [148] on molybdenum disulfide indicates that friction force increases logarithmically with increasing velocity of the tip. The substrate's number of layers did not affect this relation between velocity and friction in this case.

A study on the effects of load and velocity on friction in atomic microscopes shows that the load can change the velocity-friction dependency if the in- 
terface contains trapped ions. The applied normal load can change the influence of the ionic liquid layer between tip and surface. At low loads, the ions trapped at the contact area help sliding and the higher load, this layer is squeezed out [149].

The authors of Ref. [150] used a modified 1D PT model to explain the relation between dissipation and dynamic friction. A point mass particle dragged over a chain of harmonically coupled particles, allowing them to vibrate about their mean positions. Friction force decreases with increasing velocity, and it drops to zero when velocity exceeds the speed of sound. The substrate's phonon spectrum exhibits peaks when the slider's velocity meets the velocity of some of the phonon modes i.e., resonances. These resonances cause peaks in the velocity-friction graph as well, which indicate higher dissipation at those velocities. At low sliding velocities, deformation of the asperities is small due to low energy inserted into the system. Higher velocities which naturally lead to higher energy in the interface could deform the contact area. This deformation contributes to energy dissipation [145, 150]. D. Gangloff et al. observed four adjacent mechanisms for friction force varying with velocity. The regimes were called thermal drift, thermal activation, friction plateau, and velocity-independent regimes (Fig. 1.9). They also structurally distinguished thermolubricity from structural lubricity. The system under their investigation consisted of one or two trapped ions pulled against the periodic potential (the optical cavity), and they looked into the ion's position-dependent fluorescence. When the ion slides into the succeeding well, its fluorescence rises to maximum and drops as the ion is laser-cooled into the new potential minimum [105].

The applied force in a driving system turns into heat. In a real-world experiment, this heat transfers to the environment (heat bath). In MD simulations, this heat should be removed from the system to prevent unphysical results. This problem is handled by attaching a thermostat such as Langevin dynamics to the system. Langevin dynamics controls the temperature by approximating in the canonical ensemble (NVT) [155-159]. This procedure is done by modifying the Newtonian or Lagrangian equation of motion; thus, a viscous damping coefficient is added to the equations [160, Chapter 6]. This damping parameter will itself alter the trajectory calculated with MD simulation. One way to avoid using this damping parameter is giving initial kinetic energy (instead of applying a driving force) to the slider and thensimulate the dynamics using NVE microcanonical dynamics [151-153]. 


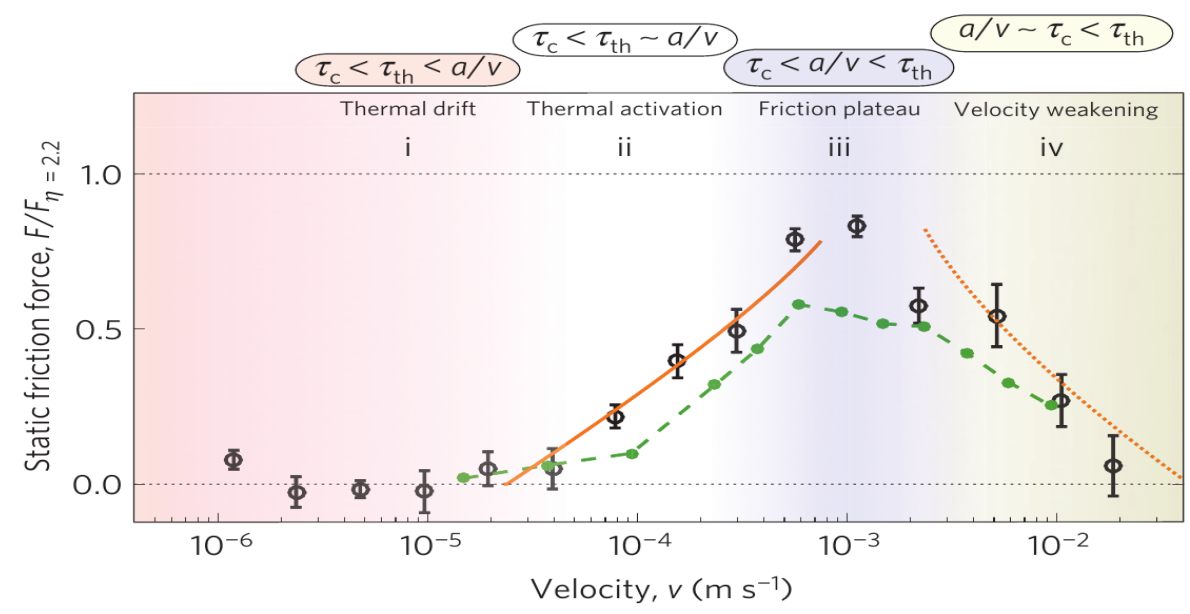

Figure 1.9: Friction for an ion, trapped in a periodic potential, as a function of the velocity. It illustrates the continuous transformation between four regimes: thermal drift, where friction is small and (roughly) velocityindependent; thermal activation, where friction rises logarithmically with velocity; and where friction is velocity-independent (the plateau part in the figure) and larger compare to the other stages; and the regime which friction decreases with velocity: velocity weakening, because of high velocity there is not much time at each slip for dissipation of energy. The three-time scales, $\tau_{t h}$ the thermal hopping time between lattice well, $a / v$ the time for the external trap to move by one lattice well, and $\tau_{c}$ the time for ion recooling. $a$ is the potential period, and $v$ is velocity. The solid orange and orange dotted lines show the expected result for logarithmic behavior for each regime, and the green dashed line shows the result from a simulation. Adapted by permission from Springer Nature: Nature Physics [105] (2015) (App. B).

\subsection{Ballistic friction}

The authors of Ref.[151] studied superlubricity of a 1D chain in the FK model. They assigned initial kinetic energy (kicking velocity) to all the particles of the chain. Since the chain slides over a periodic potential surface, strong resonances could occur if the frequency due to the initial velocity (washboard frequency) is close to a phonon mode frequency. Their results imply that the superlubricity could happen at a very low velocity in an experiment, and it can be suppressed by increasing sliding velocity and temperature.

J.W. Li et al. [152] investigated the dynamic behavior of a 1D atomic chain (slider) in a modified FK model. The substrate in their model is a chain of the same type of particles and coupled with the same bonds (springs) as the slider. Interaction between the slider and substrate is a 12-6 Lennard-Jones 
potential. The rest length of the substrate's springs is different from those in the slider, and particles can vibrate in the moving direction. This extra degree of freedom in the substrate gives rise to a nonperiodic potential. The slider was not dragged over the substrate, but all the particles were assigned the same initial velocity simultaneously. Their results show that sliding distance is not always proportional to the initial velocity, and for some velocities, the system slides shorter than what is expected. Since the slider and substrate have the same spring strength, they have the same eigenfrequency, and there was more phonon excitation. They explained the decay of the slider's center of mass velocity based on the system's phononic properties. When the atoms' vibrations are small, there is a superlubric "stage", and the second stage is a "single-mode" phonon excitation, where the interchange of internal and kinetic energies happens. Multi-phonon excitation is the last stage when kinetic energy of forward motion irreversibly transformed to internal energy. J. Norell et al. [153] studied a "straightforward" extension of the FK model in a 2D square lattice. A square lattice consists of springs, and mass-pointlike particles are kicked over an incommensurate potential landscape. They considered the scalar and vector extension of the FK models [34, Chapter 11] separately. The scalar model cannot describe the system precisely since it is composed of two decoupled 1D systems without interaction. They calculated the viscous friction parameter by fitting the decaying center of mass velocity. This parameter is a function of the kicking velocities.

Another MD study on friction showed that slip proceeds by a commensurate domains' nucleation and growth in a commensurate interface. In this work, M. Reguzzoni et al. [161] studied the onset of a Xe monolayer's slip on a $\mathrm{Cu}(111)$ substrate in thermal-activated creep under a small external lateral force. The study indicates that Xe monolayer's interatomic forces play a major role in the slip activation process. They also find that the nucleation barrier energy is linearly dependent on the interatomic strength and the corrugation of the potential surface. They also suggested that defects could be the reason for the experimental observation of sliding in the adsorbed solid monolayer of $\mathrm{Xe}$ on $\mathrm{Cu}(111)$ surfaces (even though they are commensurate) since disorder can strongly reduce the nucleation barrier energy. T. Coffey et al. [162] observed slip in commensurate $\mathrm{Xe} / \mathrm{Cu}(111)$ system having less friction compared to non-commensurate $\mathrm{Xe} / \mathrm{Ni}$ and $\mathrm{Xe} /$ graphene systems. They assumed that the friction in this study is primarily phononic friction. An analytical study on the effect of dilute concentration of particles (adsorbed molecules) trapped between two rigid surfaces also showed that friction in this situation depends on each surface corrugation and mobile molecules' interaction [163]. M. Pierno et al. [164] They explained how superlubricity appears in size large size limit of naturally incommensurate Xe islands on the 
$\mathrm{Cu}(111)$. They showed how the defects- and edge-related defects limit the superlubricity caused by structural incommensurability. They concluded that a spontaneous compression on the monolayer (caused by adhesion) transforms the interface into a commensurate phase, resulting in a sudden pinned state. R. Guerra et al. [165] studied frictional sliding of diffusive Au clusters, which were "kicked" on a graphite substrate in MD simulation. Their result exhibited two different speed-dependent behaviors for the clusters. The cluster experiences ballistic friction at high speeds, in contrast to a drift motion at low speeds. In these two regimes, the friction shows an opposite dependency on the temperature. The interplay of translation and rotation had a significant effect on controlling the crossover between the two regimes (Fig. 1.10). Furthermore, the authors investigated size effects and noted that the substrate's thermal fluctuations had a lower effect on the heavier clusters than on the smaller ones [166].

An experimental study on such a diffuse system was done to measure the frictional damping in motion of single benzene molecules thermalized on a graphite surface [167]. They found that while the observed motion was controlled by kinetic dissipation (mainly by the creation of phonons), the role of the substrate potential was insignificant.

Y. Liu et al. [168] also studied kicked flakes on graphite surfaces in further detail. They investigated the effect of the initial temperature, flake misalignment with the substrate, and its size and shape on sliding friction at high speeds. The step-like decaying of the center of mass velocity occurred for all flake sizes and shapes. Their results also confirmed the effect of misalignment angle (observable in the moiré pattern) on the incommensurability and its reduction of friction. They suggested two ways to reduce sliding friction for their system first, by using graphene nanoribbons to prevent the flake's rotation (instead of using infinite substrate), and second, to apply strain to the substrate to change the lattice constants' ratio. Both these approaches reduce the commensurability between the flake on the substrate and thereby friction. A similar study also showed the effect of the flake size and temperature on a flake's sliding behaviors over a graphite substrates employing $a b$ initio and MD [169]. The effect of annealing temperature and flake size was investigated experimentally by D. Wang et al. [170]. The study revealed that graphene flakes rotate on hBN substrate, and a moiré superlattice with a large spatial period can be achieved by annealing the system. For annealing at temperatures $>100^{\circ} \mathrm{C}$, the system showed a stable and metastable state for rotation about $0^{\circ}$ and $30^{\circ}$, respectively. Besides, they observed a critical rotation $\left(\sim 12^{\circ}\right)$ that tends to rotate toward that two states.

Another study on this subject reported that the commensurate system composed of a graphene flake on a graphene substrate could lead to a superlubric 


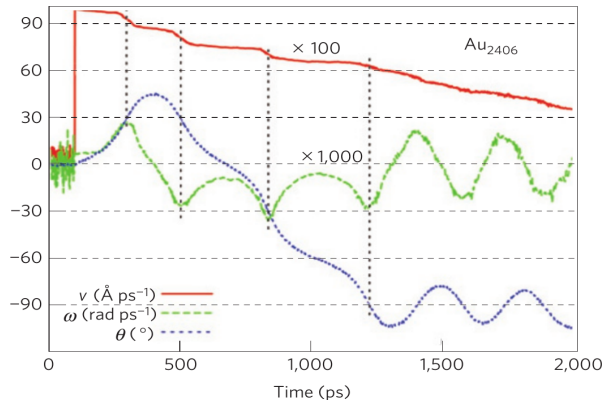

(a) Slowdown of kicked Au clusters

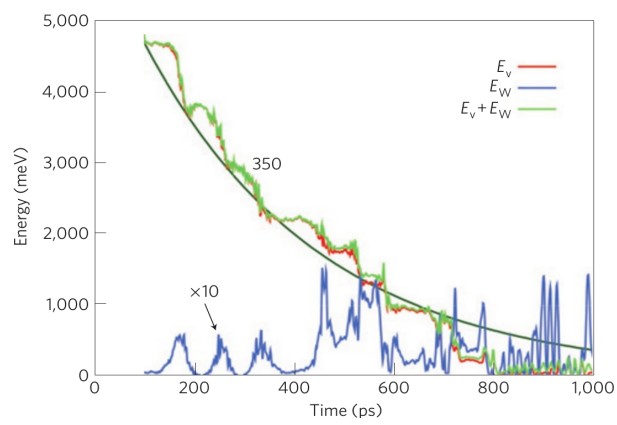

(b) Kinetic energy of Au clusters

Figure 1.10: Frictional slowdown behaviors (a) and the time decay of kinetic energy (b) for ballistic clusters. In (a) the slowdown of the center of mass (red solid line) comprising tilted plateau for the out-of-registry cluster-graphite configurations that correspond to the superlubric incommensurate friction and clear drops at unexpectedly perfect matching angles. The translationrotation exchange shows the friction force and torque are inherently coupled. (b) shows how the translational $\left(E_{v}\right.$, red), rotational $\left(E_{\omega}\right.$, blue), and total (green) kinetic energies of the kicked cluster act. The cluster was first thermalized and then got a strong side-kick with zero rotational speed. Both decay approximately in an exponential shape. Adapted by permission from Springer Nature: Nature Physics [165] (2010) (App. B).

incommensurate state and an anomalous fast diffusion of the flake in these systems. Moreover, the increase in temperature would lengthen the time spent in the incommensurate state. It showed that the flake's diffusion coefficient depends exponentially on the differences between commensurate and incommensurate states and the energy barrier for the transition of the flake between adjacent energy minima in the commensurate states [171].

\subsection{Energy dissipation and friction}

It is generally accepted that the electronic and phononic channels of energy dissipation are the main ways for converting kinetic energy during frictional sliding. Different phononic and electronic properties of materials, when their thickness is reduced to a few or one layer, may impact the frictional behavior. Thus, there has been an extensive investigation in understanding the kinetic energy dissipation to the bulk. The studies indicated that the number of layers [172] and the interlayer stiffness could change the system's frictional properties. The vibrational energy of the interface atoms transfers to the bulk 
material as propagating phonons ${ }^{8}$. For instance, in metals, electron-hole pair excitations can damp these vibrations. Still, one of the main challenges is understanding the behavior of atoms on the interfaces [83, 127, 174, 175].

The practical inaccessibility to the interface between moving bodies in many cases has made it difficult to follow the interface's tribological behavior [99]. Friction in buried interfaces can be affected by many factors, such as temperature, defects, load force, size. Each of these factors could end up establishing new pathways for energy dissipation during sliding. Many analytical methods and computational analyses have been done to understand the mechanism of these dissipations [28, 72, 73, 88, 176-200]. Dissipation of energy in friction phenomena usually happens by converting the kinetic energy into heat. When atoms in one surface get excited from atoms of the opposite surface, the energy of these vibrations (phonons) is eventually dissipated as heat (phononic friction). By definition, in classical thermodynamics, this process is irreversible. Nevertheless, if the process happens in a quasistatic way, i.e., the process is completed slower than the system's relaxation time, then the interface remains in equilibrium; thus, the process can be considered reversible. However, any instability in the system will result in unrecoverable energy dissipation $[28,127,176]$. The mechanism of energy dissipation pathways in the frictional system is highly system-dependent. Here, a few examples of the previous studies will be reviewed.

The FK and FKT models. Energy dissipation in the FK model, which considers the atoms' motion in only one of the layers, is explained by these atomic motions. In this model, the degree of incommensurability has an enormous impact on the energy dissipation during sliding. Even though the atoms in the FK model are no longer independent, they are still decoupled vertically from the upper layer. In the FKT model, atoms are vertically connected; however, one of the surfaces' atomic motion is still absent [176, 178]. L. Consoli et al. [177] studied the onset of friction of undriven harmonic chain sliding over an incommensurate periodic lattice in the FK model framework. Their model system did not contain any arbitrary damping parameters. They concluded that the onset of friction could be assigned to a unique type of dissipative parametric resonances, which results from the coupling between the center of mass velocity and the washboard frequency [28].

\footnotetext{
${ }^{8}$ This process depends on the vibrational frequency of the absorbed and maximum phonon frequency of the bulk, and it is layer-dependent [173].
} 
Alkaline chains. J.N. Glosli et al. [178] reported a study on the behavior of friction and energy dissipation in the interface of ordered monolayers of alkaline chains, using MD simulations. The interaction between atoms on chains was a smoothed LJ potential. For smoothing, they truncated the potential so that it would be zero beyond a cut-off radius $r_{c}$. The system was oriented in the $x-z$ plane. Also, the chain of atoms is assumed to be lying along the $x$-axis. Moreover, the upper layer was dragged with constant velocity in the $x$ direction. The chains of the carbon atoms were allowed to twist and bend but not stretch. A heat bath held the temperature of the layers constant. They observed two different mechanisms for dissipation of energy in the system: a discontinuous behavior arising from a sudden release of shear strength, which they called the "plucking" mechanism, and behavior from collisions of atoms with the opposite layer. In the pluck mechanics, the system's stored-energy as strain, is transformed to thermal energy, and is observed at low velocity and temperature. In a weak interaction for low $(T=20 \mathrm{~K})$ and very high $(T>100 \mathrm{~K})$ temperatures, the friction force was low, the plucking mechanism disappeared, and a thermal activation model can describe it. At low temperatures, the system is a combination of harmonic oscillators with an adiabatic separation where the translational and vibrational modes of the chains are decoupled and, it seems in these temperatures, the friction is independent of the sliding velocity. At the highest temperatures, the system vibrates so actively, the system is made to slide smoothly over each other. However, in middle temperatures $(T=80 \mathrm{~K})$, the frozen torsional modes become excited and coupled with the other modes, raising the anharmonicity and resulting in higher friction and more energy dissipation [176, 178, 198].

EDR. Z. Wang et al. [179] adopted the energy-dissipation ratio (EDR) to describe energy dissipation in a 1D PT model during stick-slip motion. In their MD study, an atom bonded to driving support by a harmonic spring and dragged over a Periodic potential. The EDR describes the relationships between the energy that dissipated enduringly in the system and the conservative reversible energy that can be reintroduced to the driving system after the slip process. Their study shows that stored energy in the stick stage does not always dissipate. They used the EDR to determine the relationship between total dissipated energy and recoverable energy in the stick stage. The EDR is dependent on intrinsic properties of the system and influencing factors such as velocity, temperature, damping factor, and it can vary from zero to a hundred percent. 
J. B. Sokolof. A further, more extensive attempt to elucidate obscurity of energy dissipation of friction was made by J.B. Sokolof [73, 180-187] and J.B. Sokolof et al. [188-193]. In some sense, these studies are a generalization of the FK model.

Glide dislocation-motion in crystalline solids results in slip. For a perfect crystal, in a slip plane, one plane of atoms slides over another [201, Chapter 1 and 3]. J.B. Sokolof modeled this motion at low speeds, considering that lattice vibrations' excitation causes dissipation in one surface by the other. Sokolof calculated the average force of kinetic friction when these planes are moving relative to each other [182]. In this model, atoms in the slip plane are harmonically connected to their first neighbors via springs in a simple tetragonal lattice. This plane is subjected to a sinusoidal potential (Fig. 1.11a). A phenomenological damping constant is assigned to each phonon mode of the slip plane through which the phonon modes' energy dissipated. Incommensurability of the slabs plays a crucial role in this model. An incommensurate-commensurate transition can introduce a domain wall (kinks) in the lattice. He found that the frictional energy dissipation is very much smaller (of the order of $10^{13}$ ) at low speed (compared to the velocity of sound in the material) when the potential energy is incommensurate with the slip plane compared to the commensurate case landscape. Also, the friction is proportional to the area of slipping and inversely proportional to the sliding speed. Sokolof solved the friction relation at higher speeds by assuming that the sliding speed is high enough that the atoms' displacement is small and do not have time to $\operatorname{relax}^{9}[28,180-183]$.

In [72], Sokolof determined the critical size for a real solid (a chain of particles) needed to cause energy dissipation. He had shown before that significant energy dissipation only occurs if the spacing between modes is smaller than the inverse phonon lifetime. Since the mode spacing increases with decreasing system size, there should be a critical size for which sliding causes dissipation. Anharmonicity, which causes the transformation of energy between modes by means of phase scattering processes (such as Umklapp), is the main reason for phonon damping. An anharmonic coupling between a harmonic oscillator and other oscillators will only exchange considerable energy if its frequency is equal to a linear combination of those oscillators' frequencies. For finite systems, this rarely happens because the modes are discrete for the finite system. To show this, Sokolof considered a Fermi-Pasta-Ulam-Tsingou (FPUT) chain fixed at one end and applied a harmonic force to the other end. In the FPUT model (a chain of a finite number of atoms), the energy is first

\footnotetext{
${ }^{9}$ This result was also observed in other studies, such as phonon energy dissipation in friction between graphene/graphene interface [198].
} 


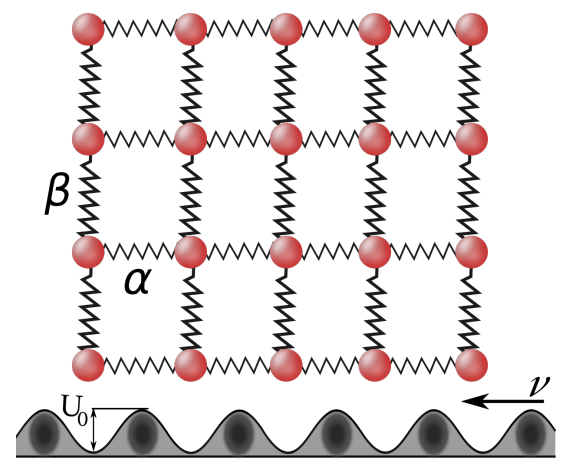

(a) Sokolof's model

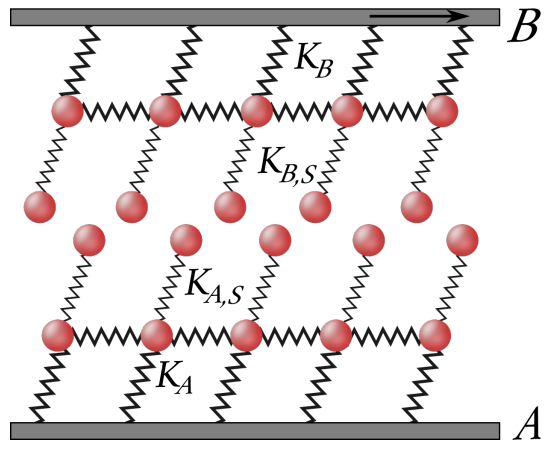

(b) Xu's model

Figure 1.11: Two different modifications of the FK model: a) A schematic of a typical Sokolof's model: A sliding crystalline lattice subjected to an incommensurate periodic potential. Atoms in the lattice connected to their first neighbors via harmonic springs in two directions. The spring's constant in the vertical direction is $\beta$, and in the parallel direction is $\alpha$. The potential slab and the upper lattice are moving in the relative velocity $v[28,182-$ 184]. b) Xu's model: Objects $\mathrm{A}$ and $\mathrm{B}$ consisted of the atoms connected via harmonic springs. The bulk atoms of each object are connected to their nearest neighbors. Surface atoms are only connected to the upper and lower atoms for objects $\mathrm{A}$ and $\mathrm{B}$, respectively. Object $\mathrm{A}$ is constant, and object $\mathrm{B}$ is moving with steady velocity. Both objects vibrate elastically with a lower frequency, and the authors named them macroscopic oscillators. Atoms at the surface can freely move, and since they vibrate the same as the phonons, they are named micro-independent oscillators [28, 200].

put into a single vibrational mode with a small amount of anharmonicity. Since the anharmonicity is small, the chain is nonergodic; hence energy is not spread between other modes of the system in time. The study showed that there is a finite size for which the friction is almost zero. This effect is due to a lack of ergodicity in the finite system [72, 73, 182, 185, 191]. Sokolof then showed that the existence of a lifetime for lattice vibration plays an essential role in determining the velocity dependence of friction [183]. J.B. Sokolof and colleagues in Ref. [188] showed for $\eta<0.5$ despite the lack of translational symmetry in the FK model, the model's structure factor displays sharp phonon peaks like a system with the symmetry. They conclude that to determine phonon damping, higher order perturbation theory should be considered. 
Phononic friction. M. Cieplak et al. [24] employed MD simulation in combination with analytic theory to model the origins of friction. For this study, they used $\mathrm{Kr}$ as adsorbents sliding on top of a rigid periodic hexagonal substrate. Their model included only one source of friction: from vibrations (phonons) within the krypton atoms as they slipped, and no energy dissipation was associated with the vibration of gold atoms in the substrate. They showed that the anharmonic term in interaction leads to a constant draining of the energy into other phonon modes. Increasing the temperature and substrate corrugation caused a rise in friction levels [28]. The MD simulation study on $\mathrm{Xe} / \mathrm{Ag}(111)$ film by M. S. Tomassone et al. [190] also indicated that the phonon excitation makes a dominant contribution to the system's friction. They calculated the slip time for the system, in a thermostat-free environment, as a function of the number of atoms, $N$, in the film (i.e., coverage). Slip time in frictional energy dissipation is the time that the slider center of mass velocity falls to $1 / e$ of its original value. The results for $60 \leq N \leq 370$ illustrated a dip of 0.0563 atom $/ \AA^{2}$ and a sharp rise after increasing the coverage. Whereas A. Liebsch et al. [202] reported that, for Xe sliding on $\mathrm{Ag}(001)$ and $\operatorname{Ag}(111)$, the electronic and phononic distribution channels are of similar importance. The importance of these channels revealed a strong dependency on coverage. Their simulation study indicated that phonon friction rises with coverage, and the electronic channels are roughly coverage-independent in overall sliding friction. J. Nakamura et al. [203, 204] showed that the friction force in a driven cluster on a periodic potential is size-dependent. In the FK model framework, they find a magic size, in which the critical force strength of depinning is reduced to a minimum amount for constant velocities [28]. Y. Mo et al. [205] quantitatively explained that the surface coverage could be the reason for lower friction observed [206] in passivated surfaces with deuterium instead of hydrogen atoms. The passivation layer's chemical stability is higher for deuterium than for hydrogen. They demonstrate that this stability eventually leads to greater surface coverage and less friction for the more massive isotope. The authors also mention that the shear strength on the absorbing layer for different coverage is what makes differences in the observed friction.

Z. Xu et al. [200] introduced a composite model of dependent oscillators to obtain a numerical solution for "phonon-based" energy dissipation in interfacial friction (Fig. 1.11b). Each surface consists of macroscopic oscillators at bulk, describing the friction behavior at the equilibrium stage, and microscopic independent oscillators at the surfaces to describe the non-equilibrium stage of the friction process. The micro oscillators also determine the dissipation process in the model. When the frequency of the forces at the surfaces 
is high, the energy is dissipated to heat irreversibly. An experimental and simulation approach was used to study gold nanocontacts sliding on graphite in the work of A. Lainé et al. [207]. In this work, increasing amplitude oscillatory shear force leads to a one-shot investigation of friction aspects. Their model uncovered phenomena that bridge the gap between initial depinning and large-speed sliding.

The phononic energy dissipation process in a system containing a single asperity or single lubricant molecule in the interface was studied by A. Buldum et al. [208]. They performed a quantitative analysis of energy dissipation in sliding friction by assuming the interface was weakly coupled. As was already noted by Sokolof, phonon lifetimes are an essential parameter in understanding friction. Another study showed that the phonon lifetime of some phonons gradually decreased with increasing dragging velocity. L. Consoli et al. [209] suggested that the translation from a floating (incommensurate state) to a pinned state is related to the appearance of Umklapp terms as a result of phonon excitation. They also noted that, for an undamped 1D FK model, the origin of friction and the appearance of Umklapp terms are the nonlinear couplings and resonant phonon excitations. However, these effects remain as two different appearances happening at separate times. The relation between the multiphonon process and friction was studied in Ref [198]. Z. Wei and colleagues considered phonon properties in graphene/graphene interface using MD simulation. They found that some phonons' lifetime, specifically the modes around the $\Gamma$ point of the first Brillouin zone, slowly decreases as the sliding velocity increases. This phonon lifetime-based model is proposed to explain the frictional force's variation as a function of the sliding velocity; in other words, under the same temperature, the higher friction force corresponds to the shorter phonon lifetime. They also predicted that the friction force increases logarithmically with the sliding velocity. Their study was an effort to demonstrate the decrease of phonon lifetime (improvement of phonon scattering strength) as means to raise the friction force. Cammarata and Polcar suggested that it is plausible to control frictional energy dissipation by controlling the phonon-phonon interaction strength. This interaction can be determined by the equilibrium properties and atomic types of the system. They studied layer sliding in transition metal dichalcogenide thin films. It was observed that it is possible to tune energy dissipation due to friction by fine-tuning the phonon coupling [199]. They found that when the bond character was more ionic, the energy dissipation reduced. Moreover, some specific atomic orbitals and their contribution to selected system vibrations significantly impact the frictional response in tribological conditions. 


\subsection{Summary}

Many factors influence friction. In many respects, friction is highly complicated and depends on an extensive spectrum of intrinsic and extrinsic circumstances. There is no theoretical framework where the frictional work could be calculated quantitatively in all cases. According to simple models (such as PT), strong interfacial interactions (corrugations) give a finite static friction force, then stick-slip motions between atoms. Friction also increases by anharmonic coupling between excited phonon modes, which initiates new passages for energy dissipation. Among other excitation modes, defects at interfaces, counterparts' density, and electron-phonon coupling in metals can also magnify friction. Intrinsically, the atomic structures and configurations of 2D materials such as thickness, structural defects, dangling bonds can significantly affect their frictional response. The commensurability of the interfaces can modify the friction by many orders of magnitude. Besides this, other structural properties of the system can alter friction in the interfaces. For example, the size of the system plays a crucial role in the sliding process. In three-dimensional systems, the extra freedom, normal to the surface, could also reduce energy dissipation. This third dimension gives degrees of freedom to atoms to escape the stick events along the sliding direction.

Temperature is critical in describing the sliding process. It influences frictional behavior in several ways. It can increase friction by activating the energy-dissipating mode. In contrast, it can assist the atoms with jumping over the barrier, thereby reducing friction. At higher temperatures, many more phonon modes can get excited, which indeed results in higher friction and more energy dissipation. Still, at a very high temperature, close to melting point and beyond, the friction gives liquid-like friction. ${ }^{10}$ instead of the solid one.

Based on the studies reviewed here, phonon and electron mechanisms both contribute to the dissipation process. Phonons' lifetimes and their interaction with electrons can define the system's frictional behaviors. Many simplifying models based on one or both of these processes have been formulated to embrace existing systems' complexity with theoretically tractable models that retain friction's fundamental aspects. Phonon models of friction are straightforward for solids. There are clear and simple geometric compositions of the component atoms, illustrating the way wear-free friction might arise at the nanoscale from the corresponding motion of atomic lattices in sliding contact $[25,28,83,96,122,132,176,210,211]$.

\footnotetext{
${ }^{10}$ Viscous friction.
} 


\title{
Chapter 2
}

\section{System setup and Results}

\begin{abstract}
The kinetic energy of a sliding object in a coherent motion eventually turns into heat, i.e. random internal motion. In this chapter, friction on motion between a 2D stiff substrate and a 2D sliding layer will be discussed. The slider is placed and kicked on the top of the sublayer ${ }^{1}$. By looking at the effects of anharmonicity, defects and initial velocity, we will study the frictional behavior of the system. This chapter will consider a slider with two different boundary conditions, namely a system with periodic boundary conditions (pbc) and a system with an open boundary conditions (obc). Thermalization begins for pbc sliders by those modes whose spatial modulation is well-matched with the incommensurate lattice. For an obc slider, the most dominant vibrational modes are rotation, torsion, or breathing-like vibration. By adjusting the mechanical properties of the slider's edge, activities of many of these modes can be managed, resulting in striking changes in how far the slider slides ${ }^{2}$.
\end{abstract}

\subsection{Model and simulation protocols}

Slider. The 2D layer is represented by $N$ classical point-like particles with mass $m$, set in a triangular lattice with an equilibrium lattice constant of $a_{b}$. The ratio between the lattice's sides is $L_{y} / L_{x}=\sqrt{3} / 2$, in which $L_{x}$ and $L_{y}$

\footnotetext{
${ }^{1}$ For this model, it is better to say that the potential surface moves against the slider and the potential energy is transferred to the slider [e.g. see Ref. [183]], but it is more convenient and common to say that the slider is kicked on top of the surface. Hence, in this chapter, we will talk about the kicked slider [e.g. see Ref. [153]].

${ }^{2}$ The main part of this section is published in Physical Review E 104, 014802, 2021, by the American Physical Society: Friction on incommensurate substrates: Role of anharmonicity and defects, S. Amiri, C. A. Volkert, and R. L. C. Vink (App. B).
} 
are the system's length in the direction in $x$ and $y$ directions. The particles interacting via the following equation:

$$
U(r)=\sum_{n=2}^{4} \epsilon \alpha_{n}\left(\frac{r}{a_{s}}-1\right)^{n},
$$

which we found by getting Taylor expansion of the the Lennard-Jones (LJ) potential $[182,184]$. In Eq. $2.1 r$ is the distance between two particles sharing a bond and $\epsilon$ (well-depth of LJ potential) sets the energy scale, and $a_{s}$ is the slider lattice constant. For the anharmonic bonds, the $\alpha_{i}$ coefficients are as follows: $\alpha_{2}=36, \alpha_{3}=-252$, and $\alpha_{4}=1113$. For harmonic bonds, $\alpha_{2}=36$, with the remaining coefficients set to zero. We emphasize that in $2 \mathrm{D}$ and $3 \mathrm{D}$ systems, this choice does not render the system completely harmonic. A small geometric anharmonicity [212] remains, due to the square-root term that is needed to compute the separation $r$. In our simulations, for the harmonic system, the energy was thus computed slightly differently as follows: Taking the perfect lattice as a reference, the potential energy of the harmonic bond between particles $i$ and $j$ is given in terms of their displacement vectors from the perfect lattice position as [213]:

$$
U(r)_{i j}=\frac{\alpha_{2}}{2}\left[\hat{a}_{b} \cdot\left(\vec{u}_{j}-\vec{u}_{i}\right)\right]^{2},
$$

which ensures a truly harmonic system, i.e. quadratic in the displacements (in the above, $\hat{a}_{b}$ is the equilibrium lattice unit vector connecting particles $i$ and $j$ ).

Substrate. The substrate, which resembles a corrugation potential, was modeled by stationary point-like obstacles in a fully periodic triangular lattice with lattice constant $a_{s}$. The number of these obstacles is $M=\operatorname{int}\left(g^{2} N\right) ; g$ is the golden ratio and equal to $(1+\sqrt{5}) / 2$ and $\operatorname{int}(x)$ is the largest integer smaller than or equal to $x$. This indicated that the substrate is denser than the slider. In this way, the lattice constant ratio $a_{s} / a_{b}$ is as close as possible to the golden ratio and ensures maximum incommensurability.

The slider was set atop the substrate so that the particles in the slider interact with the obstacles in the substrate via a soft potential with $\alpha=0.3$ and $r_{c}=a_{s} / 2$ as cut-off radius, in the form:

$$
U_{\text {soft }}(r)= \begin{cases}\alpha \epsilon\left[1+\cos \left(\frac{\pi r}{r_{c}}\right)\right] & r<r_{c} \\ 0 & \text { otherwise }\end{cases}
$$


Units. For the model we studied in this work, we used dimensionless reduced units. It is possible to define dimensionless reduced units based on the LJ potential parameters, suitable for molecular simulations [123, 140, 153, 214]. In these units, lattice constant $a$ in the equilibrium is the length units, a reference mass $\left(m^{*}\right)$ is used for mass unit of particles, temperature's is given order of $\epsilon / k_{B}$, where $k_{B}$ is the Boltzmann constant, and energy in other hand, is in units of $\epsilon$. This indicates that time is in unit $[t]=\sqrt{m^{\star} a^{2} / \epsilon} \sim 2.6$ ps presuming a slider made up of Cordon atoms $(\epsilon \approx 2.76[\mathrm{meV} /$ atom $], a \approx 3.8[\AA]$ and $\left.m^{\star} \approx 12 \mathrm{u},[215]\right)$. All the results in this work will be presented in the dimensionless reduced units (LJ units).

Simulation specification. All simulations were carried out using molecular dynamic software LAMMPS (Large-scale Atomic/Molecular Massively Parallel Simulator) [216]. A trajectory that is the particle's positions and velocities during the simulation time is the essential simulation output. From this trajectory, other quantities of concern can be calculated. The details of the implementation are given in the Appendix A.1.

\subsection{Aubry transition}

The Aubry transition in the framework FK model is the transition point where the simultaneous elimination of a sliding mode with zero-frequency happens at a crucial number of the bond's hardiness of the external potential [217]. The slider is pinned for the value bigger than the critical strength, and for the valve below that, it slides smoothly. The quantity that can reflect all particles' behavior is the hull function, which is often used to characterize the incommensurate and commensurate phases [92, 180, 188, 218]. The 2D hull function has two spatial dependencies [64]. To verify whether or system is unpinned, we use the approximated method by ref. [153], and take into account only one direction. In this method $g(x)$, the function is calculated for only one single row of particles in $x$ direction. In the state is unpinned, $g(x)$ has continuous form; and when it is pinned, $g(x)$ shows jumps [64]. Our results indicated that the slider is far below Aubry transition, i.e. the state is unpinned.

\section{$2.3 \quad$ Eigenmodes}

Eigenmodes analysis. Eigenmodes analysis gives information about accessible equilibrium modes for the system at hand, presuming that harmonic 
potentials stabilize the system. It has a wide range of usage in studying classical physical behavior such as vibrational spectra at the atomic scale and also transport properties in solid-state physics [219]. To analyze the vibrational excitation in the slider, we calculate the eigenmodes induced in its lattice while moving, which has been used in many other studies [89, 150, 220-223]. For the slider of $i=1, \ldots, N$ particles, number of available eigenmodes are $k=1, \ldots, d N$, and $d=2$ the spatial freedom (dimension), each with its own characteristic frequency $\omega_{k}$. The eigenmodes calculated from the massweighted hessian matrix ${ }^{3}$ and contains all particle-particle potentials:

$$
H_{\mu \nu}=\frac{1}{\sqrt{m_{\mu} m_{\nu}}} \frac{\partial^{2} E}{\partial \mu \partial \nu}
$$

where $E$ is the system's total energy calculated with Eq. 2.1 (sum over all particle-particle interaction), and the derivatives evaluated with the slider's particles are in their intact equilibrium lattice positions (in this calculation we excluded the interaction with the rigid potential sources in the surface for calculating the hessian matrix). The indices $\mu, \nu$ indicate all the Cartesian coordinates for the particles in the slider, and $m_{\mu, \nu}$ are the mass for those particles. With diagonalizing the hessian matrix, a class of eigenvectors $\vec{\xi}_{k}$ is acquired, which describes the direction the ions move, it is known as the polarization vector of normal mode, and each has an characteristic eigenfrequency $\omega_{k}^{2}$. For the $2 \mathrm{D}$ slider's lattice with periodic boundary conditions, we always have two modes zero-frequency which are corresponding to the universal translations in the $x$ and $y$ directions. Besides, when the slider is obc, there is another mode which has zero-frequency corresponding to a universal rotation. In this approach, if the lattices are fully periodic and also defectfree crystalline structures (i.e. having a transnational symmetry), instead of saying a normal mode with wave vector $k$ is excited, we can say there are phonons with wave vector $k$ in the system [224, Chapter 23].

While simulating the sliding, for each particle, we captured $\vec{u}_{i}(t)$ and $\vec{v}_{i}(t)$, which are the displacement of each particles from the initial coordinates in the perfect lattice, and velocity of the particles, all as functions of $t$ the simulation time, respectively. Moreover, for the eigenmode number $k$, we can calculate the kinetic energy:

$$
K_{k}(t)=\frac{1}{2}\left(\sum_{i=1}^{N} \sqrt{m_{i}} \vec{v}_{i}(t) \cdot \vec{\xi}_{k, i}\right)^{2},
$$

\footnotetext{
${ }^{3}$ Hessian is a square matrix in which each elements is the second-order partial derivatives of a scalar function with respect to geometry.
} 
in this relation, the sum is over whole particles, and each particles $i$ has mass $m_{i}$ and $\vec{\xi}_{k, i}$ the vector for particle $i$ of the total eigenvector $\vec{\xi}_{k}$. By this definition, we can conclude:

$$
E_{\mathrm{kin}}=\sum_{i=1}^{N} \frac{m_{i} \vec{v}_{i}^{2}}{2}=\sum_{k=1}^{d N} K_{k}
$$

and it holds completely for both anharmonic and harmonic sliders. For the rest of this chapter, we will use mode instead of eigenmode.

Mode shape. For an oscillating system, when there is high symmetry, a mode shape is a pattern of motion in which all the particles of the system move with the same frequency and fixed phase relation; in other words, "it is a polarization vector field that describes the magnitude and direction of the vibration of atoms in a phonon" [225]. Based on this definition, the free movement of the system described by the modes takes place at fixed frequencies. Each mode is characterized by a mode frequency (or eigenfrequency, $\omega_{k}$ ) and a mode shape $\left(\vec{\xi}_{k}\right)$. A mode shape is a deformation that the component would show when vibrating at the eigenfrequency. The superposition of the modes is the most general motion of the system. In a dynamical system, several modes can be exhibited and often simultaneously [219, 225, 226].

Mode shapes illustrate the relative displacement of two or more particles. These can be very simple when all the particles move in one direction with the same amplitude as longitudinal $(k=0)$ or transversal $(k=1)$ mode [Fig. 2.1], or it could show a highly complex shape for a $2 \mathrm{D}$ system vibrating at high frequencies [Fig. 2.2]. The energy of these modes [Eq. 2.5] could be degenerate, and based on their frequencies, they can absorb energy and get excited.

Resonance. When the frequency of an applied force to an oscillator is equal to or close to its natural frequency, its amplitude will increase. Resonance occurs when the response of an oscillator to being driven at this frequency is an increase in the amplitude. An oscillator is, therefore, said to be resonant at its eigenfrequency [227]. For a one-dimensional chain of coupled particles with mass $m$, sliding over periodic potential (e.g. in the 1D FK model), "washboard frequency" is the frequency "at which the crests of the sinusoidal potential move by". Besides, the discreteness of the chain could lead to several resonances. Resonance for this chain occurs when the washboard frequency is equal to the chain's eigenfrequency of the phonon $[181,228]$. 


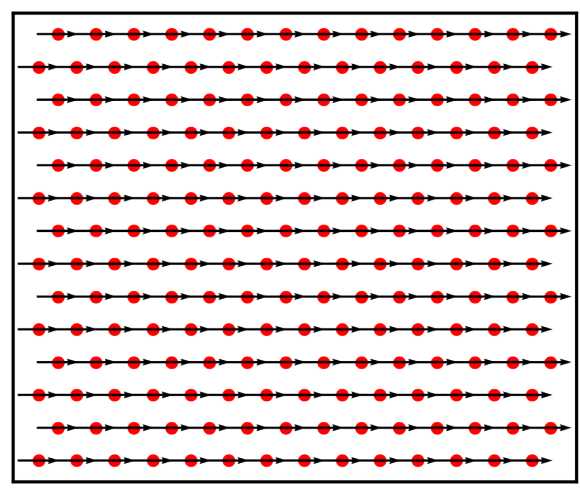

(a) Longitudinal mode: $\vec{\xi}_{0}$

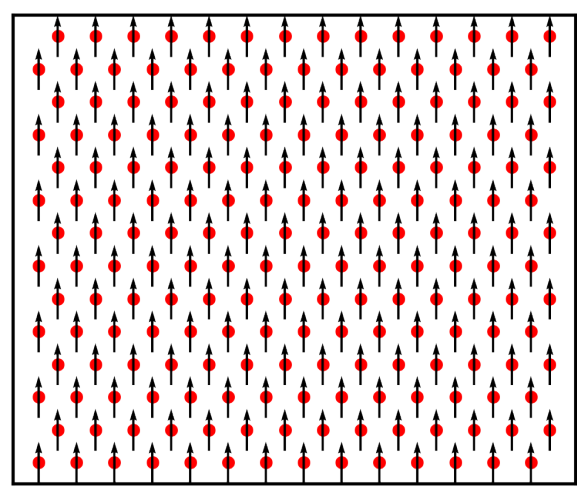

(b) Transversal mode: $\vec{\xi}_{1}$

Figure 2.1: Mode shapes for the pbc. (a) and (b) show two modes of the system with zero-frequency. The solid red circles illustrate the position of the particles in the slider, and the particles' displacements in those modes are shown by the black arrows. The size of the arrow also presents the amplitude of displacement. In (a) and (b), all the particles have the same amplitude, frequency, and move in one direction.

For the harmonic pbc, we presume that the rigid potential sources cause a modulation having characteristic wave vector following $k^{\star}=2 \pi / s$. And $s=a_{b} \sin 60^{\circ}$ is the distance among rows of potential sources at $60^{\circ}$ with respect to $x$-axis [Fig. 2.4]; the associated vibrational frequency can be determined from by finding the dispersion relation:

$$
\boldsymbol{D}(\vec{k})=\frac{2 \alpha_{2} \epsilon}{m} \sum_{i=1}^{3}\left[1-\cos \left(\vec{k} \hat{n}_{i} a_{s}\right)\right] \hat{n}_{i} \otimes \hat{n}_{i},
$$

where $\boldsymbol{D}$ is the dynamical matrix, $\hat{k}$ is a wave vector in the first Brillouin zone, and $\hat{n}_{i}$ are the unit vectors shown in the Fig. 2.4(b); $m$ is mass of the particles and assumed to be the same for all of them. The eigenfrequencies are the square root of the eigenvalues of the dynamical matrix (Eq. 2.7):

$$
\omega_{1,2}(\vec{k})=\frac{1}{\sqrt{2}}\left[D_{11}+D_{22} \pm \sqrt{\left(D_{11}-D_{22}\right)^{2}+4 D_{12}^{2}}\right]^{1 / 2}
$$

where $D_{i j}$ is the $i j$ element of the dynamical matrix for each wave vector $\vec{k}$ [229].

We assume that for the polarization and direction, must dominants modes are longitudinal modes propagating at $\pm 30^{\circ}$ respective to $x$. The corresponding vibrational frequency, $\vec{k}$, in the longitudinal branch will be $\omega_{i}^{\star} \simeq$ 


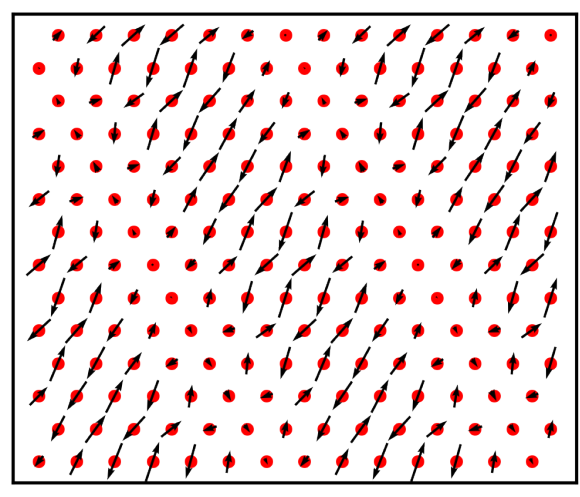

(a) $\vec{\xi}_{150}$

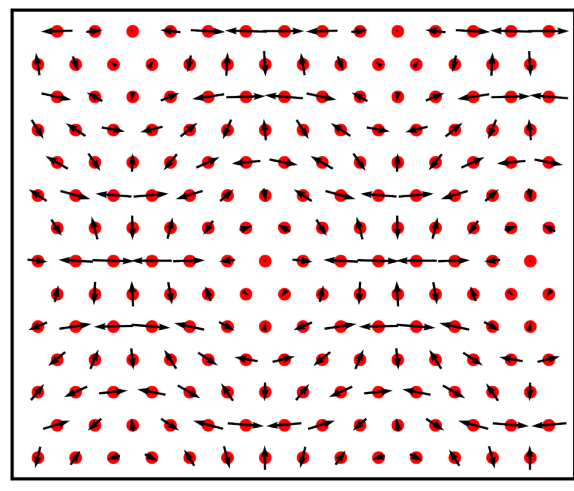

(b) $\vec{\xi}_{382}$

Figure 2.2: Mode shapes for the pbc. (a) and (b) show two modes of the system with high frequencies. same as Fig. 2.1 the circles and arrows display the particles and their relative displacement respectively. In (a) and (b), particles have different directions and amplitudes in the respective mode.

20.263, 9.018, 16.250, 0.0 [Fig. 2.3]. Using these values to calculate the resonances velocities by the washboard relation $\Omega=v_{\text {kick }} / a_{b}$ we have $v^{\star} \simeq$ $2.052,0.913,1.646,0.0$, respectively. Now we will look at the decay of the COM velocity and the energy of the mode during the decay for the three non-zero resonance velocities.

\subsection{Periodic boundary conditions slider}

The pbc system is a fully periodic slider in both $x$ and $y$ directions, with $N=196$. It was set on the top of the surface and orientation is like the one shown in Fig. 2.4. At beginning, when $t=0$, we "kick" the slider in the $x$ direction; for this all the slider's particles are given the velocity $v_{\text {kick }}$ (before assigning the velocity, the whole slider is randomly displaced to sample different initial positions).

The system is completely $2 \mathrm{D}$, meaning that particles in the slider can move and vibrate in the lateral directions. Initial the motion is solely in the direction of kicking, but very soon after, the slider exhibits motion in the $y$ direction as a result of facing the potential sources. 


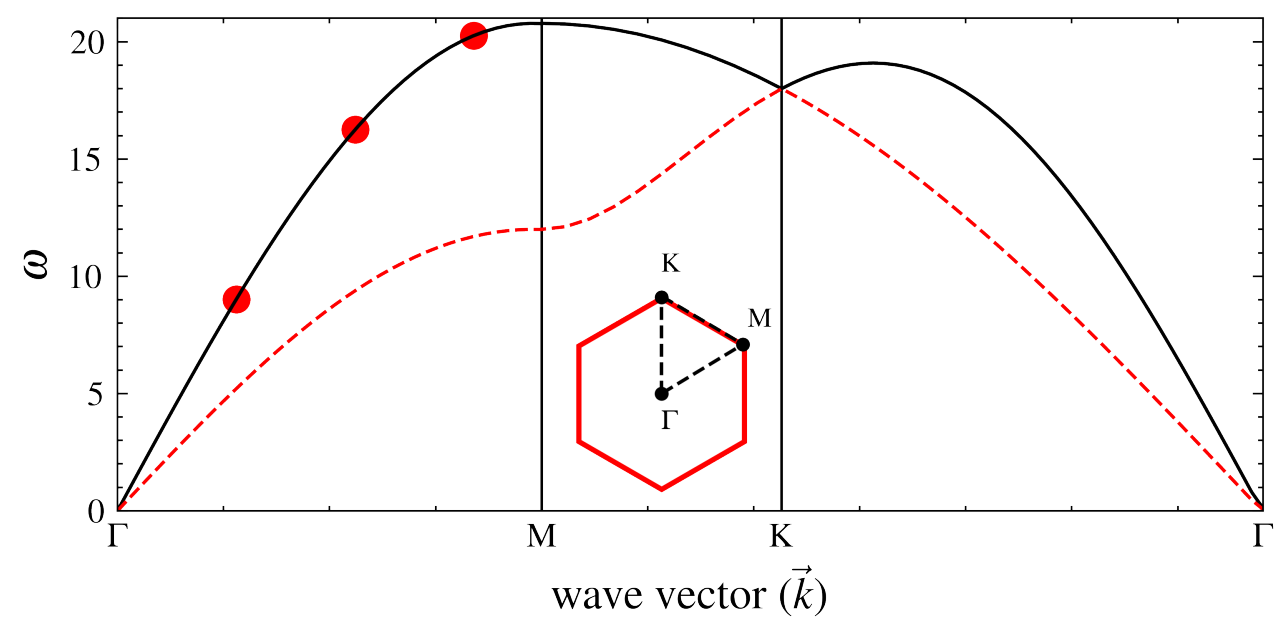

Figure 2.3: Phononic dispersion of triangular lattice with lattice space equal to the unit. The black solid curve shows the longitudinal frequencies, and the red dashed line shows the transverse frequencies. The red hexagonal inset shows the direction in the first Brillouin zone. The solid red circles on the longitudinal frequencies denote the washboard frequencies; from which we calculated the resonance velocities $\left(v^{\star}\right)$.

\subsubsection{Decay behaviors}

Based on the results from previous section, a pbc slider kicked velocity $v_{\text {kick }}^{\star}$ in shared crystalline axis (the $x$-direction), the lattice space of the potential surface $a_{b}=7 a_{b} / 11$ (Fig. 2.4). When the slider is kicked with the $v_{\text {kick }}^{\star}$ the resonance made the $\mathrm{COM}$ velocity decay rapidly, i.e. the friction is high [Fig. 2.5(c)]. Fig. 2.5(c) shows that the $v_{\mathrm{COM}}$ started to decay almost immediately after kicking, decaying very fast. At the beginning of the decay, only several modes with $\omega=20.263\left(=\omega_{1}^{\star}\right)$ are highly active, and then the energy is dissipated among other modes and slider is thermalized. Also, the figure indicated that the activation of few modes is enough to make the $v_{\mathrm{COM}}$ decay. To understand the modes' behavior, we kicked the slider with off-resonance velocities. First, the slider was given a very low onset velocity equal to 0.5 . The result is shown in the figure. This low velocity inserts a very small amount of energy into the slider. This energy is transferred mostly to the most active modes. As we can see here, the most active modes at the beginning are those with $\omega=\omega_{1}^{\star}=20.263$.

For the harmonic slider, at the resonance kick velocity $v_{\text {kick }}^{\star}=2.052$ the decay of $v_{\mathrm{COM}}$ with time is shown in Fig. 2.5(c), and Fig. 2.5(b) indicate that the behavior for $v_{\text {kick }}=0.05$, i.e. much smaller the resonance velocity. At 

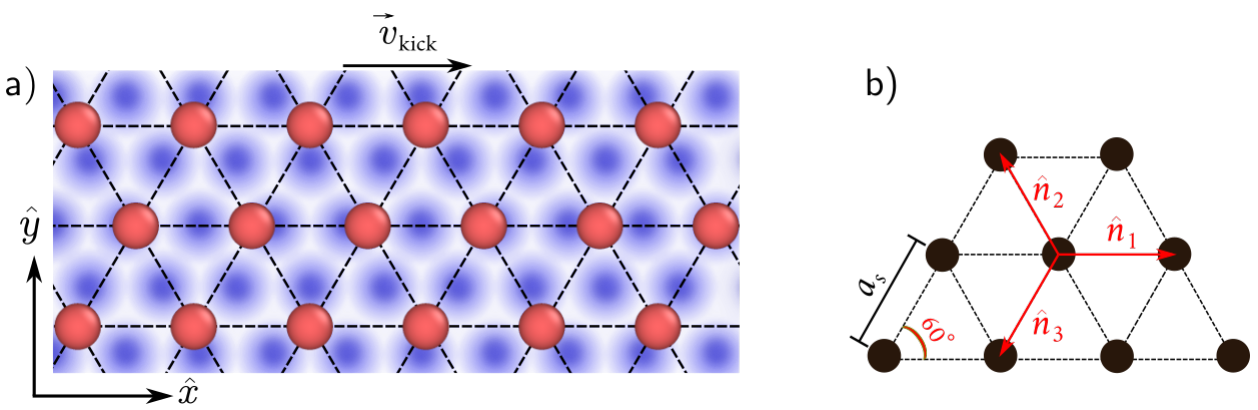

Figure 2.4: a) A schematic view of the system configuration: The red spheres are resembling the particles in the slider, where springs shown by dashed lines connect them to their neighbors. The substrate is denoted by the blur blue circles under the slider. b) It shows the the lattice unit vectors, $n_{i}$, for the slider.

resonance we have a fast decay, meaning that friction is very high. Moreover, initial activity of the modes with $k^{\star}$ are extreme, showing the assumption that the COM motion couple most strongly to the longitudinal modes which propagating at $\pm 30^{\circ}$. There is a state for the slider where $v_{\mathrm{COM}}$ is almost zero, still the modes are not thermalized [Fig. 2.5(b)]. When $v_{\mathrm{COM}} \sim 0$ slider shows no more displacement. Redoing the simulation with $v_{\text {kick }}=0.05$ and using anharmonic interaction in the slider, Fig. 2.5(c), we notice a somewhat faster decay of $v_{\mathrm{COM}}$ then the slider with harmonic interaction kicking with the same $v_{\text {kick }}$ but in anharmonic case, the system completely thermalizes, in other words, all modes show activity. 

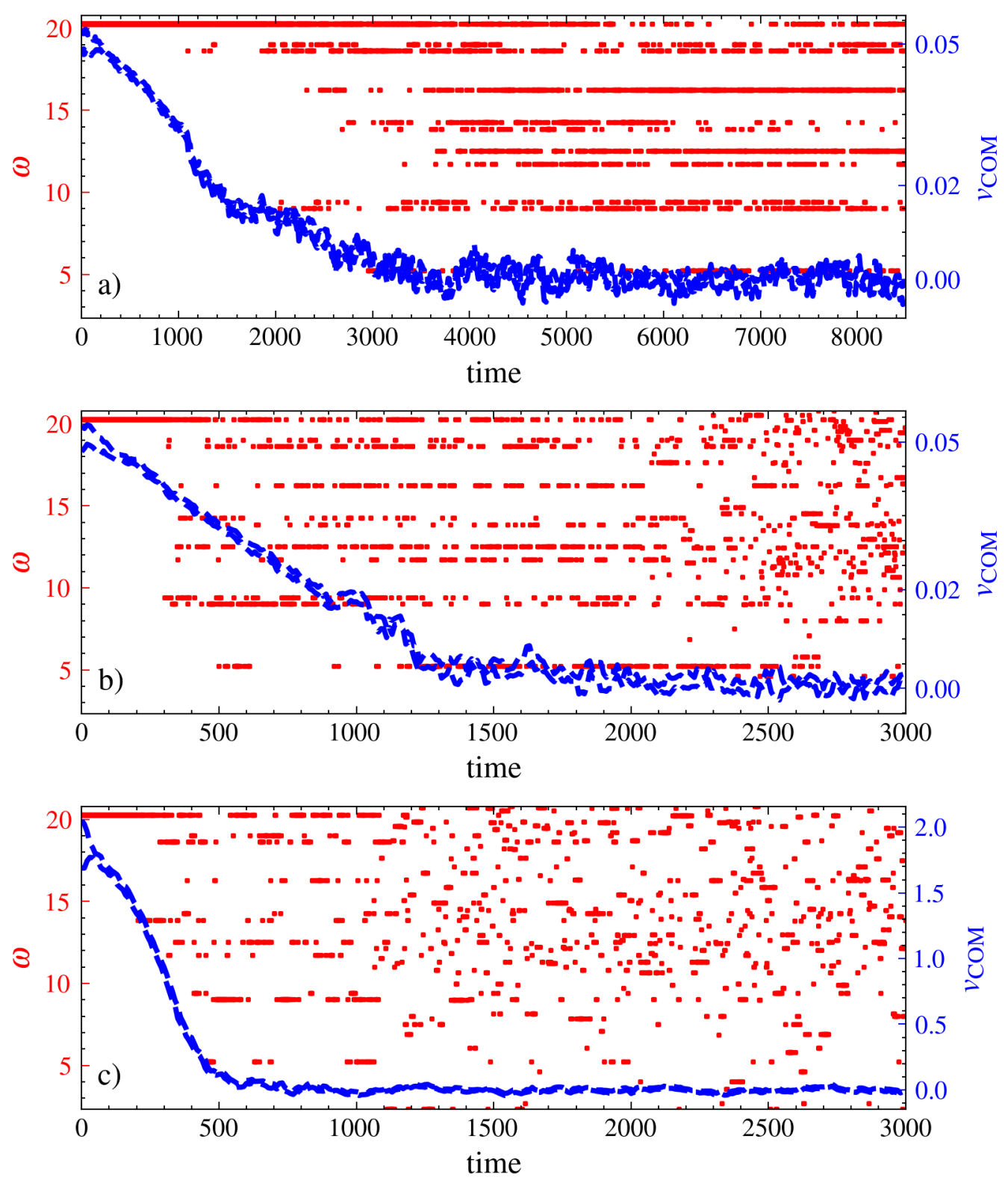

Figure 2.5: Sliding behavior of the pbc, indicating time evolution soon after been kicked with initial velocity $v_{\text {kick }}=0.05$ (over 20 distinct trajectories of the slider were averaged to get these results, and each was initiated with a displaced position). The upper and lower (envelope) of the $v_{\mathrm{COM}}$ of the slider's center of mass is shown by dashed blue curves. The frequency of the most active mode at each time step is indicated by the red dots. (a) showing the results for harmonic pbc with $v_{\text {kick }}^{\star}=2.052$ (b) and (c) for harmonic and anharmonic bonds with $v_{\text {kick }}=0.05$ (See Fig. A.3 for the heatmap version of these results). 


\subsubsection{Coefficient of friction}

In the present work, as a function of time, we observe different paths by which the kicked slider losses its $v_{\mathrm{COM}}$ and stops; for instance, depending on the $v_{\text {kick}}$, these paths have exponential form, linear, or in many cases, have reverse "S" shapes. The rate at which the slider loses its $v_{\mathrm{COM}}$ is referred to as the coefficient of friction (COF). This coefficient is one of the critical parameters when analyzing the tribological characteristics of the tested system [230]. Measuring the COF can give an idea about how fast or slow the decay is; for this, we looked for a mathematical function that can fit all the different curves and in which we can compare the different coefficients of friction.

Sigmoid curve. We fitted $v_{\mathrm{COM}}$ to a mathematical function to quantify its relation with $v_{\text {kick }}$ and its decay overtime to find the corresponding COF. Preferably, this function's fitted curve would be the actual curve calculated from simulation, which expresses the $v_{\mathrm{COM}}$ as a function of $v_{\text {kick }}$ and time. Even though the decay of $v_{\mathrm{COM}}$ has an exponential form, we need a function that could describe the curve better because of the plateaus at the beginning of sliding in many cases. The calculation of approximating this function is known as a curve model. This model, in most cases, gives details about a class of curves through parameters. Then the function fitted to the data by modifying those parameters in order to achieve the closest curve out of the class, which could represent the data better. The usual "S" shaped curves featured two plateaus and a transition region [Fig. 2.6]. A "sigmoid" function describes this class of curves well; it is a mathematical function with a characteristic "S"-shaped curve [App. A.2]. The sigmoid function ideally originates at $(0,0)$, has a point of turning at the early growing state, and is either reaching the highest value, an asymptotic or rising and dropping in the later state [231]. Nevertheless, the simple sigmoid function can not describe all the curves we have observed. Hence, we used a modified sigmoid function known as the five-parameters logistic function with double slopes (5PL2S) [232-235].

Logistic function. A well-known modification of the sigmoid function is the logistic function. The generalized form of the logistic function is mainly developed in growth studies: a branch of science dealing with complex nonlinear growth [231]. The basic form of the logistic function is known as four parameters logistic (4PL) function [236]; and has the following nonlinear form:

$$
Y=f(X ; \mathbf{p})=f(X ; a, b, c, d)=d+\frac{a-d}{1+\left(\frac{X}{c}\right)^{b}},
$$


where parameters vector $\mathbf{p}$ of the $4 \mathrm{PL}([a, b, c, d])$. $Y$ is the dependent variable, and $X$ is the independent one (for our data $Y$ is $v_{\mathrm{COM}}$ and $X$ is time). In this function, $a$ is the highest value, $d$ is the minimum, $c$ is the halfway between the upper and lower plateaus (i.e. the curve's inflection point), and $b$ is the maximum slope of the decay [231, 237] which should be estimated [Fig. 2.6].

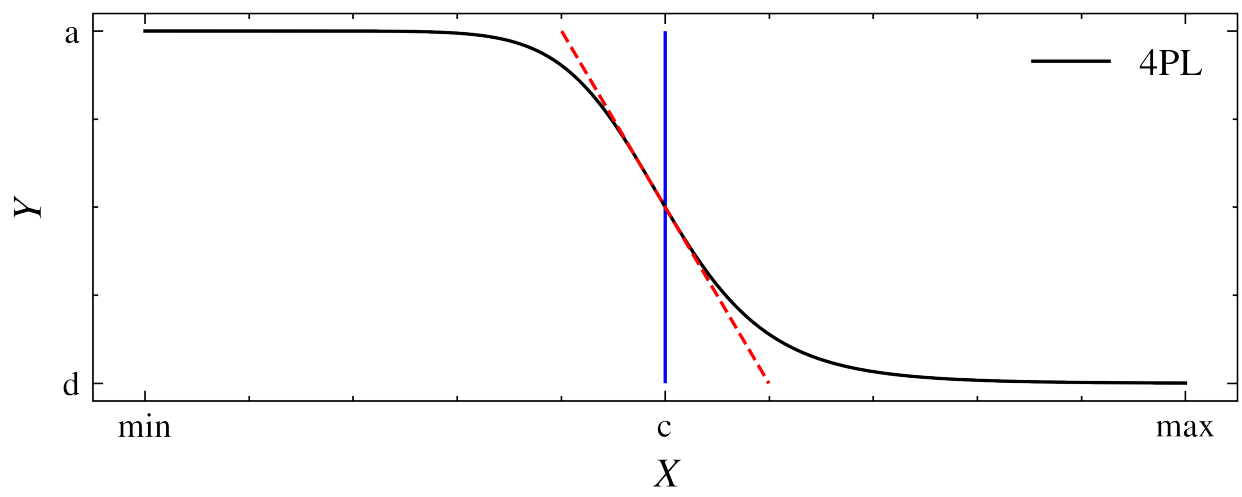

Figure 2.6: The curve with typical sigmoidal behavior; here the curve is a four parameters logistic function (4PL) [Eq. 2.9]. The blue vertical line shows the halfway of decaying and the red dashed line indicates the maximum slope of the decaying ( $b$ in Eq. 2.9).

The $4 \mathrm{PL}$ is symmetric about $(X=) c$. The symmetric $4 \mathrm{PL}$ function does not generally describe the discovered behavior in the simulated system; thus, an asymmetric modification of the 4PL is necessary to have a better approach. Using the log of 4PL allows us to deal with "asymmetry" behavior in curves. Logarithm have a better description of the asymmetrical behavior, and can enhance the fit at the shorter "tail" of a sigmoidal curve with but not at the upper side of the curve. Still, we can improve the 4PL by using the fifth parameter that take care of the asymmetry of the curves, which is referred to as the five-parameters logistic function (5PL):

$$
Y=f(X ; \mathbf{p})=f(X ; a, b, c, d, g)=d+\frac{a-d}{\left(1+\left(\frac{X}{c}\right)^{b}\right)^{g}}
$$

here $\mathbf{p} \equiv[a, b, c, d, g]$ being the vector for the parameters of the 5PL function. The only restriction on the parameters is that $c$ and $g$ must be bigger than zero. By setting $g=1$, the $4 \mathrm{PL}$ is obtained [Fig. 2.7] [233]. The parameter $b$ controls the curves when it approaches the $a$ plateau, and along with $g$, their product $b g$, adjust the rate of approaching the $d$ plateau. 
In $5 \mathrm{PL}$ form, the halfway through the decay is $X_{\text {halfway }}=c\left(2^{1 / g}-1\right)^{1 / b}$. One downside of fitting with 5PL is that it is often possible to widely reached to the 5PL parameters so that the curve hardly changes, but the calculated fitting parameters differ [233].

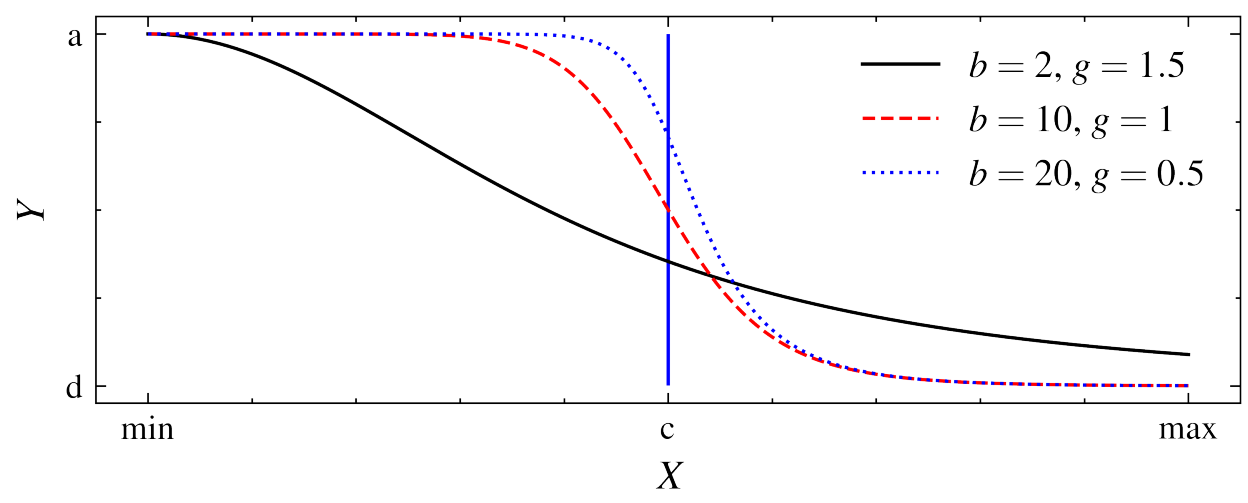

Figure 2.7: The figure shows three curves plotted with 5PL with different sets of parameters in the Eq. 2.10. For this curves $a=0$ and $d=20$. The blue-solid vertical line shows the mid point of the data. This figure also shows that $c$ is not the halfway of the decay for the black-solid and the blue-dotted curves. Also, $b$ is the maximum slope of the transmission from first plateau to the second one, and $b g$ is the slope of transition part to the second plateau.

Eq. 2.10 can be rewritten by assigning two parameters to give a better description of the curvatures. This modification was first used by F. J. Richard [238] is known as the "five-parameters logistic function with double slopes" (5PL2S). By introducing the parameters $\eta, \mu, \mu_{2}$, the 5PL can be rewritten in the following form $[232,239]$ :

$$
Y=f(X ; a, b, c, \mu, \eta)=a+\frac{a-d}{1+f_{X}\left(\frac{X}{c}\right)^{-\mu}+\left(1-f_{X}\right)\left(\frac{X}{c}\right)^{-\mu \eta}},
$$

here $\eta=\left|\mu_{2} / \mu\right|$ is the ratio of the slopes; $\mu$ is the slope of the transition part (from the first plateau to the second plateau) at leaving the first plateau, and $\mu_{2}$ is the slope reaching the second plateau. With this modification $c$ shows the halfway between the upper and lower plateaus. The function $f_{X}$ is a logistic weighting function which is varying smoothly:

$$
f_{X}=\left(1+\left(\frac{X}{c}\right)^{\bar{\mu}}\right)^{-1}
$$

where $\bar{\mu}=2|\mu| \eta /(1+\eta)$ is the average slope of decay (i.e. COF). The main idea behind this modification is to control the function responses while $\mathrm{X}$ passes halfway toward one of the two ends of the data [232]. 
Fitting $v_{\mathrm{COM}}$. When fitting the decay of $v_{\mathrm{COM}}$ with the Eq. 2.11 the maximum of the graph, $a$ corresponds to the initial velocity $v_{\text {kick }}$, and $d=0$, so we can rewrite the equation by subsituing $X$ with time $(t)$ and $Y$ with $v_{\mathrm{COM}}$, as:

$$
v_{\mathrm{COM}}=\frac{v_{\mathrm{kick}}}{1+f_{t}\left(\frac{t}{c}\right)^{-\mu}+\left(1-f_{t}\right)\left(\frac{t}{c}\right)^{-\mu \eta}},
$$

where $f_{t}=\left(1+(t / c)^{\bar{\mu}}\right)^{-1}$. The derivative of the actual $v_{\mathrm{COM}}$ curve shows the slope of the curve at each time. The minimum derivative ${ }^{4}$ of the decay curve is a reasonable estimation of the average slope of decay. We used the time of this minimum as the initial guess for $c$. For $\eta$, we calculate the curves' curvatures, $\kappa=v_{\mathrm{COM}}^{\prime \prime} /\left(1+v_{\mathrm{COM}}^{\prime 2}\right)^{3 / 2}$, which is a ratio between the curve's derivative and its second derivative. The curvature has a minimum in the first end of the first plateau and a maximum at the beginning of second plateau [Fig. 2.8]. The absolute ratio of these two values is a reasonable estimation for the $\eta$ to initiate the fitting procedure. In Fig. 2.8, the decay of $v_{\mathrm{COM}}$ for the harmonic pbc (the slider we studied in the previous section) with $v_{\text {kick }}=1.168$ is plotted, which illustrates a nearly symmetrical trend in approaching its plateaus.

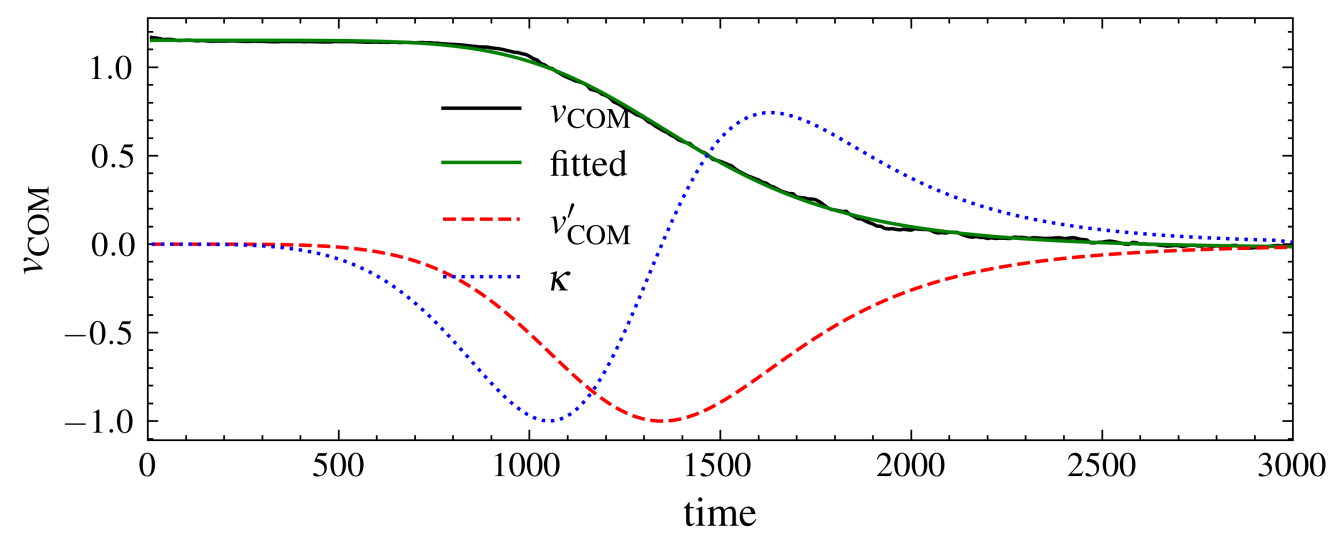

Figure 2.8: The decay of $v_{\mathrm{COM}}$ with its derivatives for a harmonic pbc kicked with $v_{\text {kick }}=1.168$. The solid black line shows the decay of the $v_{\mathrm{COM}}$, the solid green line shows the fitted curve with Eq. 2.11, the blue dashed curve illustrates the derivative of the decay, and the dotted red curve shows the curvature, $\kappa$, of the decay. The values of the derivative and curvature were normalized to one in order to see their extrema.

\footnotetext{
${ }^{4}$ It has been proven that a better way to estimate initial parameters is using derivatives which shows more accurate parameter estimation than computational approaches. [e.g. see Ref. [231, 240]].
} 
The derivative of the decay, $v_{\mathrm{COM}}^{\prime}$, alongside its curvature's curve, $\kappa$, are shown in Fig. 2.8. The curve of the $\kappa$ exhibits two extrema while the $v_{\mathrm{COM}}$ is approaching the plateaus: a minimum while $v_{x}$ left the first plateau and a maximum when it reached the second plateau. By using this information, the $v_{\mathrm{COM}}$ was fitted to Eq. 2.13, and the result is plotted with a solid green curve in Fig. 2.8.

By looking at the time-evolution of the $v_{\mathrm{COM}}\left(=\sqrt{v_{x}^{2}+v_{y}^{2}}\right)$ the slider [Fig. 2.9] and its component, it is clear that the first extremum of the $\kappa$ is when the $v_{x}$ of slider begin to decrease. Then, with the beginning of the decay, the $y$ component of velocity increases, and the slider takes up movement in the other direction; this can be seen from the rise in $\sqrt{\operatorname{msd}_{y}}$ (msd is mean squared displacement). The second extremum happens when the slider stops from further coherent motion in any direction. The fitting parameters for this velocity are shown in Tab. 2.1. The high value for $|\mu|$ means that the transition from the first plateau to decaying region is sharp. Also $\eta$ is comparably high, indicating that the transition to the second plateau is also quick. The minus sign represents the decrease in velocity.

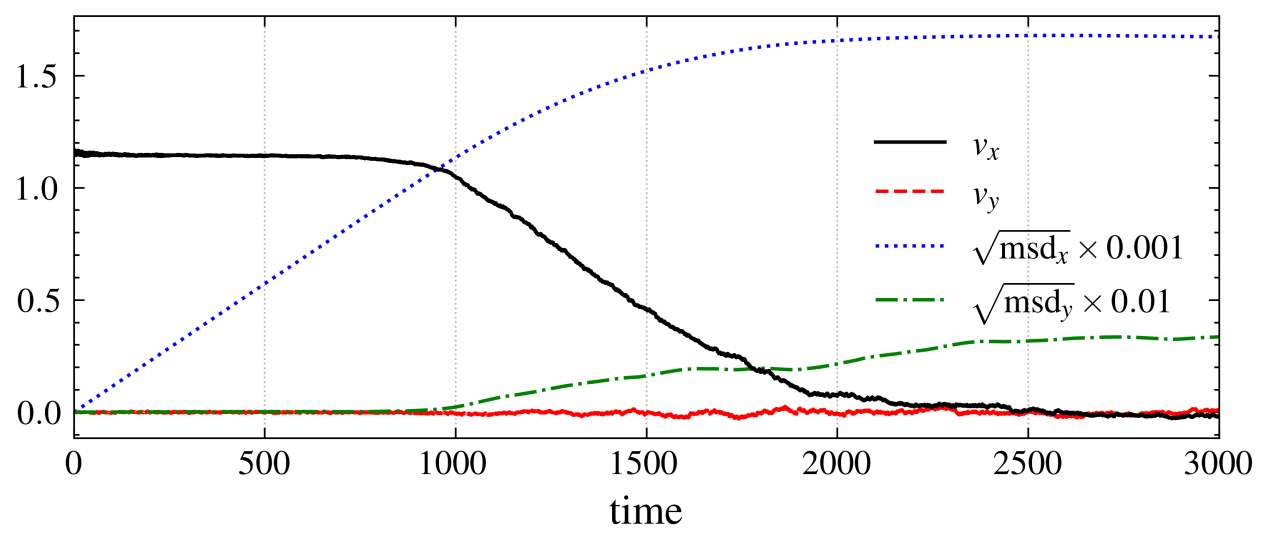

Figure 2.9: Time evolution of the harmonic pbc with time initiated with $v_{\text {kick }}=1.168$. The $y$ axis of the figure shows the scale values based on each curve as a function of time (all the data are in dimensionless reduced units). The solid black curve shows the decay of the $x$ component of $v_{\mathrm{COM}}$; also, the $y$ component of $v_{\mathrm{COM}}$ is plotted as red dashed curve. The blue dotted curve and the green dash dotted curve illustrate the square root of displacement in the $\mathrm{x}$-direction $\left(\sqrt{\mathrm{msd}}_{x}\right)$ and $\mathrm{y}$-direction $\left(\sqrt{\mathrm{msd}_{y}}\right)$, respectively; these two values are multiplied by factors so it can be seen in the figure. All the results here are also an averaged value of 20 trajectories of the slider, with distinct initial positions. 
By Fitting the decay of three resonance velocities we found in the previous section $\left(v_{\text {kick }}^{\star, i}=2.052,0.913,1.646\right)$ using the Eq. 2.13, we can quantify the trends of their $v_{\mathrm{COM}}$. The results are shown in Fig. 2.10. The fitted curves are exhibited with solid green curves in the figure. Also, the parameters of the fitting are listed in Tab. 2.1. If kicked with one of these velocities, the harmonic pbc sliding distance is very short compared to the other initial velocities (shown in the table). Although the total sliding distance for each of these kicks is close to each other, still, for each kick, the slider is going through a different decaying path.

Comparing the fitted parameters reveals the differences and similarities of the curves in the Figs. 2.8 and 2.10. When the slider is kicked with $v_{\text {kick}}^{\star, 1}$, the initial plateau is very short, and decay starts at a high rate. Comparing its $\mu$ value with different velocities listed in the table shows that the decay is more than six times faster than the second-highest velocity in the table $\left(v_{\text {kick }}^{\star, 2}\right)$. Also, the absolute value of the average slope of decay, $|\bar{\mu}|$, for $v_{\text {kick }}^{\star, 1}$ is much higher than the one of the other velocities. The low ratio of the slopes also indicates that the rate of the second turning point, when the slider is going to stop, is close to the first turning point. For $v_{\text {kick }}^{\star 2}$, the $\mu$ and $\mu_{2}$ are smaller than the other resonance velocities, which can explain why its $\sqrt{\mathrm{msd}}$ is in the range of the other $v_{\text {kick }}^{\star i}$, even though its initial velocity is low. The high ratio between $\mu$ also indicates that the slider approaches the second plateau faster than it left the first plateau (also for the $v_{\text {kick }}^{\star, 3}$ ). However, for the off-resonance velocity, $v_{\text {kick }}=1.168, \mu$, and $\bar{\mu}$ are the lowest, and $c$ is large, which corresponds to this far sliding of the pbc slider.

Table 2.1: The parameters calculated from fitting the curves in the Figs. 2.8 and 2.10 with Eq. 2.13. In which, $\mu$ is the slope as the data approaches the first plateau. $\eta$ shows the ratio between the slopes, $\bar{\mu}$ shows the average slope of decaying (COF), $c$ is the time of halfway between the two plateaus, and $\sqrt{\mathrm{msd}}$ is the square root of mean squared of the total displacement.

\begin{tabular}{lccccc}
\hline & $\mu$ & $\eta$ & $\bar{\mu}$ & $c[$ time $]$ & $\sqrt{\mathrm{msd}}$ \\
\hline$v_{\text {kick }}^{\star 1}=2.052$ & -1.61 & 2.89 & -2.38 & 292 & 9.24 \\
$v_{\text {kick }}^{\star 2}=0.913$ & -0.13 & 20.53 & -0.26 & 301 & 9.28 \\
$v_{\text {kick }}^{\star, 3}=1.646$ & -0.24 & 22.57 & -0.46 & 427 & 9.28 \\
$v_{\text {kick }}=1.168$ & -0.05 & 125.00 & -0.10 & 1190 & 1855 \\
\hline
\end{tabular}




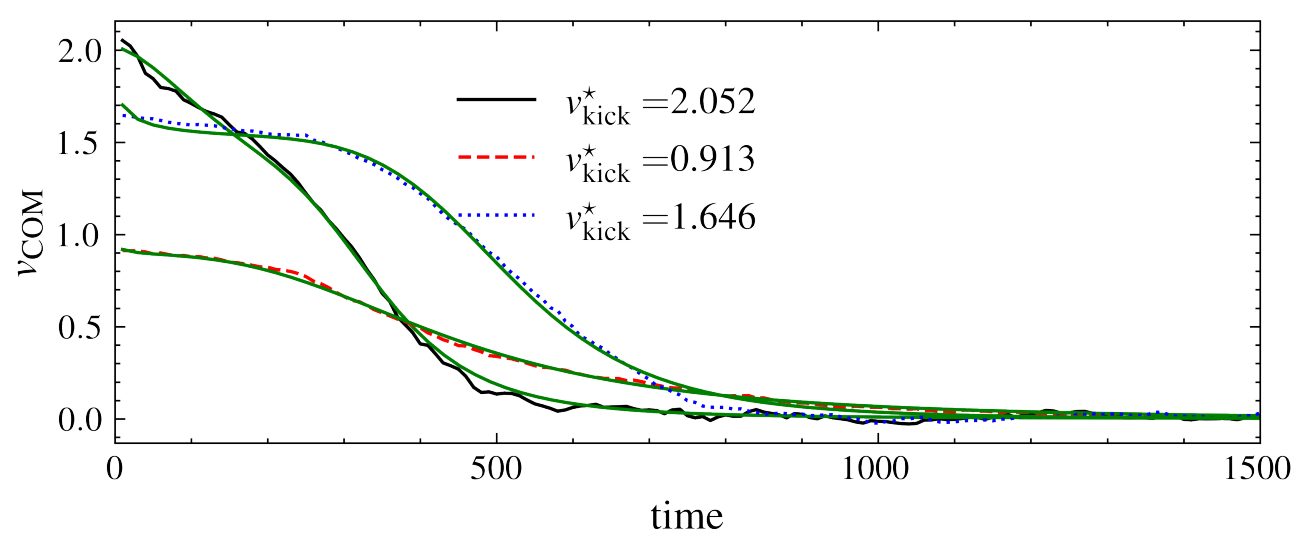

Figure 2.10: The figure shows the decay of the $v_{\mathrm{COM}}$ for three different velocities as labeled in the graph (results here are also an averaged value of 20 trajectories of the slider, with distinct initial positions). The curves fitted with Eq. 2.13 are plotted with the solid-green curve for each $v_{\mathrm{COM}}$ decay. The curves appear to be fitted correctly.

For the anharmonic slider, the initial plateau is relatively shorter than the harmonic slider, indicating that the bonds' anharmonicity can suppress this monotonic behavior at the beginning. Still, the equation we adopted [Eq. 2.13] for the harmonic slider can also fit the $v_{\mathrm{COM}}$ for anharmonic curves. The faster decay of the anharmonic pbc can be seen from their calculated $|\bar{\mu}|$. In general, their $|\bar{\mu}|$ is bigger than the harmonic slider.

\subsection{Open boundary conditions slider}

For the obc system, we consider a circular island cut out of a perfect triangular lattice as the slider which contains $N=199$ particles; which is close to the slider with periodic conditions conditions; particles mass are unit $m=1$. The particles on edge which have less restrictions acts as an extra source of scattering, which dramatically increase friction and reduces the sliding distance. When the initial velocity is small, $v_{\text {kick }}=0.05$, the slider hardly displaced, mostly a vibrating motion of the COM, harmonic or anharmonic interaction does not change this behavior. The damping is strong enough, and thermalization is fast [Fig. 2.12]. In order to see any COM displacements, initial velocity most be much higher then $v_{\text {kick }}=0.05$.

The overall motion of the slider is a superposition of its modes. These modes are the internal degree of freedom (DOF) of the slider, and its motion is highly affected by the dynamics of its DOF. This DOF of the slider can 


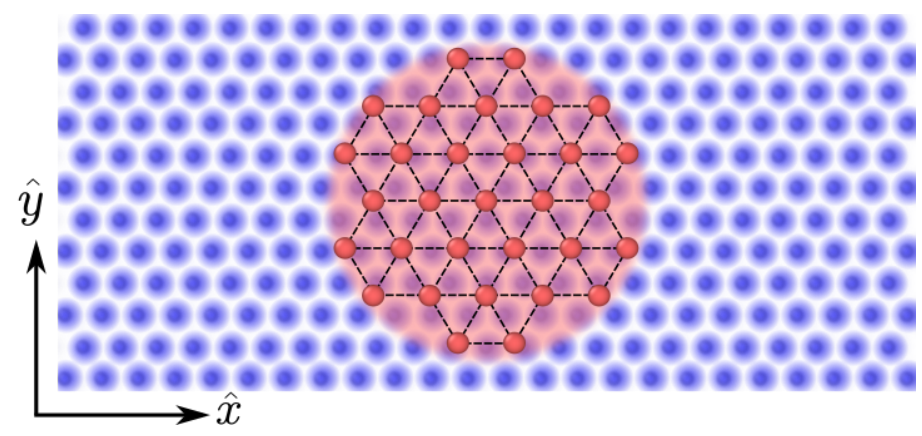

Figure 2.11: A schematic view of the obc slider. The particles in the slider are shown with red circles, and the red shadow area resembles the disk shape of the slider. The dashed lines are showing the bonds between them. The blurred blue circles are showing the potential sources in the substrate.

form an energy reservoir for absorbing and releasing kinetic energy [241243]. Before looking at the decay of the slider started at high velocity, we look at the mode energy spectrum of the harmonic slider with $v_{\text {kick }}=0.05$. Besides the edge of the slider, in this system, the slider and potential surface are free from defects and structural irregularities such as dislocations. In such a system, friction occurs due to structural arrangement and dynamics, referred to as intrinsic friction [243-246].

Standard score. The energy spectra we are studying are made of each mode's energy at that simulation time, from which the average mode energy is subtracted and then divided by the standard deviation. This estimation is known as the standard score or Z-score. This score gives an impression of the distance of a point from the data set's mean.

Not all the modes get the same energy or have the same lifetime [Fig. 2.12]. Some modes dominant the behavior of the slider; for the free slider, modes with rotation, torsion, and breath-like activity have significant effects on the friction of the slider [222, 223].

Breathing, twisting-and-torsion modes. Fig. 2.12 shows the mode activity (as a heatmap) of the slider kicking with $v_{\text {kick }}=0.05$ at each time based on their frequencies. Since the kicking was on the $x$ direction, the first transnational mode with $k=0$ absorbed the most energy at the beginning of the kicking; the energy of this mode and other two modes $(k=1,2)$ were set to zero in Fig. 2.12 to see other modes' activity more clearly ${ }^{5}$. The vibrational patterns of the six modes (basically, the eigenvectors of the slider) are shown

\footnotetext{
${ }^{5}$ This manipulation was kept for all heatmaps in this thesis.
} 


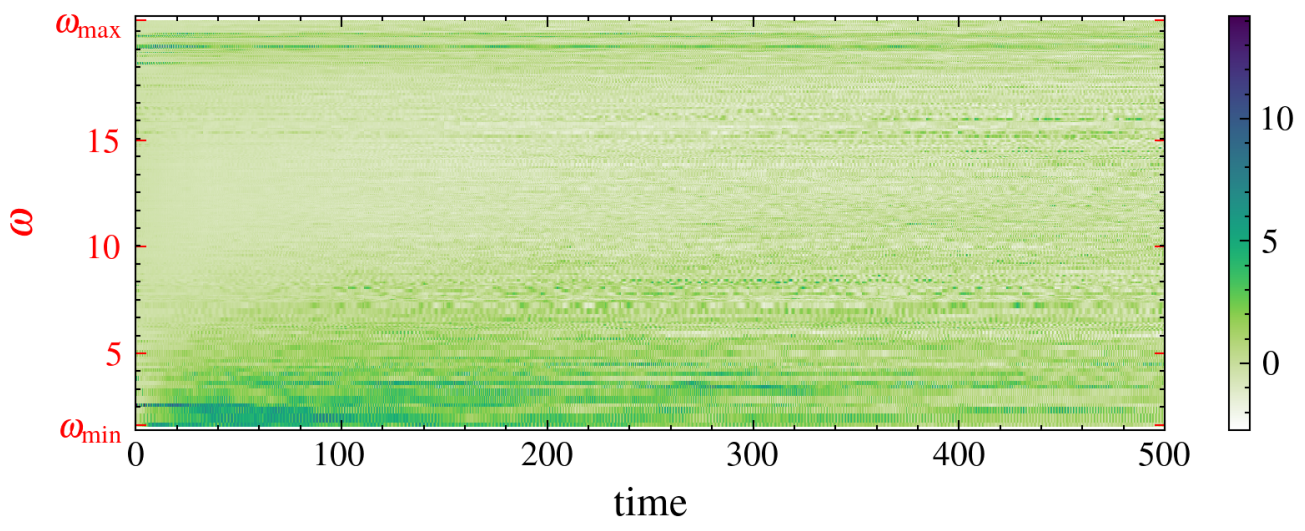

Figure 2.12: Heatmap of harmonic obc with $v_{\text {kick }}=0.05$. The color-coding indicates the mode activity, the standard score of modes at each time step. Most actives modes are those with low frequencies. Before total thermalization (time $\sim 400$ ), only a few modes with higher frequency are very active $(\omega \sim 20)$. The shapes of some of the most active modes are shown in Fig. 2.13.

in Fig. 2.13, distinguished with index numbers. The index numbers are arbitrary; we ordered them based on their eigenfrequencies, $i=0,1, \ldots, 2 N-1$, and $N$ is the number of particles in the slider; a smaller number means a lower frequency. The motion for these modes is indicated with respect to the center of the slider with arrows which are the modes polarization vectors. The dominant behaviors of those modes are breathing-like, torsion, and rotation motions. Although these modes show complex motions, the superposition of highly chaotic behaviors of these modes leads to an overall normal sliding. Fig. 2.13a shows the mode with solely rotation vibration $(k=2)$. Fig. $2.13 \mathrm{~b}$ is one of two eignemodes with the same frequency, $\omega_{3,4} \simeq 1.63$. These two modes have a breathing-like motion with torsion. The mode in Fig. 2.13c is one of few nondegenerate modes with twisting-and-torsion behavior. The direction of arrows on the edge is in counter with the direction of inner particles $\left(\omega_{15} \simeq 3.56\right)$. Fig. $2.13 \mathrm{~d}$ is showing a mode that is mostly active on edge with $\omega_{22} \simeq 4.21$, which is a unique eigenfrequency. The particles on and close to the edge are active; conversely, the inner particle does not show any vibration in this mode. Fig. 2.13e and Fig. 2.13f showing vibrations of two most active modes with high-frequency vibrations; The modes of (e) with $\omega_{353} \simeq 19.3$ has breathing motion in the center of the slider; and (f) with $\omega_{356} \simeq 19.4$ shows very complex vibration including rotation, torsion, and breathing-like motions. Moreover, for modes $k=353,356$, the particles on the edge of the slider have minimal vibration amplitudes. 


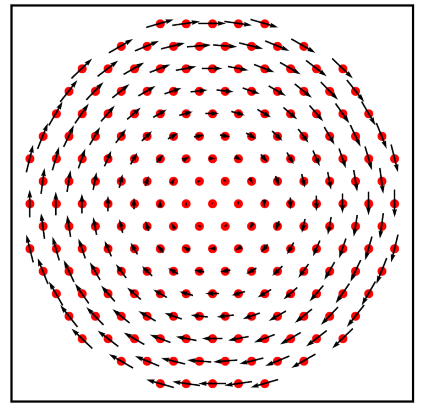

(a) $\vec{\xi}_{2}$

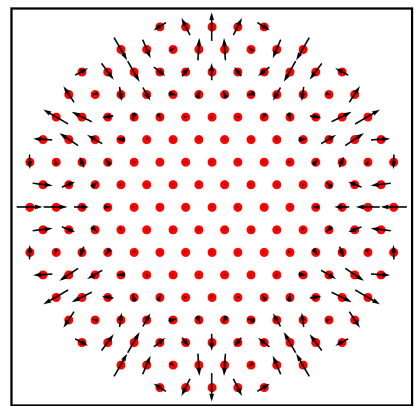

(d) $\vec{\xi}_{22}$

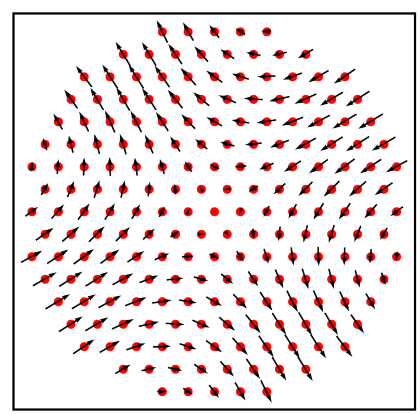

(b) $\vec{\xi}_{4}$

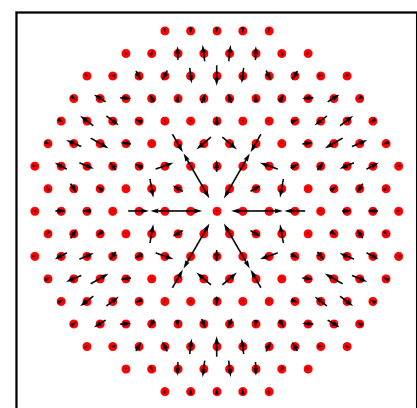

(e) $\vec{\xi}_{353}$

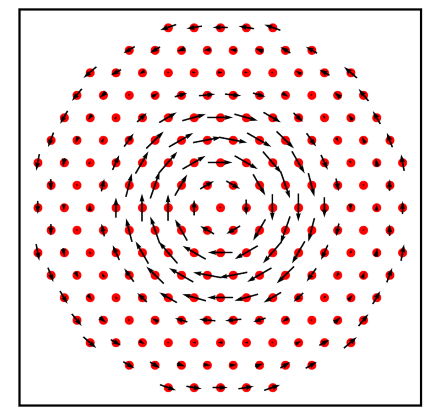

(c) $\vec{\xi}_{15}$

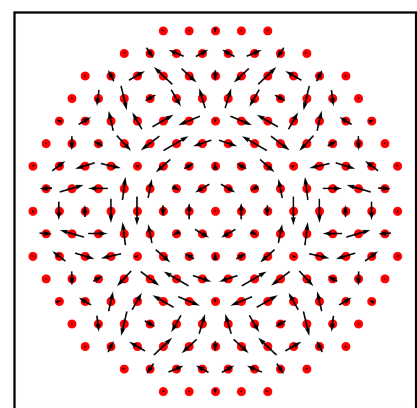

(f) $\vec{\xi}_{356}$

Figure 2.13: The figure shows six of the most active vibrational modes of the harmonic obc slider. The solid red circles show the particles in the obc slider, and the arrows indicate the amplitude and direction of the vibrations.

Immediately after kicking, the first mode $(k=0)$ rapidly lost its energy due to the interaction with the potential surface and spread among other modes. These energies did not spread between all the modes equally. The pure rotation mode [Fig. 2.13a], and the modes with breathing-like behavior [e.g. Fig. 2.13e] absorbed most of the energy at the time. In addition, the modes with low frequencies (generally $\omega \lesssim 5$ ) are mostly are getting active until thermalization. The total energy of the modes is the sum of their kinetic and potential energies. The total energies for six of the most active modes are plotted in Fig. 2.14. The figure shows that the mode with $\omega_{353}=19.3$ [Fig. 2.13e] adsorbed most of the energy immediately after kicking, and while this mode loses its energy, the other modes, especially twisting-and-torsion [219] and breathing-like modes, modes shown in Fig. 2.13a and Fig. 2.13b absorb most of it. The thermalization started when the energy dissipated among other modes. Even though there is no more a dominant mode at thermalization, the energy exchange between modes continues. 


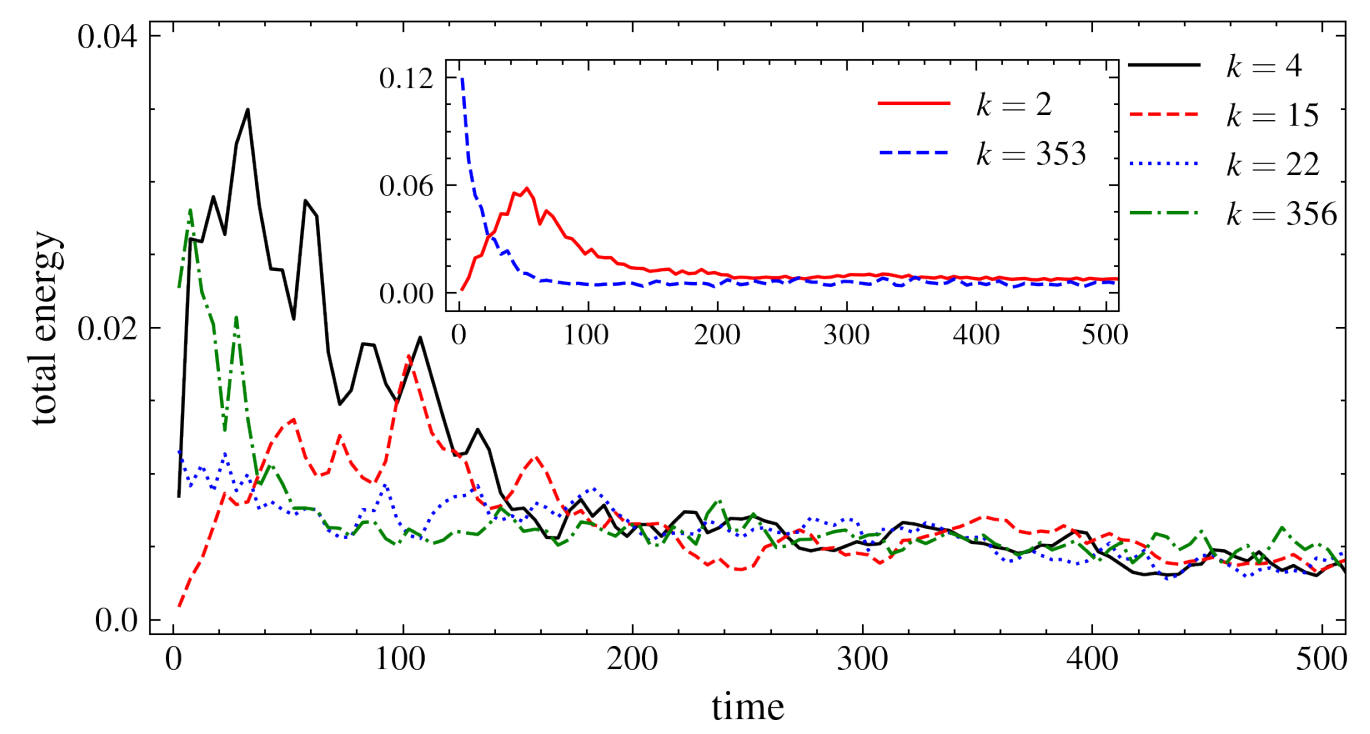

Figure 2.14: Energy of six active modes during sliding for the obc slider with $v_{\text {kick }}=0.05$. The total energy for each mode is calculated at every time step. The curves are labeled with $k$ of the modes. The inset figure is showing the same data for modes $k=2$ and $k=353$.

The time sequence of activation of different modes illustrated that the main contributions to this system's friction are one high-frequency mode $(k=$ $353)$ and a few low-frequency modes. The mode $k=15$ started activation and adsorbing energy later than the other mode. In contrast to the other modes in which their energy dissipate, this mode activation increases, indicating that this torsion mode vibration is highly involved in the friction and initiation of the thermalization.

High initial velocity. In Fig. 2.15, we present results when $v_{\text {kick }}=1$, using harmonic springs. We notice that the thermalization of the system is extremely fast, much faster compare to pbc slider when it was kicked with one of the $v_{\text {kick }}^{\star}$ [cf. Fig. 2.5c]. Also, at the initial mode activity is differ and it is not related to $\omega^{\star} \approx 20.26$ induced by the periodicity of the potential surface, but much lower frequencies are achieve. Since the system thermalized fast, we focus on the first 100 time steps right before total thermalization. From Fig. 2.15, we can see that the modes with moderate frequencies got activated at first. Afterward, their energy dissipated into ones with low frequencies and then dissipated between all the modes. Another point is that the modes with high frequencies which got activated with the lower initial velocity, with higher velocity, losing their energy very fast. 


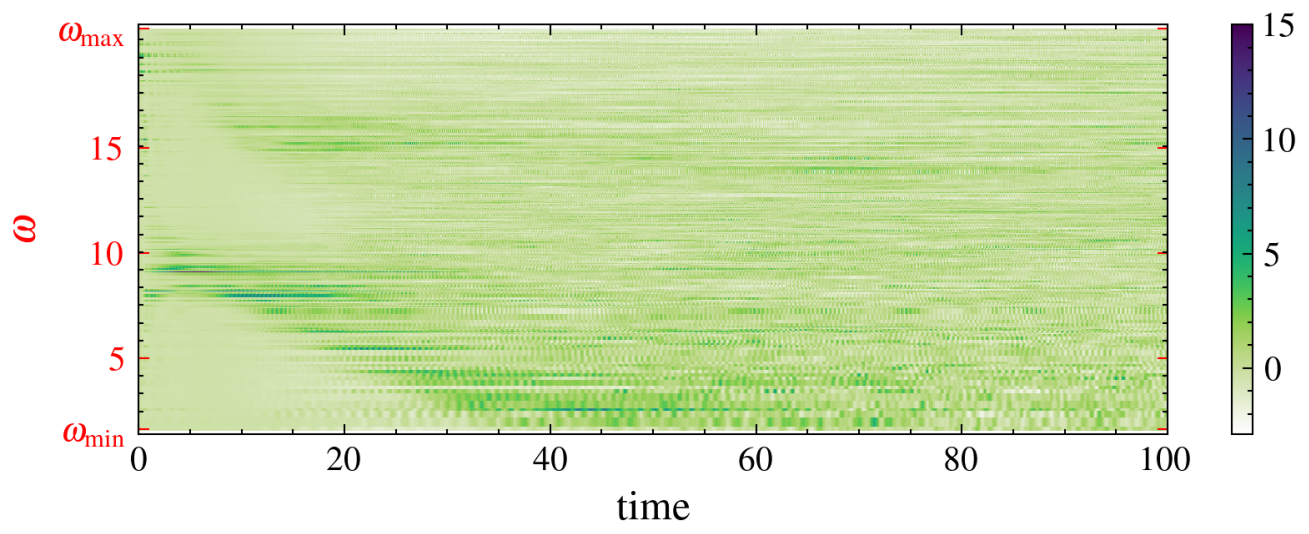

Figure 2.15: Heatmap of harmonic obc with $v_{\text {kick }}=1.0$. The mode activity are shown with color-coding, and the illustration of the data is the same as in Fig. 2.12

Rotational kicking. The mode spectra for direct kicking showed that the pure rotation mode absorbed most energy from those two modes [see also Fig. 2.14]. As a result, the slider started to rotate. To see whether pure initial rotation would lead to any transnational displacement of the system, we can give particles an angular velocity instead of giving the system initial kick in the $x$-direction. For this, each particle $i$ received an angular velocity $\vec{v}_{\text {kick }}^{i}=\vec{r}^{i} v_{\text {kick }}^{\text {rot }}$. Where $\vec{r}^{i}$ is the vector originated from the central particle of the slider toward particle $i$. The slider rotated counterclockwise, even though the direction of rotation does not affect the results. When the slider was kicked in the $x$-direction, the total kinetic energy inserted in the system was $E K=\sum_{i=1}^{199} \frac{1}{2} m_{i} v_{i}^{2}=99.5$ (in which, $m_{i}=1$ and $v_{i}=1$ ). To compare the result from rotational kicking with the direct kicking we set initial velocity equal to $v_{\text {kick }}^{\text {rot }} \simeq 1.34$. With this, the total energy we inserted in the system was 99.5. Besides these changes, the rest of the simulation protocols were the same as before. The result is shown in Fig. 2.16.

Like the direct kicking, here again, mostly the modes with low frequencies are active. Still, in contrast to the direct kicking, there are no dominant modes in the slider in rotation, besides a mode with $\omega_{41}=6.09$ which is very active for a short time [cf. Fig. A.5a]. The initial energy initially is in the rotation mode. This energy immediately transforms into a few more modes (including transnational modes) [cf. Fig. A.4]. This energy transformation between these modes is comparably fast. As can be seen in Fig. 2.16, the energy in the rotation mode dissipated into modes with the higher eigenfrequencies, then as time passed, it transfers to the modes with 


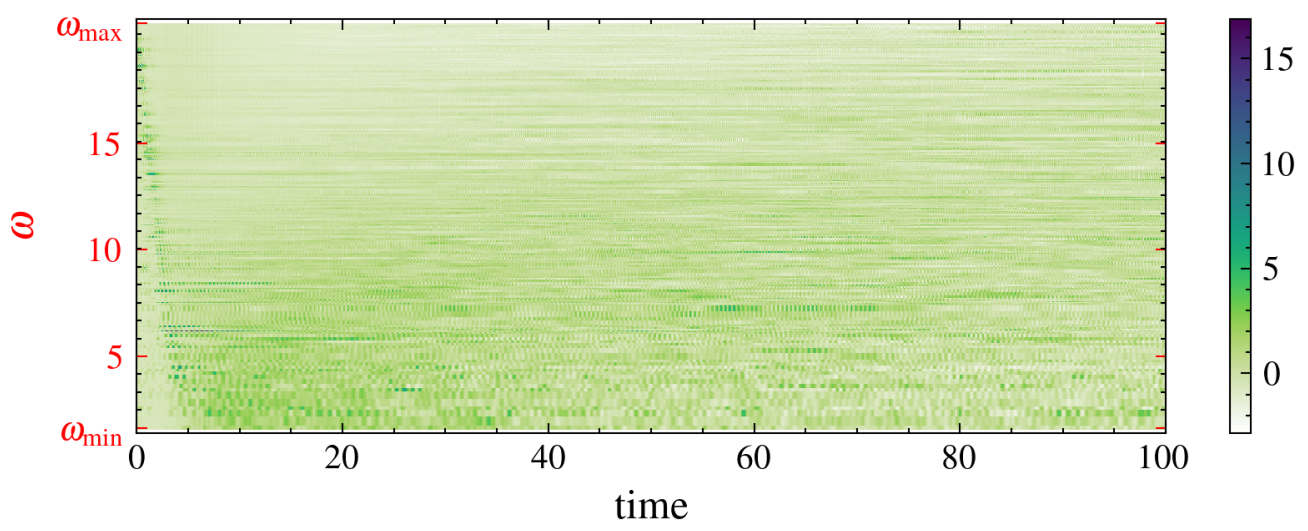

Figure 2.16: Heatmap of harmonic obc with $v_{\text {kick }}^{\text {rot }} \simeq 1.34$. The mode activity are shown with color-coding, and the illustration of the data is the same same before.

lower frequencies until the slider is thermalized. This dissipation of energy is extremely fast and system thermalizes in less then 10 time steps.

The extremely fast thermalization of this rotated obc slider seems to be a consequence of its rotational freedom. As we observed, when the kinetic energy was inserted into the rotational mode, it transferred faster to the other modes and increased the friction. The vibration of the breathing-like modes caused the slider to change its bond length; basically, the bond length increased as the system's temperature raised. This change in system size, alongside the rotational freedom, allows the system to find a commensurate structure with substrate and trap in it.

\subsubsection{Rigid edge}

In direct and rotational kicking, most active modes at the beginning and during decay have vibration that depend on the existence of edge. By making the springs between the particles on the edge extremely stiff. For (in other words, the edge is kept as a stiff body; still, we calculated the time-integrating for internal particles similar to the normal slider.), we looked at how the total behavior of the system was affected by it [App. A.1].

Direct kicking. Applying this change on the edge made the slider slide longer; besides, displacement in $y$-direction was increased. The slider's mean square displacement with the rigid edge was almost five times bigger than the slider with free edges when kicked with $v_{\text {kick }}=1.0$. The mode activity for this harmonic slider with the rigid edge kicked with $v_{\text {kick }}=1.0$ is shown 
in Fig. 2.17. One noticeable change in the modes actively is shifting from lower frequencies to the higher ones. Still, few modes with low frequencies got more active by this change. The modes that absorbed more energy and stored the energy for a while do not have edge vibrations. Moreover, some modes remained inactive during the simulation. These modes show vibration mostly on their edge, and the amplitude of the inner particles are very small.

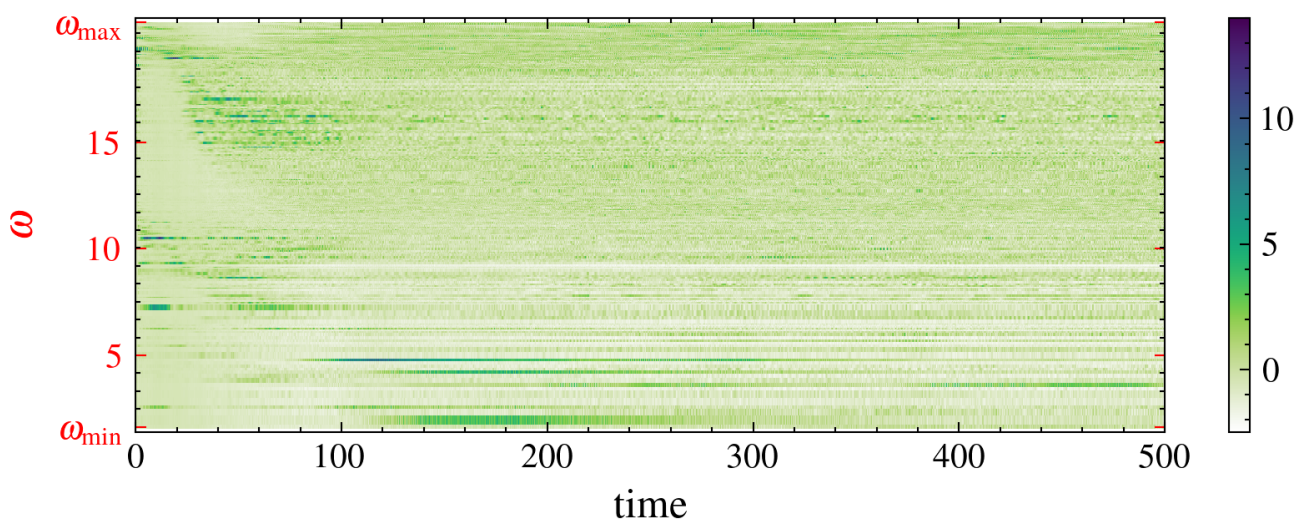

Figure 2.17: The heatmap of modes activity for the harmonic slider with rigid edges with $v_{\text {kick }}=1.0$. The mode activity are shown with color-coding, and the illustration of the data is the same as in Fig. 2.12

Rotational kicking. The changes that happened for the direct kicking could also be seen when we rotated the rigid slider with $v_{\text {kick }}^{\text {rot }} \simeq 1.34$. In this case, the slider showing an oscillation between counterclockwise and clockwise rotation. This oscillation was mainly a result of the activation of one mode. The activity of the particles on the edge of this mode is almost zero; also, the inner particles have complex rotational vibrations. This nondegenerate mode kept losing its energy and gaining most of it back until thermalization. In addition, the slider moved back and forth in both the $x$ and $y$-direction [Fig. A.6].

The slider with a rigid edge was more displaced mainly because it cannot expand and, while rotating, it was harder to find a commensurate structure with the substrate. Freedom of the particles that have bonds with the edge was also reduced, which decreased the slider's total degrees of freedom. This reduction of inner freedom decreased the intrinsic friction of the slider. 


\subsection{Other parameters}

For the sliders we studied here, we assumed Thai the slider and substrate were defect-free. Also, we presumed that both lattices had triangular structure, and they were incommensurate. Here we will briefly discuss what would happen if we changed one of the presumed conditions. First, we will look at different initial velocities and then examine how substrate conditions affect the slider frictional response. Moreover, we will look at defects in the interface and their effect on frictional behavior.

i) Initial velocity effect. We showed that the frictional behavior of the sliders strongly depended on the value of the initial kick. The velocities calculated from washboard frequency raise friction in the interface to the maximum in the pbc slider. We also studied the system's behavior with many different initial velocities for both harmonic and anharmonic sliders. When the slider is kicked, it slides and then thermalizes. After thermalization, the COM position of the slider hardly moves. Hence, we can look at the $\sqrt{\text { msd }}$ of a quantity to compare the effect of different $v_{\text {kick. }}$. The calculated $\sqrt{\mathrm{msd}}$ for the pbc slider is plotted in Fig. 2.18.

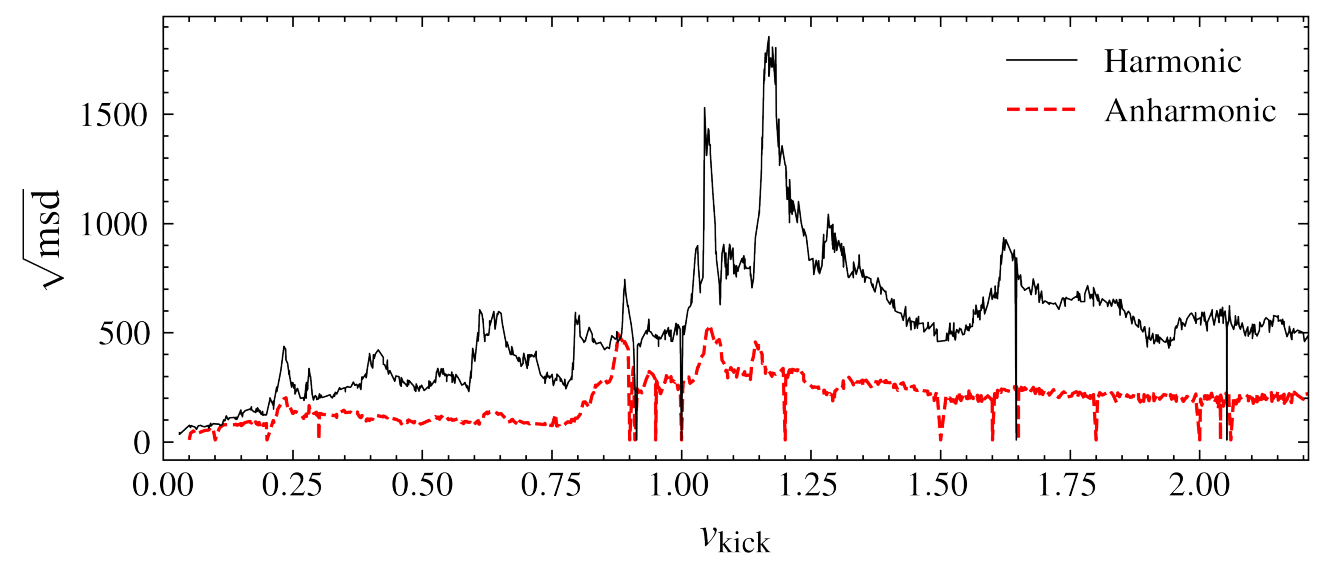

Figure 2.18: The figure shows $\sqrt{\mathrm{msd}}$ for the harmonic and anharmonic slider. The data was gathered for more than 1000 different velocities, which were selected based on different conditions (over 20 distinct trajectories of the slider were averaged to get these results, and each was initiated with a displaced position). The solid black curves show the $\sqrt{\mathrm{msd}}$ for the harmonic slider and red dashed curves for the anharmonic slider.

This figure displays that the three different velocities predicted by the washboard frequency have the highest friction for the harmonic slider. Still, 
there is another velocity with high friction $\left(v_{\text {kick }}=1.0\right)$, which did not predict by washboard frequency. The total trend of the harmonic slider demonstrated low friction for the velocities between the rang of $1.1 \lesssim v_{\text {kick }} \lesssim 1.3$, with a peak at $v_{\text {kick }}=1.168$ [cf. Fig. 2.9]. These high $\sqrt{\mathrm{msd}}$ values are mostly related to the initial transition of the slider. In these initial transitions, mainly few modes with high activity coupled together and remained active until thermalization [Fig. A.2]. The anharmonic slider, in general, slides for a shorter time than the harmonic slider. Although the anharmonic slider showed high friction at resonant velocity $\left(v_{\text {kick }}^{\star, i}\right)$, there were few more resonance with low $\sqrt{\mathrm{msd}}$. These results indicated that for a highly anharmonic slider, the anharmonicity of the bonds led to more resonances velocities. Another critical point is that the higher velocity does not necessarily mean higher $\sqrt{\mathrm{msd}}$. Moreover, Eq. 2.13 can fit all the curves with these velocities. The obc slider, however, did not show any irregular behaviors with different initial kicks. The harmonic and anharmonic sliders behaved similarly, and with increasing $v_{\text {kick }}$, the distances they slid were monotonically increased.

ii) Substrate density. For a defect-free substrate, besides its interaction strength with the slider, its geometrical properties such as lattice's type and periodicity can modulate the frictional behavior of the slider. For the harmonic slider, the periodicity of the lattice determined the resonance velocities. However, it is not the only effect of this property. The substrate also has a critical impact on the dominant modes during sliding of the sliders. The effect of commensuration ratio on friction between moving bodies is well known. The results for the pbc slider we presented here had the commensuration ratio $\rho=a_{b} / a_{s} \sim 1.5714$, and as we saw, there were a few dominant modes. Our simulations showed that the most active modes for a system with slightly different commensuration ratios were entirely different. For instance, when $\rho=1.2857$, the most dominant modes immediately after kicking were those with frequencies equal to 9.2, 14.2, and 20.2 [cf. Fig. A.7]. Still, the initial kick will affect which of those modes reserves more energy.

In the obc slider, the anharmonicity of the system suppresses this geometrical property of the substrate. With increasing (decreasing) the interaction strength as a result of the higher (lower) density potential friction in the interface increased (reduced).

iii) Substrate's structural disorder. Our investigation on the effect of the substrate's structural disorder (dislocation of the potential sources) on the frictional behavior of the slider showed a significant relation. To study this effect, we kept the slider intact: a full periodic harmonic slider with 
$N=196$ particles and bond length equal to one. First, we displaced only one potential source, which was selected randomly. To ensure that the displaced source did not overlap with other sources, it gets a random displacement between zero and half of the lattice's constants (i.e. $\frac{7 / 11}{2}$ ) in both $x$ and $y$ direction ${ }^{6}$. As an impact of this dislocation slider with different $v_{\text {kick }}$, its $v_{\mathrm{COM}}$ decays faster, and the system thermalizes rapidly. Also, the mode activity changed. For instance, when $v_{\text {kick }}=0.05$, all the modes were activated even though there were still a few dominant modes, but other modes adsorbed energy too. With $v_{\text {kick }}=1.168$, the effect is more visible. Its $|\bar{\mu}|$ (i.e. the absolute value of COF) increased from 0.1 to 0.66 and $c$ reduced from 1190 to 230 [Tab. 2.1]; also, the lifetime of the most active mode decreased.

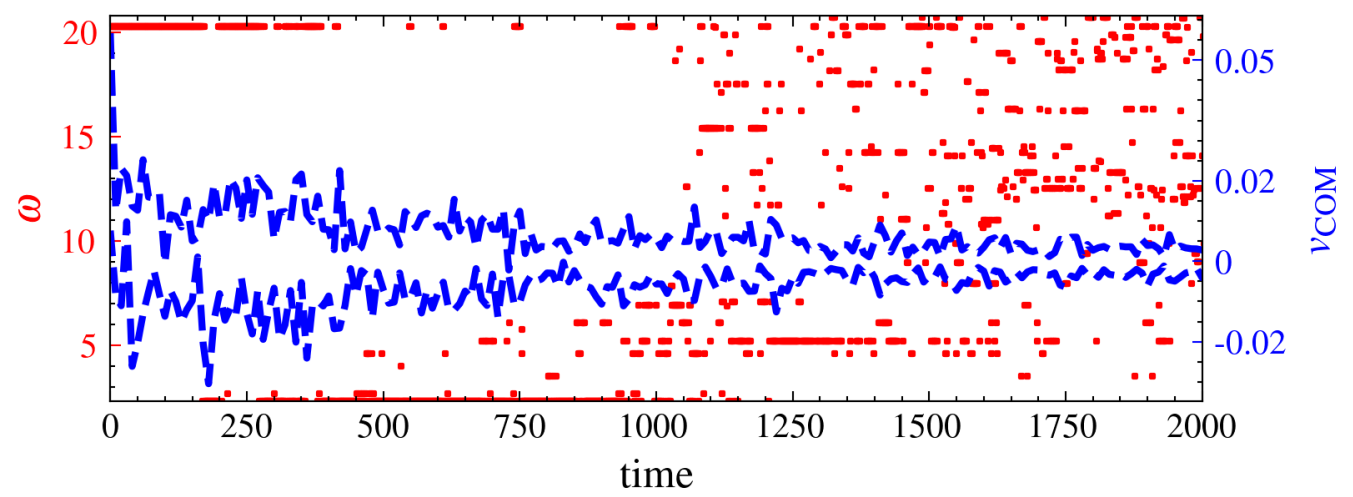

Figure 2.19: Slider with periodic boundary conditions with a fraction $2 \%$ of randomly displaced potential sources in the substrate, at kick velocity $v_{\text {kick }}=0.05$. (over 20 distinct trajectories of the slider were averaged to get these results, and each was initiated with a displaced position and also different random defects in the substrate).

Moreover, we looked at the substrate with more dislocated sources: $2 \%$ were randomly selected and displaced like single displacement. The most active modes for $v_{\text {kick }}=0.05$ now are ones with lower frequencies $\left(\omega_{i}<6\right)$. Interestingly, with this amount of defects and also for single dislocation, the most active mode is the mode with resonance eigenfrequency $\omega^{\star}=20.26$. The result for this velocity is shown in Fig. 2.19. For the $v_{\text {kick }}=1.168$, with $2 \%$ defect sliders decays quickly and thermalize extremely fast, with $|\bar{\mu}|=1.72$ and $c=78$. When the substrate lost its periodicity completely (amorphous structure), the slider started to thermalize immediately and hardly moved.

\footnotetext{
${ }^{6}$ In the pbc slider, there is a possible artificial effect due to periodic boundary conditions. However, since the potential source was static and there was no interaction between them, we can ignore these effects [247].
} 
iv) Substrate's lattice type. When we used a different substrate's lattice type, other slider modes got active during sliding. For example, when the substrate had a tetragonal lattice, the modes with the most energy had low frequencies, specifically $5 \lesssim \omega \lesssim 6$. We used the pbc slider with 196 particles, unit mass $m=1$, and bond length equal to 1 to study this impact. Although the ratio between the number of particles in the slider and the number of substrate potential sources is different from the hexagonal substrate, it gave an insight into the relation between interface friction and geometrical properties of the two surfaces.

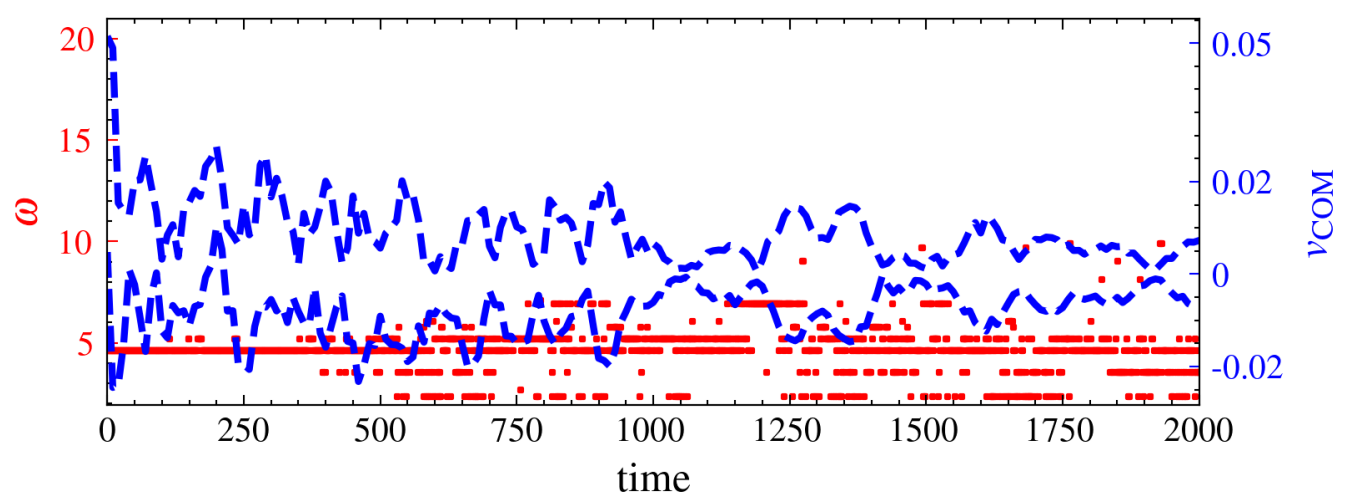

Figure 2.20: Fully periodic slider on a tetragonal substrate, at kick velocity $v_{\text {kick }}=0.05$. (over 20 distinct trajectories of the slider were averaged to get these results, and each was initiated with a displaced position).

Fig. 2.20 shows the sliding behavior of the pbc slider using $v_{\text {kick }}=0.05$. Besides the fast decay of $v_{\mathrm{COM}}$, the most active modes are interesting. They are showing how substrate periodicity can affect the shape of dominant modes. The most active modes displacements are mainly in the $x$ - and $y$-direction. The same behavior also can be seen for the obc slider.

v) Slider with defects. One can also investigate the consequence of missing bond on the frictional behavior of the slider. To see the effects of this defects, we used the pbc slider (slider contains $N=196$ particles, each with unit mass $m=1$ ), the only change is we cut a $2 \%$ of randomly chosen springs (for this small number, the slider is still a unique system and there are no separated particles free in the slider). The potential surface also remains as before, i.e. with complete crystalline structure. Fig. 2.21 illustrate the sliding behavior for this defected slider, having harmonic interactions and kicked by $v_{\text {kick }}=0.05$, that can be compared with the intact slider of Fig. 2.12. 


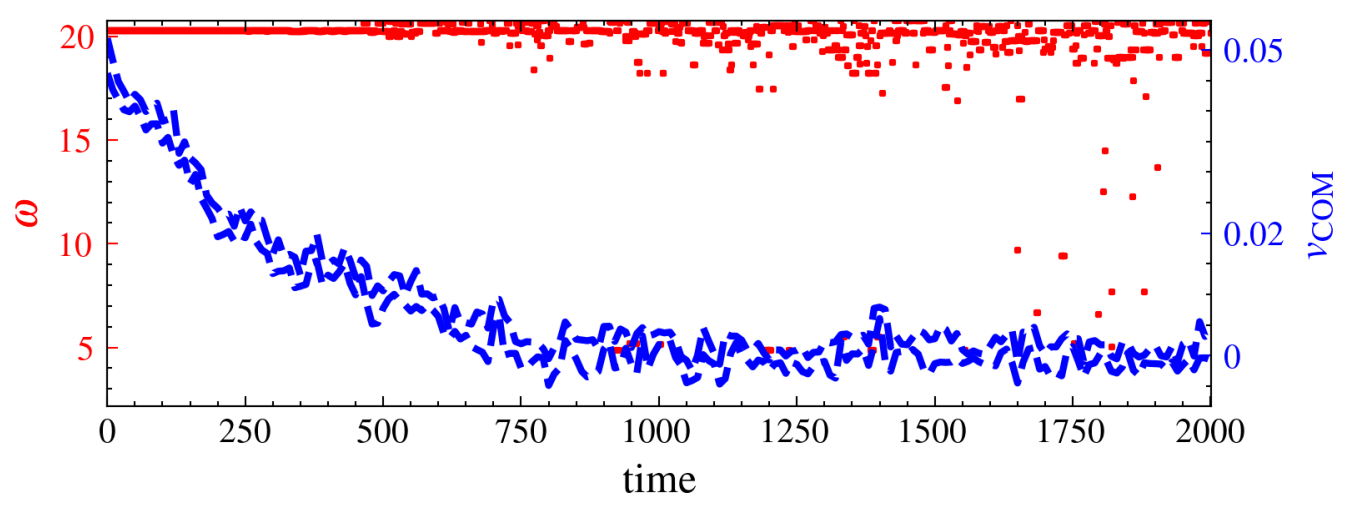

Figure 2.21: pbc slider with a $2 \%$ cutted springs which are randomly chosen, at kick velocity $v_{\text {kick }}=0.05$. (over 20 distinct trajectories of the slider were averaged to get these results, and each was initiated with a displaced position).

The results illustrated that the difference is noticeable. A tiny fraction of defects significantly increase the thermalization rate, indicating much higher friction, while the intact slider did not thermalize at all. Mode behavior at short time intervals is still focused on the main mode $k^{\star}$. The existence of only a tiny fraction of defects is enough to demolish the coupling with $k^{\star}$.

vi) Commensurate interface. It is well known that dry friction between surfaces in a commensurate interface is higher than in an incommensurate interface. In the real world, commensurate and incommensurate states often exist simultaneously, also there is transition between commensurate to incommensurate phases $[183,248,249]$. This transition is usually described in terms of kink, wall, or soliton formation. In experiments such as epitaxy, one condition for commensurability between deposited film and substrate is the equality of the in-plane lattice spacing at the interface $[59,250]$. It is also common to describe commensurability as a "coverage parameter" at the ground state (i.e. the atom configuration with the lowest potential energy). In this situation, the "commensuration array of particles" define when each minimum of the substrate potential is occupied by one particle. By this definition, the commensurate state is when the number of particles equals the potential wells' numbers [34, Chapters 1 and 5] or their ratio is a simple rational fraction [248].

In the systems we studied, the incommensurability was introduced to the interface by setting the ratio between surfaces lattices' constants $(\rho)$ as golden ratio. If we put this ratio to $\rho=1$, we would have a perfect commensu- 
rate interface. In such a condition, friction was significantly higher in both pbc and obc sliders. Still, we can introduce incommensurability to the interface by rotating the slider on its $x y$-plane on top of the substrate [251, 252]. It has been shown that a slight misalignment can lead to significant changes in the system. For example, the term "Magic-Angle" [84, 253-258] has been used widely in describing unique properties (such as superconductivity) of the twisted double layer of graphenes [259, 260].

We also studied the frictional behavior of commensurate sliders and in more detail for the obc slider. The friction, in all cases, was higher compared to the incommensurate sliders. The importance of commensuration of the lattices is more evident when we consider that the number of potential sources in the commensurate interface was lower than the incommensurate interface we studied. The results showed that even though the total potential from the lattice reduced but friction increased. The role of rotation was very evident in these conditions. Slider rotations were nearly connected with translations. However, as soon as the slider-substrate orientations matched, the center of mass translational energy displayed a sudden decline with an increase of the slider angular speed, afterwards reversing back to transnational [165]. This translation-rotation exchange was highly dependent on the strength between particles in the slider; as much as this interaction was strong, this behavior was more prominent. The orientation of the slider on the substrate before kicking can significantly impact the friction, even for a small misalignment angle. When we rotated the slider with $21.79^{\circ}$ counterclockwise (the most commensurate state in the rotated interface), friction was the highest. Moreover, the friction was the lowest when it rotated to $30.0^{\circ}$ which was the most incommensurate state in the rotated interface ${ }^{7}$.

\footnotetext{
${ }^{7}$ These angles are calculated from the moiré pattern relations [59, 250]. The moiré pattern and its importance in studying and modifying the interface's properties have been the subject of many investigations [60, 95, 100, 168, 261-266]. The method of calculations and results for this part are not presented in this thesis.
} 


\section{Chapter 3}

\section{Conclusions}

In this work, the frictional behavior in the relative motion of a dry interface was studied. This interface was studied based on the assumption that in the absence of electronic excitations and chemical potentials, the excitation of lattice vibrations causes the dissipation of energy. It is supposed that one layer was rigid (acting as a simple cosine potential surface with lattice constant $a_{b}$ ) and friction in the interface occurred due to the lattice vibrations of the slider. The slider was set into motion by an initial kick. Also, it is assumed that the vibrational motion initiated by the initial velocity leads to the dissipation of energy. In dry friction, this dissipation is exactly the process by which the motion of the slider is converted into vibrational (i.e., thermal) energy. In this regard, studying a slider with an many number of atoms (i.e. with periodic boundary conditions) is essential to understand how the inserted kinetic energy by initial kicking is dissipated among the vibrational modes of the slider. Therefore, the slider with periodic boundary conditions (pbc) on an infinite substrate was studied. The results of this system gave an insight into behavior of a more realistic model: a slider with edge defect, i.e., the slider with open (free-end) boundary conditions. In the free-end boundary conditions, there are no external forces on the edges of the slider. These are the most straightforward boundary conditions that allow the slider to expand and contract freely under the effect of the substrate potential $\{127,182,183,188,214,249,267,268\}^{1}$.

In the designed system, the slider and substrate both had lattices with triangular symmetry. The two lattices were incommensurate, whereas the commensuration ratio between lattice constants was set equal to the golden

\footnotetext{
${ }^{1}$ The assumptions in this work are based on the references, which are indicated by curly brackets \{\} .
} 
ratio. Also, the Frenkel-Kontorova model was applied at zero-temperature and in an unpinned state (below the Aubry transition). Furthermore, molecular dynamics simulations were employed for this work, which has been shown to be suitable for studying friction. In this study, the main focus was to analyze the vibrational modes of the slider while interacting with a potential barrier with no thermostat and possible artifacts of the thermostat on the frictional behavior. The simulations started with a condition far from equilibrium (i.e., the slider was not optimized) to observe the process of "mode mixing" and "thermalization" in the slider. The vibrational modes of the slider are computed from the interparticle potential and the particle masses, where the vibrational eigenfrequency (eigenvalue) and mode unique shape (eigenvector) depend on the second spatial derivativation of the system's potential energy with respect to the point-like particles displacements around the ground state. Interactions between vibrational modes that enable them to interchange energy which is depend on the potential's higher-order derivatives (anharmonic terms). Prior to each sliding simulation, the slider received a global random displacement on the substrate to probe possible effects of the precise initial condition $\{34,134,180,182,183,188,210,225,225,269-274\}$.

In the studied system, the internal energy was due primarily to the lattice vibrations of the slider $\{73\}$. When the kinetic energy was put into the slider (by initial velocity), the first eigenmode, $k=0$, which has zero frequency (one of the goldstone modes [92, 228]) first adsorbed this energy. Of course, the inserted energy should be sufficient to overcome the potential barrier arising from the substrate in order to displace the slider, but since we are below the Aubry transition, this is always the case $\{275\}$. It has been shown that in combination with nonlinearity, the modes with low frequency can also grasp energy quickly $\{153\}$. The coupling between $x$ and $y$ coordinates (one source of nonlinearity) of the slider in the interaction with the substrate does lead to some damping of the vibration modes (phonons). This "damping is anharmonicity of the lattice, which causes the vibrational energy of one mode to transfer to the other modes" [73]. Hence, modes that are not purely polarized in one direction get activated. If this damping constant is small, for example, in a small off-resonance kick, the energy from fist mode transfers and remains in a few (a group of) modes and does not transfer to other modes. In other words, in a comparatively brief time, "the energy, which was initially concentrated in a few modes, is spread among a larger group of them, creating a 'packet' of naturally excited modes" [273]. This situation is metastable, and the system evolves towards equipartitioning on a long time scale. This is a well-known problem studied by Fermi, Pasta, Ulam, 
and Tsingou (FPUT) [276-278]. In their study, energy was at first put into one eigenmode of a model solid (a vibrating string) with a small amount of anharmonicity and fixed boundary conditions. Contrary to expectation based on the equipartition principle, the energy did not distributed between the other modes of the slider. Later, it was shown that the anharmonicity in the FPUT system is too weak for the system to be chaotic, and hence it is nonergodic [73]. It has also been shown that for the 2D FPUT model, the time scale where the initial energy is shared among only a small fraction of eigenmodes is extremely short, compared to the 1D model [273, 274].

These consequences of anharmonicity were observed in our results. In addition to the coupling between $x$ and $y$ components of the particles' position, anharmonicity could be introduced into the interface from the particleparticle interactions. The existence of defects in the interface also strongly raises the anharmonicity. Whenever the anharmonicity is high, irrespective of its source, the decay of the center of mass velocity is fast. Another result of strong anharmonicity in the defect-free pbc slider is that it had a range of resonances $\{73\}$, which led to drastically high friction. The numbers of resonances in the fully periodic slider with anharmonic interaction are higher than in the harmonic slider. This higher anharmonicity indeed causes the system to have higher friction. By adding defects into the interface, it was observed that friction increased as the ratio of the defects increased, resulting from risen anharmonicity in the interface. It has also been shown that the randomly distributed point defects always give rise to pinning [247, 268, 279 281]. If the damping is strong enough, the energy spreads quickly to all the vibrational modes, i.e., the slider exhibits ergodic behavior; as a result, the slider thermalizes rapidly, and friction is high.

For the slider with free-end boundary conditions (obc), energy from the kick easily transfers to the pure rotation mode $(k=2)$. Quickly afterwards, it transferred to the modes that have polarization along the lattice's diagonal (breathing-like vibrations $\{153\}$ ). The freedom of rotation for this slider opened another channel for dissipation. This rotation also allows the modes with twisting-and-torsion vibration $\{241\}$ to gain energy. Alongside these vibrations, particles on edges play a crucial role in total friction. Due to their low restriction, the edge bonds are more flexible than those located within the contact area, allowing them to have relatively high activities, thus, they may undergo more significant distortions during sliding. Since the vibrations of these particles have a substantial effect on high friction for this slider, the edge acted as pinning sites during sliding, accelerated energy dissipation. At 
the time that energy from translational modes transferred to the pure rotational mode, which also has zero frequency, energy transformation to other modes happens faster. One outcome is that the existence of this duration of the lattice vibrations plays an essential role in the velocity dependence of the force of friction for any finite-size slider $\{183\}$. These results of edge-effect on the frictional behavior were also observed in other studies such as Refs. $[38,86,92,92,95,282,283]$.

When the obc slider is rotated with an initial kick instead of direct kicking, the energy is inserted into the third mode $(k=2)$. In this way, the thermalization is extremely fast, demonstrating the importance of free rotation on the higher friction for the slider with free-end boundaries. Another aspect of this slider behavior is that when the slider got a direct kick $\left(v_{\text {kick }}=1.0\right)$, it expanded, increasing the length of bonds by about 25\%. This change happens after energy is transferred to other modes and when the slider is close to thermalization $(t \simeq 800)$. This expansion is mainly a result of the activation of modes with breathing-like vibrations. When the slider kicked with rotational velocity $\left(v_{\text {kick }}^{\text {rot }} \simeq 1.34\right.$ ), this expansion is not only broader (about 30\% expansion) but happens considerably faster $(t \simeq 20)$. This rotation and expansion allowed the slider to find a perfect registry and stay in it; in other words, "locked together via local regions of common periodicity" (commensurate structure) as mentioned by the Refs. [85, 92, 284].

The edge is an intrinsic "defect" that every system with free-end boundaries contains [94, 282]. By making the bonds on edges rigid, energy dissipation among modes was entirely modified. This modification blocked the transformation of energy to the modes with high vibration on the edge. It also eliminates the slider from expansion and stops the twisting and torsion vibration of the edge particles. These limitations made the energy transfer to the different modes whose primary vibrations do not involve edge activities. In addition, the slider did not expand, and friction was reduced with the diminished activity of torsion modes. Besides that, the directly kicked slider with rigid edge moved further; and rotational kicking energy transformed back and forth to the translational modes, making the slider show diffusive behavior $[165,168,241]$ on the substrate. As the number of rigid bonds in the slider increased, this behavior of the slider became more visible ${ }^{2}$. This is reasonable; when a fully rigid slider is kicked on the substrate, only the three goldstone modes are available for the slider; and the exchange of energy between these modes causes a random walk behavior in the slider.

\footnotetext{
${ }^{2}$ The results for the rigid slider were not reported in this thesis.
} 
The effect of surface dislocation on the friction in the interface is also discussed in this study. Moreover, the results contended that the anharmonicity of the lattice is the source of the resonances of friction. Still, the periodicity of the substrate determined the place where these resonances happened. The relationship between the frictional behavior of the pbc slider and the periodicity of the substrate can be seen through the washboard frequency relation [285]. In addition to indicating the resonance velocities, this periodicity could also affect the most active modes. For the same slider, most active modes resembled the pattern of the superstructure made of the slider and substrate. When the substrates have different lattice types (e.g. simple tetragonal) with a low kick to the slider, the activated modes have polarization analogous to the substrate's periodicity ${ }^{3}$.

In summary, we have conducted the classical MD simulations and eigenmode analysis to investigate the phonon energy dissipation in a friction process of a kicked slider. Once the slider receives initial energy, the dissipation of energy starts and goes on until the slider thermalizes; the path of thermalization depends on one key parameter of the interface: anharmonicity. The anharmonicity could be intrinsic, e.g. type of the bonds or existence of the edges, or extrinsic like defects in the interface, or from geometrical properties of the substrate. Strong anharmonicity can also suppress the structural lubricity from incommensurability of the interface. This classical harmonic model is very successful in describing the modal activity of the slider. Extending this model to three-dimensions will be an interesting subject for further studies. We remark that the real nature of the complex processes happening at the interface of two interacting surfaces in relative motion is somewhat oversimplified in this model. Like every model, our model also has its limitations. In the case of the disorder in the slider, the critical assumption that all normal modes (phonons) have plane waves with explicit velocities is no more reliable. As it has been shown in the presence of a few percent of impurity concentration in the interface, the phonons attributes are drastically altered [198, 287, 288]. Hence, understanding the theoretical background is always essential to determine which features deserve consideration and which details are irrelevant. Choosing the most appropriate investigation approach is essential to elucidate the obscurity of the problem under study by considering the system's desired aspects [28, 38, 198].

\footnotetext{
${ }^{3}$ For an example study on the importance of the substrate shape on the atomic friction, see Ref. [286].
} 


\section{Appendix A}

\section{Supplementary information}

\section{A.1 MD implementation details}

The 2D model were implemented in LAMMPS [216]. All data were obtained in the microcanonical ensemble ( $\mathrm{fix}$ nve, timestep 0.001). For the rigid flake, fix rigid/nve was used to implement the rigid edge. The actual simulation output is the trajectory, which could include positions and velocities of the particles as a function of the simulation time. Having these quantities, one can compute the other wanted properties. An exclusive C-code based on the LAPACK package was used to compute the hessian and eigenmodes and analyze the MD trajectories to determine the mode kinetic energy and amplitude.

\section{A.2 Logistic regression}

In probability theory and statistics, logistic distribution is a distribution resembling the normal distribution with heavier tails. The cumulative distribution function (CDF) of the logistic distribution is an $S$-shaped curve known as logistic function. CDF of a multivariate variable $X$ distribution function expresses the probability that random variable $X$ takes the value smaller than or equal to $x$. CDF can also be expressed as the integral of the probability density function $f(x)$ [289, Chapter 6].

The general form probability density function for its distribution for variables $x$ is:

$$
f(x ; \mu, s)=\frac{e^{-(x-\mu) / s}}{s\left(1+e^{-(x-\mu) / s}\right)^{2}}
$$

where $\mu$ is the mean (and mode, and median), and $s$ is the scale parameter related to the standard deviation of the distribution [290, Chapter 23]. CDF 

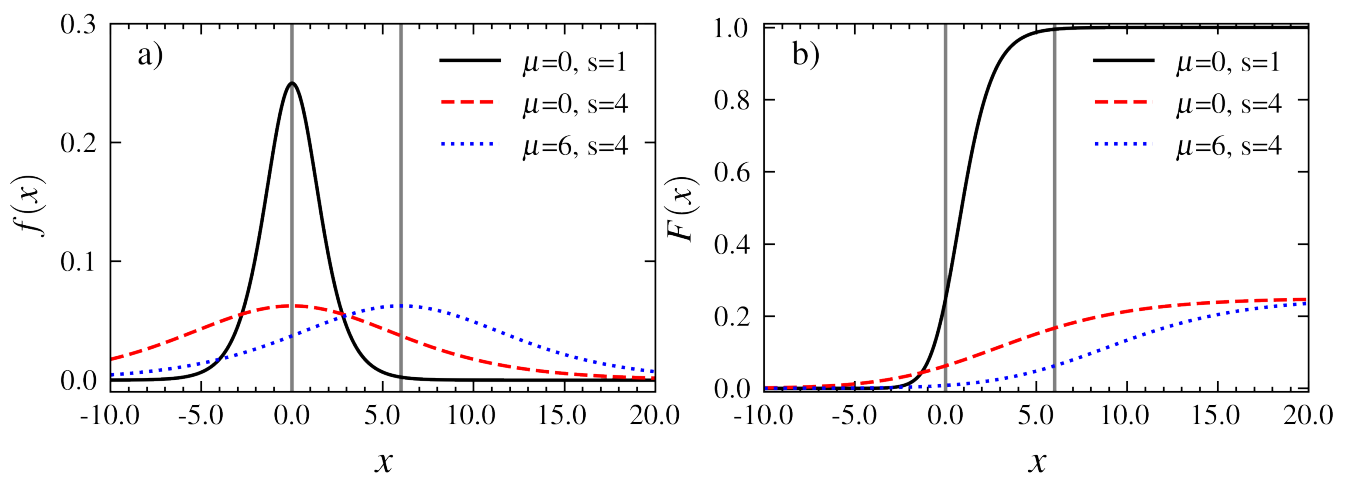

Figure A.1: Probability density function (a) and the cumulative distribution function (b) of the logistic distribution

equation determined from integral of distribution function [289, Chapter 6]. $\mathrm{CDF}$ of logistic distribution is:

$$
F(x ; \mu, s)=\frac{1}{1+e^{-(x-\mu) / s}}
$$

\section{A.3 Additional figures}

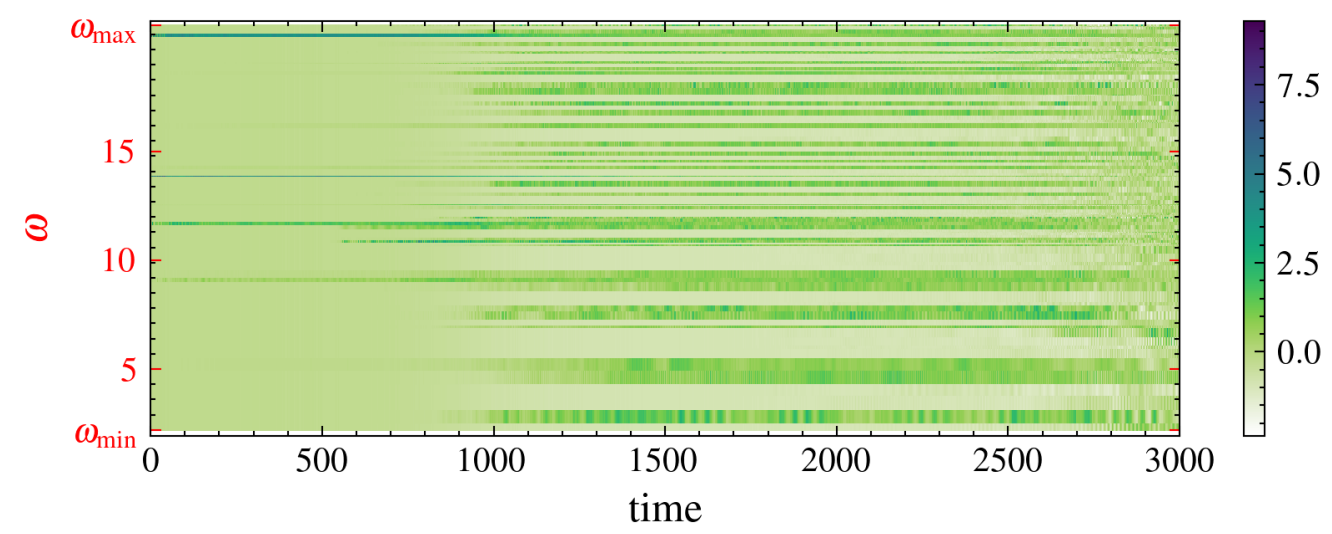

Figure A.2: Heatmap of harmonic obc with $v_{\text {kick }}=1.16$. The color-coding indicates the mode activity, the standard score of modes at each time step. (over 20 distinct trajectories of the slider were averaged to get these results, and each was initiated with a displaced position). 
a)

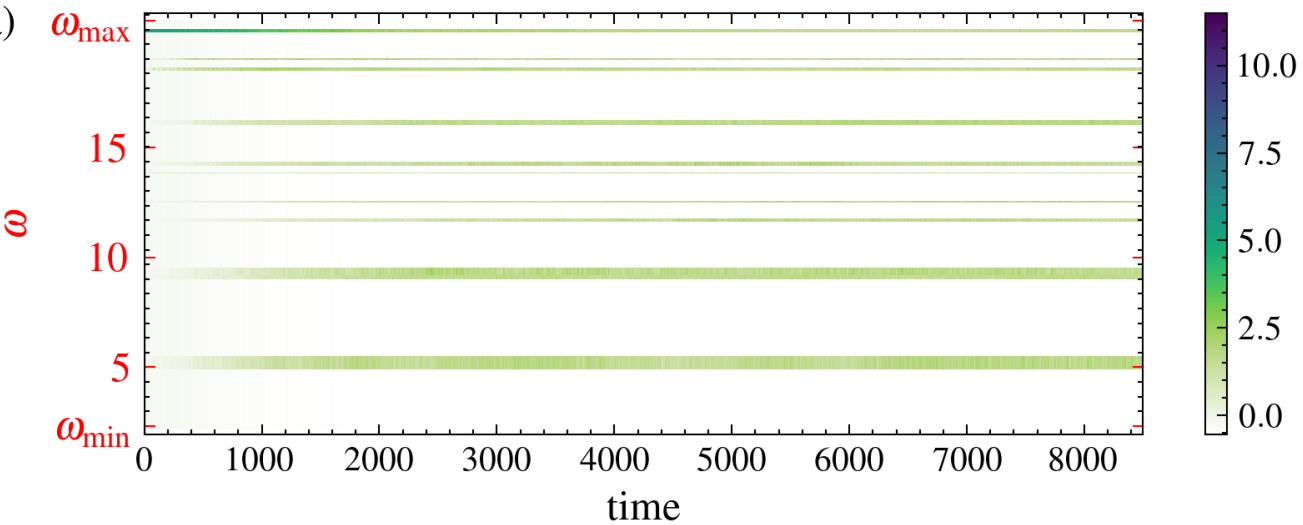

b)
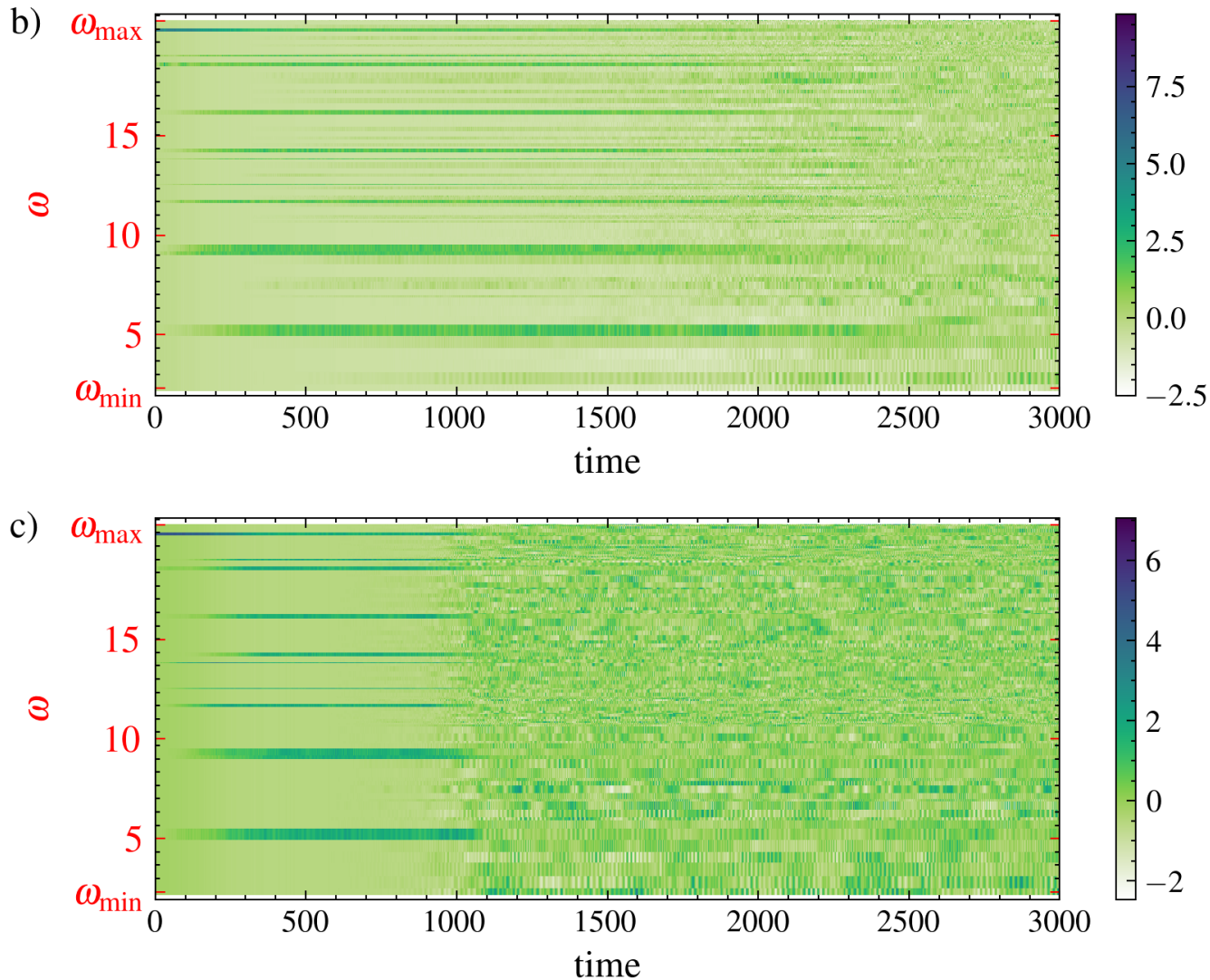

Figure A.3: Heatmap of harmonic pbc(Fig. 2.5). In (a) and (b) $v_{\text {kick }}=0.05$ and bonds are harmonic and anharmonic, respectively. and in (c) $v_{\text {kick }}=$ $v_{\text {kick }}^{\star}=2.052$ (first resonance velocity). The color-coding indicates the mode activity, the standard score of modes at each time step (over 20 distinct trajectories of the slider were averaged to get these results, and each was initiated with a displaced position). 


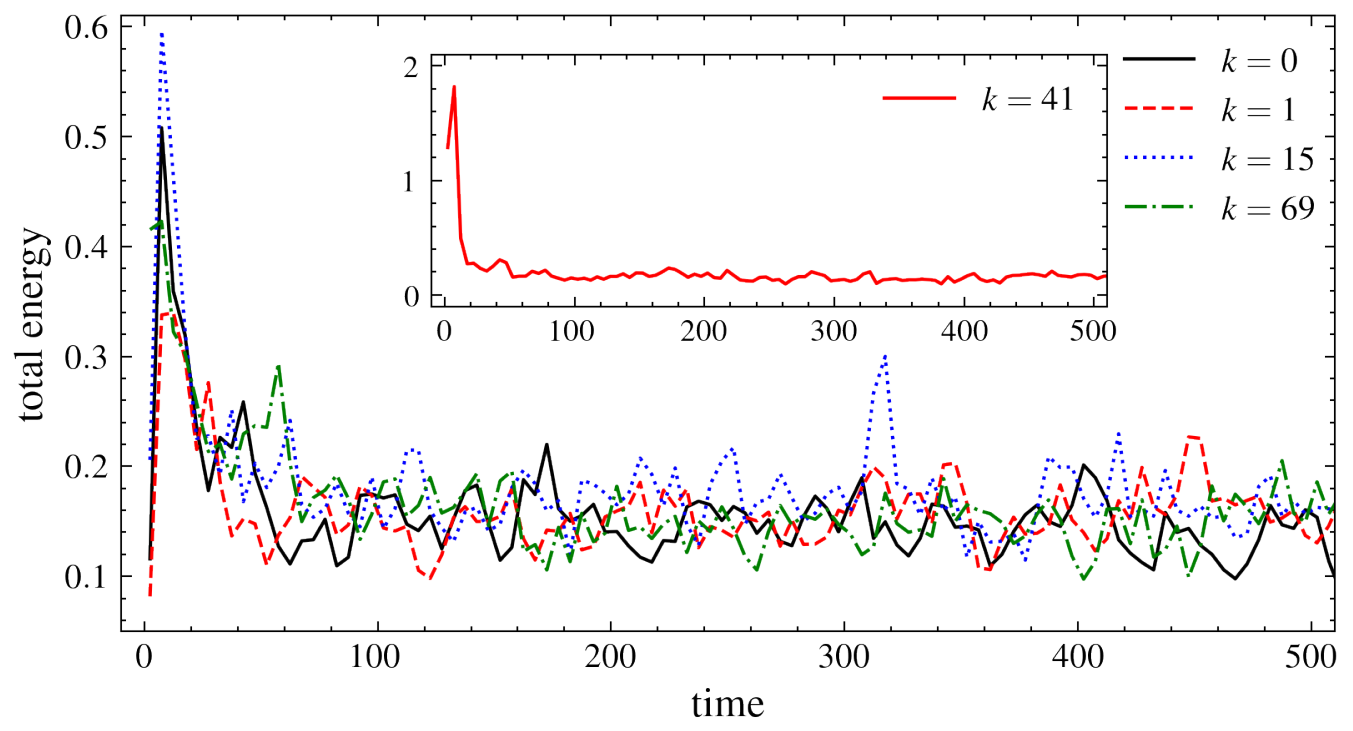

Figure A.4: Energy of six most active modes for the rotated slider with $v_{\text {kick }} \simeq 1.34$.

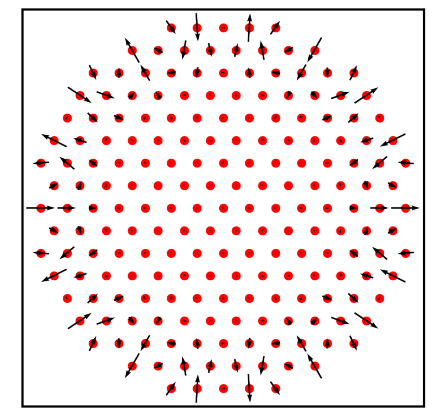

(a) $\vec{\xi}_{41}$

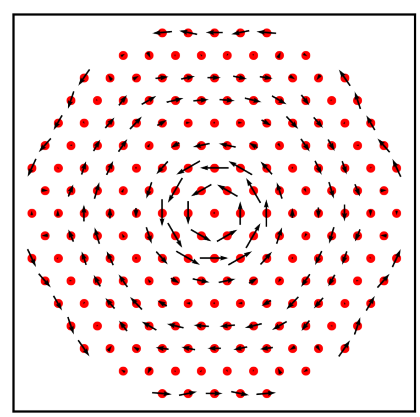

(b) $\vec{\xi}_{35}$

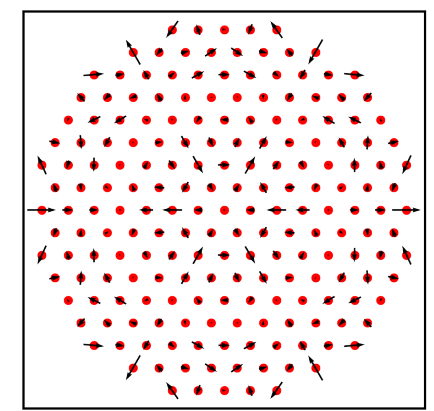

(c) $\vec{\xi}_{69}$

Figure A.5: The figure shows three of the most active vibrational modes of the rotated harmonic obc slider (Basically, the eigenvectors of the slider). The arrows indicate the amplitude and direction of the vibrations (i.e., mode polarization vectors). The index numbers are arbitrary; we ordered them based on their eigenfrequencies, $i=0,1, \ldots, 2 N-1$, and $N$ is the number of particles in the slider; a smaller number means a lower frequency. (a) shows a mode with very strong torsion activity, $\omega_{35} \simeq 5.57$. (b) is a mode with high vibration activity on edge $\omega_{41} \simeq 6.08$. mode in (c) is one modes with twistingand-torsion behavior and strong breathing-like vibration $\left(\omega_{69} \simeq 7.95\right)$. The motion for these modes is indicated with respect to the center of the slider. 


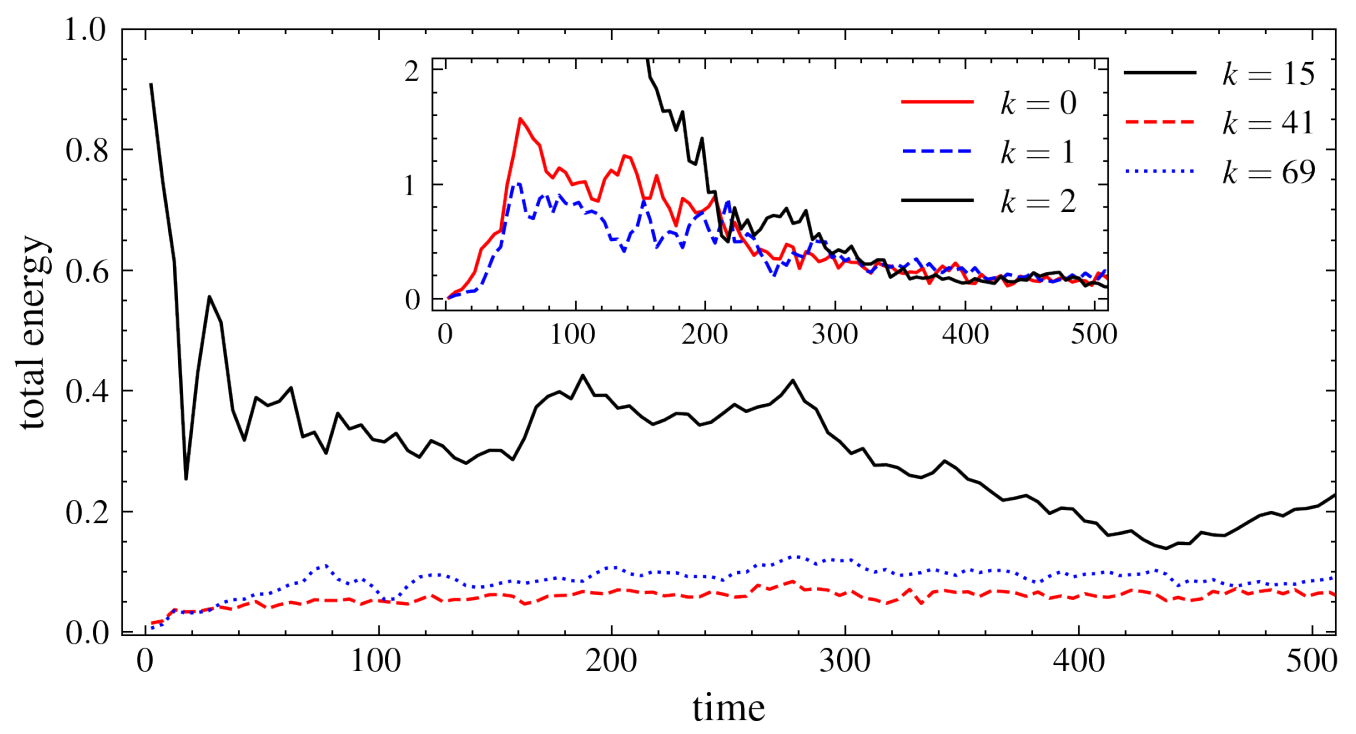

Figure A.6: Energy of six most active modes for the rotated slider with rigid edge with $v_{\text {kick }} \simeq 1.34$.

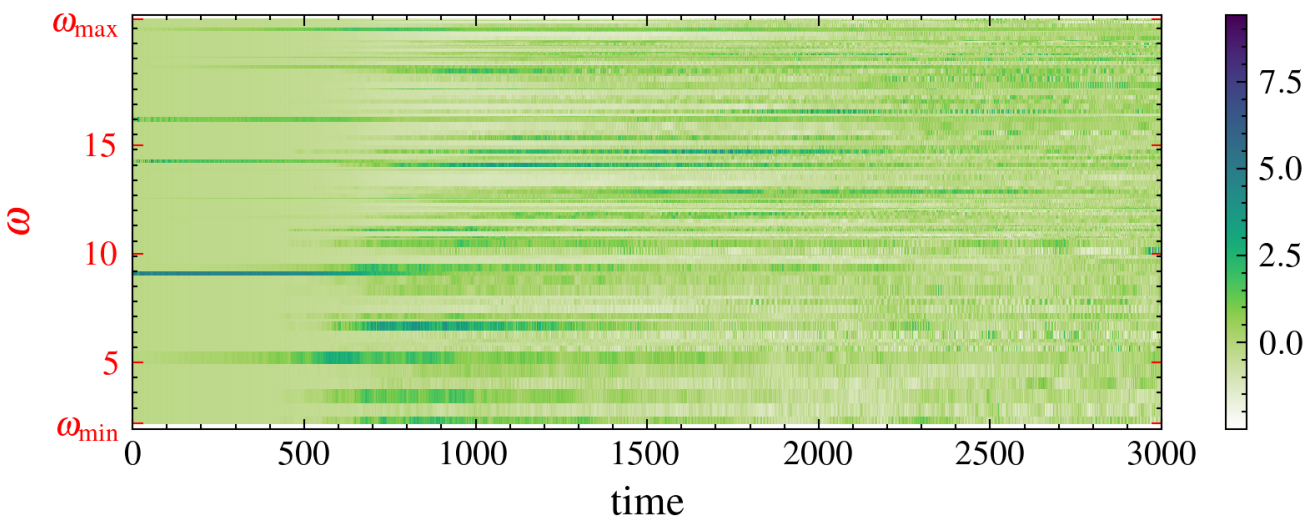

Figure A.7: Heatmap of harmonic obc with $v_{\text {kick }}=1.16$. The color-coding indicates the mode activity, the standard score of modes at each time step. with lattices' parameters ratio equal to 1.21 (over 20 distinct trajectories of the slider were averaged to get these results, and each was initiated with a displaced position). 


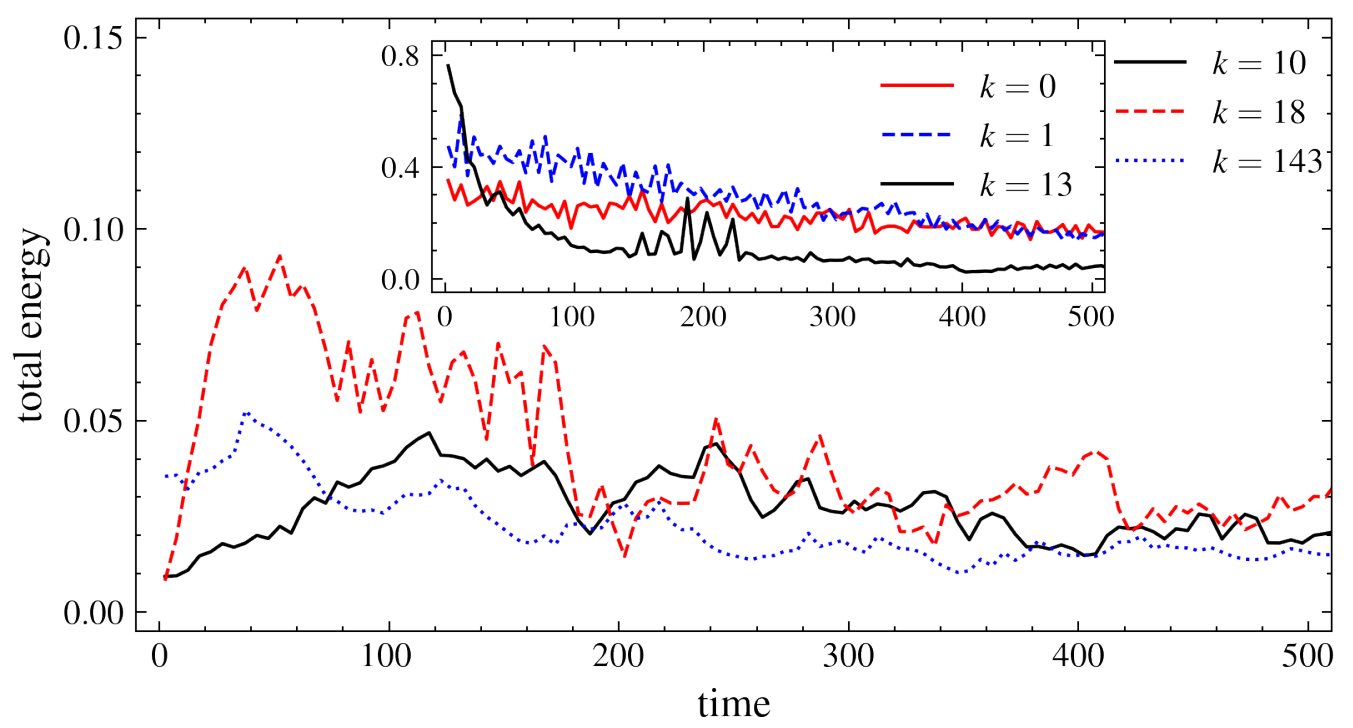

Figure A.8: Energy of six active modes for the pbc slider with tetragonal substrate with $v_{\text {kick }}=0.05$.

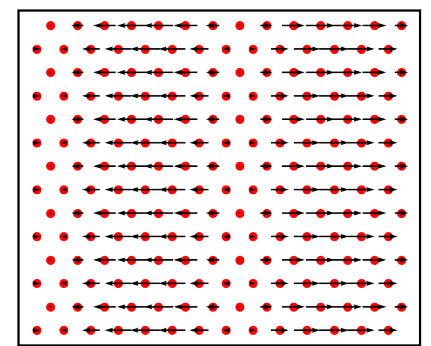

(a) $\vec{\xi}_{10}$

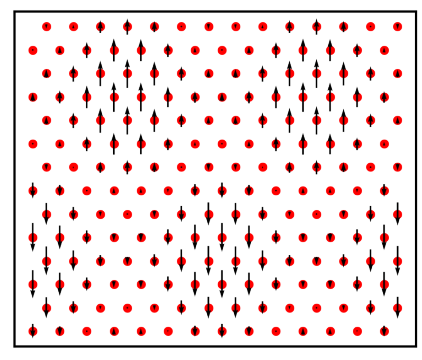

(b) $\vec{\xi}_{13}$

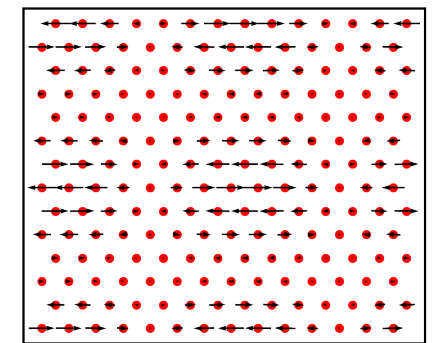

(c) $\vec{\xi}_{143}$

Figure A.9: The figure shows three of the most active vibrational modes of the rotated harmonic pbc slider (the eigenvectors of the slider) with $v_{\text {kick }}=0.05$. The arrows indicate the amplitude and direction of the vibrations (i.e., mode polarization vectors). The index numbers are arbitrary; we ordered them based on their eigenfrequencies, $i=0,1, \ldots, 2 N-1$, and $N$ is the number of particles in the slider; a smaller number means a lower frequency. (a) shows a mode with very strong torsion activity, $\omega_{10} \simeq 2.48$. (b) is a mode with high vibration activity on edge $\omega_{13} \simeq 2.76$. mode in (c) is one modes with twistingand-torsion behavior and strong breathing-like vibration $\left(\omega_{143} \simeq 13.25\right)$. The motion for these modes is indicated with respect to the center of the slider. 


\section{Appendix B \\ Reprints permissions}




\begin{tabular}{|c|c|}
\hline \multirow{6}{*}{ स्बु ACS Publications } & Superlubric Sliding of Graphene Nanoflakes on Graphene \\
\hline & Author: Xiaofeng Feng, Sangku Kwon, Jeong Young Park, et al \\
\hline & Publication: ACS Nano \\
\hline & Publisher: American Chemical Society \\
\hline & Date: Feb 1, 2013 \\
\hline & Copyright $\odot$ 2013, American Chemical Society \\
\hline
\end{tabular}

\section{PERMISSION/LICENSE IS GRANTED FOR YOUR ORDER AT NO CHARGE}

This type of permission/license, instead of the standard Terms \& Conditions, is sent to you because no fee is being charged for your order. Please note the following:

- Permission is granted for your request in both print and electronic formats, and translations.

- If figures and/or tables were requested, they may be adapted or used in part.

- Please print this page for your records and send a copy of it to your publisher/graduate school.

- Appropriate credit for the requested material should be given as follows: "Reprinted (adapted) with permission from (COMPLETE REFERENCE CITATION). Copyright (YEAR) American Chemical Society." Insert appropriate information in place of the capitalized words.

- One-time permission is granted only for the use specified in your request. No additional uses are granted (such as derivative works or other editions). For any other uses, please submit a new request.

If credit is given to another source for the material you requested, permission must be obtained from that source.

$$
\text { BACK }
$$

\section{CLOSE WINDOW}

(c) 2021 Copyright - All Rights Reserved | Copyright Clearance Center, Inc. | Privacy statement | Terms and Conditions Comments? We would like to hear from you. E-mail us at customercare@copyright.com 


\section{APS American Physical Society physics seuse and Permissions License}

15-Mar-2021

This license agreement between the American Physical Society ("APS") and Saeed Amiri ("You") consists of your license details and the terms and conditions provided by the American Physical Society and SciPris.

\section{Licensed Content Information}

License Number:

License date:

DOI:

Title:

Author:

Publication:

Publisher:

Cost:
RNP/21/MAR/037634

15-Mar-2021

10.1103/PhysRevLett.114.108302

Friction Boosted by Equilibrium Misalignment of Incommensurate Two-Dimensional Colloid Monolayers

Davide Mandelli et al.

Physical Review Letters

American Physical Society

USD \$ 0.00

\section{$\underline{\text { Request Details }}$}

Does your reuse require significant modifications: No

Specify intended distribution Worldwide

locations:

Reuse Category:

Reuse in a thesis/dissertation

Requestor Type:

Student

Items for Reuse:

Figures/Tables

Number of Figure/Tables:

2

Figure/Tables Details:

FIG. 3 (color online). Equilibrium configurations obtained FIG. 4 (color online). Moiré patterns of the particle monolayer's central region as obtained in $\mathrm{OBC}$

Format for Reuse:

Electronic and Print

Total number of print copies:

Up to 1000

Information about New Publication:

University/Publisher:

Title of dissertation/thesis:

Author(s):

Expected completion date:

\section{License Requestor Information}

Name:

Saeed Amiri

Affiliation:

Email Id:

Country: freedom

Saeed Amiri

Jun. 2021

Individual

Germany

\section{Georg-August-Universität Göttingen}

Friction under active f control in systems with tailored degrees of

sdamiri7@gmail.com 74 


\title{
APS American Physical Society physics Reuse and Permissions License
}

\author{
28-Mar-2021
}

This license agreement between the American Physical Society ("APS") and Saeed Amiri ("You") consists of your license details and the terms and conditions provided by the American Physical Society and SciPris.

\section{Licensed Content Information}

License Number:

License date:

DOI:

Title:

Author:

Publication:

Publisher:

Cost:
RNP/21/MAR/038163

28-Mar-2021

10.1103/PhysRevLett.102.136102

Temperature-Induced Enhancement of Nanoscale Friction

Z. Tshiprut, S. Zelner, and M. Urbakh

Physical Review Letters

American Physical Society

USD $\$ 0.00$

\section{$\underline{\text { Request Details }}$}

Does your reuse require significant modifications: No

Specify intended distribution Worldwide locations:

Reuse in a thesis/dissertation

Reuse Category:

Student

Requestor Type:

Figures/Tables

Items for Reuse:

2

Number of Figure/Tables:

FIG. 1 Temperature dependences FIG. 3 Temperature dependences

Figure/Tables Details:

Electronic and Print

Format for Reuse:

Up to 1000

Total number of print copies:

Information about New Publication:

University/Publisher:

Title of dissertation/thesis:

Author(s):

Expected completion date:

License Requestor Information

Name:

Affiliation:

Email Id:

Country:
Institut für a Materialphysik, Göttingen

Friction under active control in systems with tailored degrees of freedom

Saeed Amiri

Sep. 2021

Saeed Amiri

Individual

sdamiri7@gmail.com

Germany 


\begin{tabular}{|c|c|}
\hline \multirow{5}{*}{ SPRINGER NATURE } & Ballistic nanofriction \\
\hline & Author: Roberto Guerra et al \\
\hline & Publication: Nature Materials \\
\hline & Publisher: Springer Nature \\
\hline & Date: Jul 18, 2010 \\
\hline
\end{tabular}

\section{Order Completed}

Thank you for your order.

This Agreement between Mr. Saeed Amiri ("You") and Springer Nature ("Springer Nature") consists of your license details and the terms and conditions provided by Springer Nature and Copyright Clearance Center.

Your confirmation email will contain your order number for future reference.

License Number $5035790055082 \quad$ 白 Printable Details

License date Mar 25, 2021

\section{Licensed Content}

\section{Licensed Content}

Publisher

Licensed Content

Publication

Licensed Content

Title

Licensed Content

Author

Licensed Content

Date

\section{Springer Nature}

Nature Materials

Ballistic nanofriction

Roberto Guerra et al

Jul 18, 2010
About Your Work

\section{Title}

Expected

presentation date

in systems with tailored

degrees of freedom

Institut für a Materialphysik Göttingen

Jun 2021

\section{Requestor Location}

Mr. Saeed Amir

Ginsterweg

Requestor Location

\section{Göttingen, 37077}

Germany

Attn: Mr. Saeed Amiri

\section{\$ Price}

Total

0.00 USD

\section{首 Order Details}

$\begin{array}{ll}\begin{array}{l}\text { Type of Use } \\ \text { Requestor type }\end{array} & \begin{array}{l}\text { Thesis/Dissertation } \\ \text { non-commercial (non-profit) } \\ \text { Format }\end{array} \\ \begin{array}{l}\text { print and electronic } \\ \text { figures/tables/illustrations }\end{array} \\ \begin{array}{l}\text { Number of } \\ \text { figures/tables/illustrations } \\ \text { High-res required }\end{array} \\ \begin{array}{l}\text { Will you be } \\ \text { translating? }\end{array} \\ \begin{array}{l}\text { Circulation/distribution } \\ \text { Author of this } \\ \text { Springer Nature } \\ \text { content }\end{array} \\ \begin{array}{l}\text { F } \\ \text { Additional Data }\end{array}\end{array}$

Figure 2 | Thermal diffusion of deposited Au clusters on graphite Figure 4 | Slowdown dynamics of kicked $\mathrm{Au}$ clusters Figure 5 | Time decay of kinetic energies of purely sliding and of sliding/rotating ballistic Au clusters

䀚 Tax Details

Total: 0.00 USD 


\begin{tabular}{|l|l} 
& Velocity tuning of friction with two trapped atoms \\
& Author: Dorian Gangloff et al \\
SPRINGER NATURE & Publication: Nature Physics \\
& Publisher: Springer Nature \\
& Date: Sep 14, 2015 \\
& Copyright 0 2015, Nature Publishing Group
\end{tabular}

\section{Order Completed}

\section{Thank you for your order.}

This Agreement between Mr. Saeed Amiri ("You") and Springer Nature ("Springer Nature") consists of your license details and the terms and conditions provided by Springer Nature and Copyright Clearance Center.

Your confirmation email will contain your order number for future reference.

License Number $5034130020672 \quad$ 王 Printable Details

License date Mar 22, 2021

\section{$\checkmark$ Licensed Content}

$\begin{array}{ll}\begin{array}{l}\text { Licensed Content } \\ \text { Publisher } \\ \text { Licensed Content } \\ \text { Publication }\end{array} & \text { Springer Nature } \\ \begin{array}{l}\text { Licensed Content } \\ \text { Title }\end{array} & \text { Nature Physics } \\ \text { Licensed Content } & \begin{array}{l}\text { Velocity tuning of friction } \\ \text { with two trapped atoms }\end{array} \\ \begin{array}{l}\text { Licensed Content } \\ \text { Date }\end{array} & \text { Dorian Gangloff et al } \\ \end{array}$

Date

About Your Work

Title

Expected

presentation date

Requestor Location

Friction under active $\mathrm{f}$ control

in systems with tailored

degrees of freedom

Institut für a Materialphysik, Göttingen

Jun 2021

Mr. Saeed Amir

Ginsterweg

Requestor Location

Göttingen, 37077

Germany

Attn: Mr. Saeed Amir

\$ Price

$\begin{array}{ll}\text { Total } & 0.00 \text { EUR }\end{array}$

\section{首 Order Details}

\begin{tabular}{|c|c|}
\hline Type of Use & Thesis/Dissertation \\
\hline Requestor type & non-commercial (non-profit) \\
\hline Format & print and electronic \\
\hline Portion & figures/tables/illustrations \\
\hline $\begin{array}{l}\text { Number of } \\
\text { figures/tables/illustrations }\end{array}$ & 2 \\
\hline High-res required & no \\
\hline $\begin{array}{l}\text { Will you be } \\
\text { translating? }\end{array}$ & no \\
\hline Circulation/distribution & $100-199$ \\
\hline $\begin{array}{l}\text { Author of this } \\
\text { Springer Nature } \\
\text { content }\end{array}$ & no \\
\hline \multicolumn{2}{|l|}{$F$ Additional Data } \\
\hline Portions & $\begin{array}{l}\text { Figure } 2 \text { | Velocity } \\
\text { dependence of stick-slip } \\
\text { friction for one atom Figure } 4 \\
\text { | Structural and thermal } \\
\text { lubricity of two atoms. }\end{array}$ \\
\hline 具 Tax Details & \\
\hline
\end{tabular}

Total: 0.00 EUR 


\section{APS American Physical Society physics Reuse and Permissions License}

$11-$ Oct-2021

This license agreement between the American Physical Society ("APS") and Saeed Amiri ("You") consists of your license details and the terms and conditions provided by the American Physical Society and SciPris.

\section{Licensed Content Information}

License Number:

License date:

DO I:

Title:

Author:

Publication:

Publisher:

Cost:
RNP/21/O C T/045403

11-Oct-2021

10.1103/PhysRevE.104.014802

Friction on incommensurate substrates: Role of anharmonicity and defects

S. Amiri, C. A. Volkert, and R. L. C. Vink

Physical Review E

American Physical Society

USD \$ 0.00

\section{$\underline{\text { Request Details }}$}

Does your reuse require significant modifications: Yes

Modification Details:

Specify intended distribution locations:

Reuse Category:

Requestor Type:

Items for Reuse:

Format for Reuse:

Total number of print copies:
Worldwide

Reuse in a thesis/dissertation

Author of requested content

Whole Article

Print and Electronic

Up to 1000

Information about New Publication:

University/Publisher:

Title of dissertation/thesis:

Author(s):

Expected completion date:
Georg-August-Universität

Friction under active control in systems with tailored degrees of freedom

Saeed Amiri

Nov. 2021

\section{License Requestor Information}

Name:

Affiliation:

Email Id:

Country:
Saeed Amiri

Individual

sdamiri7@gmail.com

Germany 


\section{Bibliography}

[1] K. Shinjo and M. Hirano. Dynamics of friction: superlubric state. Surface Science, 283(1-3):473-478, Mar 1993.

[2] M. Urbakh, J. Klafter, D. Gourdon, and J. Israelachvili. The nonlinear nature of friction. Nature, 430(6999):525-528, Jul 2004.

[3] B.N.J. Persson. Theory of friction:mfriction dynamics for boundary lubricated surfaces. Physical Review B, 55(12):8004-8004, Mar 1997.

[4] B. Persson. Sliding friction. Surface Science Reports, 33(3):83-119, 1999.

[5] H. Jost. Tribology — origin and future. Wear, 136(1):1-17, Feb 1990.

[6] P.J. Blau. Friction Science and Technology: From concepts to applications, second edition. CRC Press, 2008-10-20. ISBN:9781420054101.

[7] A. Fall, B. Weber, M. Pakpour, N. Lenoir, N. Shahidzadeh, J. Fiscina, C. Wagner, and D. Bonn. Sliding friction on wet and dry sand. Physical Review Letters, 112(17), Apr 2014.

[8] J.S McFarlane and D. Tabor. Relation between friction and adhesion. Proceedings of the Royal Society of London. Series A. Mathematical and Physical Sciences, 202(1069):244-253, 1950.

[9] L. Zhang and H. Tanaka. Towards a deeper understanding of wear and friction on the atomic scale - a molecular dynamics analysis. Wear, 211(1):44-53, Oct 1997.

[10] K.C. Ludema. Friction, Wear, Lubrication: A textbook in tribology. CRC Press, 1996-06-21. ISBN:9781439821893.

[11] B. Persson and E. Tosatti. Physics of Sliding Friction. Springer, 199602-29. ISBN:9780792339359. 
[12] C.K. Curtis, J.L. Streator, and J. Krim. Friction, Jan 2020. Wiley.

[13] J. Klafter and M. Urbakh. The basic of nanoscale friction and ways to control it. In Fundamentals of Friction and Wear, pages 143-158. Springer Berlin Heidelberg, 2007.

[14] F. Elmer. Controlling friction. Physical Review E, 57(5):R4903-R4906, May 1998.

[15] K. Holmberg and A. Erdemir. Influence of tribology on global energy consumption, costs and emissions. Friction, 5(3):263-284, Sep 2017.

[16] R.W. Carpick. Controlling friction. Science, 313(5784):184-185, Jul 2006.

[17] Y. Braiman, J. Barhen, and V. Protopopescu. Control of friction at the nanoscale. Physical Review Letters, 90(9), Mar 2003.

[18] J.N. Israelachvili. Friction and lubrication forces. In Intermolecular and Surface Forces, pages 469-499. Elsevier, 2011.

[19] M. Otsuki and H. Matsukawa. Systematic breakdown of amontons' law of friction for an elastic object locally obeying amontons' law. Scientific Reports, 3(1), Apr 2013.

[20] B. Luan and M.O. Robbins. The breakdown of continuum models for mechanical contacts. Nature, 435(7044):929-932, Jun 2005.

[21] J. Gao, W.D. Luedtke, D. Gourdon, M. Ruths, J.N. Israelachvili, and U. Landman. Frictional forces and amontons' law: from the molecular to the macroscopic scale. The Journal of Physical Chemistry B, 108(11):3410-3425, Feb 2004.

[22] Y. Mo, K.T. Turner, and I. Szlufarska. Friction laws at the nanoscale. Nature, 457(7233):1116-1119, Feb 2009.

[23] M.H. Müser. Structural lubricity: role of dimension and symmetry. Europhysics Letters (EPL), 66(1):97-103, Apr 2004.

[24] M. Cieplak, E.D. Smith, and M.O. Robbins. Molecular origins of friction: the force on adsorbed layers. Science, 265(5176):1209-1212, Aug 1994 .

[25] A. Vanossi, N. Manini, M. Urbakh, S. Zapperi, and E. Tosatti. Colloquium: modeling friction: from nanoscale to mesoscale. Reviews of Modern Physics, 85(2):529-552, Apr 2013. 
[26] C. Fusc, R. Smith, M. Urbakh, and A. Vanossi. Friction at the nanoscale. Journal of Physics: Condensed Matter, 20(35):350301, Aug 2008.

[27] I. Szlufarska, M. Chandross, and R.W. Carpick. Recent advances in single-asperity nanotribology. Journal of Physics D: Applied Physics, 41(12):123001, May 2008.

[28] J. Krim. Friction and energy dissipation mechanisms in adsorbed molecules and molecularly thin films. Advances in Physics, 61(3):155323, Jun 2012.

[29] S.Y. Krylov, K.B. Jinesh, H. Valk, M. Dienwiebel, and J.W.M. Frenken. Thermally induced suppression of friction at the atomic scale. Physical Review E, 71(6), Jun 2005.

[30] E. Gnecco, R. Bennewitz, T. Gyalog, and E. Meyer. Friction experiments on the nanometre scale. Journal of Physics: Condensed Matter, 13(31):R619-R642, Jul 2001.

[31] V.L. Popov and J.A.T. Gray. Prandtl-tomlinson model: a simple model which made history. In The History of Theoretical, Material and Computational Mechanics - Mathematics Meets Mechanics and Engineering, pages 153-168. Springer Berlin Heidelberg, 2014.

[32] M.H. Müser, M. Urbakh, and M.O. Robbins. Statistical mechanics of static and Low-Velocity kinetic friction. In Advances in Chemical Physics, pages 187-272. John Wiley \& Sons, Inc., Mar 2003.

[33] L. Prandtl. Ein gedankenmodell zur kinetischen theorie der festen körper. ZAMM - Zeitschrift für Angewandte Mathematik und Mechanik, 8(2):85-106, 1928. [A conceptual model to the kinetic theory of solid bodies. by L. Prandtl in Göttingen. (Translated from German original by V. L. Popov and J. Gray, Berlin University of Technology, 2012)]

[34] O.M. Braun and Y. Kivshar. The Frenkel-Kontorova Model: Concepts, methods, and applications. Springer, 2010-10-19. ISBN:9783642073977.

[35] R. Pynn. Incommensurable structures. Nature, 281(5731):433-437, Oct 1979.

[36] P. Rosenau. Dynamics of nonlinear mass-spring chains near the continuum limit. Physics Letters A, 118(5):222-227, Oct 1986. 
[37] A. Vanossi, J. Röder, A.R. Bishop, and V. Bortolani. Underdamped commensurate dynamics in a driven Frenkel-Kontorova-type model. Physical Review E, 67(1), Jan 2003.

[38] A. Vanossi and O.M. Braun. Driven dynamics of simplified tribological models. Journal of Physics: Condensed Matter, 19(30):305017, Jul 2007 .

[39] M. Weiss and F. Elmer. Dry friction in the Frenkel-KontorovaTomlinson model: static properties. Physical Review B, 53(11):75397549, Mar 1996.

[40] M. Weiss and F. Elmer. Dry friction in the Frenkel-KontorovaTomlinson model: dynamical properties. Zeitschrift für Physik B Condensed Matter, 104(1):55-69, Jan 1997.

[41] A. Milchev and G.M. Mazzucchelli. Frenkel-kontorova model with anharmonic interactions. Physical Review B, 38(4):2808-2812, Aug 1988.

[42] O.M. Braun and Y.S. Kivshar. Nonlinear dynamics of the FrenkelKontorova model. Physics Reports, 306(1-2):1-108, Dec 1998.

[43] O.M. Braun, H. Zhang, B. Hu, and J. Tekic. Driven kinks in the anharmonic Frenkel-Kontorova model. Physical Review E, 67(6), Jun 2003.

[44] O.M. Braun, Y.S. Kivshar, and I.I. Zelenskaya. Kinks in the FrenkelKontorova model with long-range interparticle interactions. Physical Review B, 41(10):7118-7138, Apr 1990.

[45] O.M. Braun. Supersonic and multiple topological excitations in the driven Frenkel-Kontorova model with exponential interaction. Physical Review E, 62(5):7315-7319, Nov 2000.

[46] P.S. Lomdahl and D.J. Srolovitz. Dislocation generation in the TwoDimensional Frenkel-Kontorova model at high stresses. Physical Review Letters, 57(21):2702-2705, Nov 1986.

[47] J. Röder, J.E. Hammerberg, B.L. Holian, and A.R. Bishop. Multichain Frenkel-Kontorova model for interfacial slip. Physical Review B, 57(5):2759-2766, Feb 1998.

[48] J. Hammerberg, B. Holian, J. Röder, A. Bishop, and S. Zhou. Nonlinear dynamics and the problem of slip at material interfaces. Physica D: Nonlinear Phenomena, 123(1-4):330-340, Nov 1998. 
[49] O.M. Braun and Y.S. Kivshar. Zigzag kinks in the Frenkel-Kontorova model with a transversal degree of freedom. Physical Review B, 44(14):7694-7697, Oct 1991.

[50] P.L. Christiansen, A.V. Savin, and A.V. Zolotaryuk. Zig-zag version of the Frenkel-Kontorova model. Physical Review B, 54(18):12892-12902, Nov 1996.

[51] O.M. Braun, M.V. Paliy, J. Röder, and A.R. Bishop. Lockedto-running transition in the two-dimensional underdamped driven Frenkel-Kontorova model. Physical Review E, 63(3), Feb 2001.

[52] Y.N. Gornostyrev, M.I. Katsnelson, A.V. Kravtsov, and A.V. Trefilov. Kink nucleation in the two-dimensional Frenkel-Kontorova model. Physical Review E, 66(2), Aug 2002.

[53] O.M. Braun, B. Hu, A. Filippov, and A. Zeltser. Traffic jams and hysteresis in driven one-dimensional systems. Physical Review E, 58(2):1311-1324, Aug 1998.

[54] O.M. Braun, I. Barel, and M. Urbakh. Dynamics of transition from static to kinetic friction. Physical Review Letters, 103(19), Nov 2009.

[55] H. Ghorbanfekr-Kalashami, F.M. Peeters, K.S. Novoselov, and M. Neek-Amal. Spatial design and control of graphene flake motion. Physical Review B, 96(6), Aug 2017.

[56] F. Bonelli, N. Manini, E. Cadelano, and L. Colombo. Atomistic simulations of the sliding friction of graphene flakes. The European Physical Journal B, 70(4):449-459, Jul 2009.

[57] Y. Dong, Z. Duan, Y. Tao, Z. Wei, B. Gueye, Y. Zhang, and Y. Chen. Friction evolution with transition from commensurate to incommensurate contacts between graphene layers. Tribology International, 136:259-266, Aug 2019.

[58] G.S. Verhoeven, M. Dienwiebel, and J.W.M. Frenken. Model calculations of superlubricity of graphite. Physical Review B, 70(16), Oct 2004 .

[59] A.D. Novaco and J.P. McTague. Orientational epitaxy - the orientational ordering of incommensurate structures. Physical Review Letters, 38(22):1286-1289, May 1977. 
[60] I.V. Lebedeva and A.M. Popov. Energetics and structure of domain wall networks in minimally twisted bilayer graphene under strain. The Journal of Physical Chemistry C, 124(3):2120-2130, Dec 2019.

[61] I.V. Lebedeva and A.M. Popov. Commensurate-incommensurate phase transition and a network of domain walls in bilayer graphene with a biaxially stretched layer. Physical Review B, 99(19), May 2019.

[62] T. Gyalog and H. Thomas. Friction between atomically flat surfaces. Europhysics Letters (EPL), 37(3):195-200, Jan 1997.

[63] C.R. Woods, L. Britnell, A. Eckmann, R.S. Ma, J.C. Lu, H.M. Guo, X. Lin, G.L. Yu, Y. Cao, R. Gorbachev, A.V. Kretinin, J. Park, L.A. Ponomarenko, M.I. Katsnelson, Y. Gornostyrev, K. Watanabe, T. Taniguchi, C. Casiraghi, H. Gao, A.K. Geim, and K. Novoselov. Commensurate-incommensurate transition in graphene on hexagonal boron nitride. Nature Physics, 10(6):451-456, Apr 2014.

[64] M. Peyrard and S. Aubry. Critical behaviour at the transition by breaking of analyticity in the discrete Frenkel-Kontorova model. Journal of Physics C: Solid State Physics, 16(9):1593-1608, Mar 1983.

[65] S. Aubry and P.L. Daeron. The discrete Frenkel-Kontorova model and its extensions. Physica D: Nonlinear Phenomena, 8(3):381-422, Sep 1983.

[66] Y. Braiman, J. Baumgarten, J. Jortner, and J. Klafter. Symmetrybreaking transition in finite Frenkel-Kontorova chains. Physical Review Letters, 65(19):2398-2401, Nov 1990.

[67] M. Hirano and K. Shinjo. Atomistic locking and friction. Physical Review B, 41(17):11837-11851, Jun 1990.

[68] M. Dienwiebel and J.W.M. Frenken. Experimental observations of superlubricity and thermolubricity. In Fundamentals of Friction and Wear, pages 159-176. Springer Berlin Heidelberg, 2007.

[69] M.H. Müser. Theoretical aspects of superlubricity. In Fundamentals of Friction and Wear, pages 177-199. Springer Berlin Heidelberg, 2007.

[70] A.E. Filippov, M. Dienwiebel, J.W.M. Frenken, J. Klafter, and M. Urbakh. Torque and twist against superlubricity. Physical Review Letters, 100(4), Jan 2008. 
[71] M. Hirano, K. Shinjo, R. Kaneko, and Y. Murata. Anisotropy of frictional forces in muscovite mica. Physical Review Letters, 67(19):26422645, Nov 1991.

[72] J.B. Sokoloff. Possible nearly frictionless sliding for mesoscopic solids. Physical Review Letters, 71(21):3450-3453, Nov 1993.

[73] J.B. Sokoloff. Microscopic mechanisms for kinetic friction: nearly frictionless sliding for small solids. Physical Review B, 52(10):7205-7214, Sep 1995.

[74] F. Grey and J. Bohr. A symmetry principle for epitaxial rotation. Europhysics Letters (EPL), 18(8):717-722, Apr 1992.

[75] J.M. Martin, C. Donnet, T.L. Mogne, and T. Epicier. Superlubricity of molybdenum disulphide. Physical Review B, 48(14):10583-10586, Oct 1993.

[76] I. Amidror. Introduction. In Computational Imaging and Vision, pages 1-8. Springer Netherlands, 2000.

[77] D.W. Pashley, J.W. Menter, and G.A. Bassett. Observation of dislocations in metals by means of moiré patterns on electron micrographs. Nature, 179(4563):752-755, Apr 1957.

[78] H.S. Wong, C. Durkan, and N. Chandrasekhar. Tailoring the local interaction between graphene layers in graphite at the atomic scale and above using scanning tunneling microscopy. ACS Nano, 3(11):34553462 , Oct 2009.

[79] M. Mirzakhani, F.M. Peeters, and M. Zarenia. Circular quantum dots in twisted bilayer graphene. Physical Review B, 101(7), Feb 2020.

[80] O. Kafri and I. Glatt. The Physics of Moire Metrology. WileyInterscience, 1990. ISBN:0471509671.

[81] W. Li, C.J.O. Reichhardt, B. Jankó, and C. Reichhardt. Vortex dynamics, pinning, and magic angles on moiré patterns. arXiv, Dec. 2021. cond-mat.supr-con.

[82] Y. Song, D. Mandelli, O. Hod, M. Urbakh, M. Ma, and Q. Zheng. Robust microscale superlubricity in graphite/hexagonal boron nitride layered heterojunctions. Nature Materials, 17(10):894-899, Jul 2018. 
[83] S. Zhang, T. Ma, A. Erdemir, and Q. Li. Tribology of two-dimensional materials: from mechanisms to modulating strategies. Materials Today, 26:67-86, Jun 2019.

[84] E. Koren and U. Duerig. Moiré scaling of the sliding force in twisted bilayer graphene. Physical Review B, 94(4), Jul 2016.

[85] M. Dienwiebel, G.S. Verhoeven, N. Pradeep, J.W.M. Frenken, J.A. Heimberg, and H.W. Zandbergen. Superlubricity of graphite. Physical Review Letters, 92(12), Mar 2004.

[86] D. Mandelli, I. Leven, O. Hod, and M. Urbakh. Sliding friction of graphene/hexagonal -boron nitride heterojunctions: a route to robust superlubricity. Scientific Reports, 7(1), Sep 2017.

[87] X. Feng, S. Kwon, J.Y. Park, and M. Salmeron. Superlubric sliding of graphene nanoflakes on graphene. ACS Nano, 7(2):1718-1724, Jan 2013 .

[88] R.C. Sinclair, J.L. Suter, and P.V. Coveney. Graphene-graphene interactions: friction, superlubricity, and exfoliation. Advanced Materials, 30(13):1705791, Feb 2018.

[89] A.S. de Wijn, C. Fusco, and A. Fasolino. Stability of superlubric sliding on graphite. Physical Review E, 81(4), Apr 2010.

[90] A.S. de Wijn, A. Fasolino, A.E. Filippov, and M. Urbakh. Low friction and rotational dynamics of crystalline flakes in solid lubrication. EPL (Europhysics Letters), 95(6):66002, Aug 2011.

[91] I. Leven, D. Krepel, O. Shemesh, and O. Hod. Robust superlubricity in Graphene/h-BN heterojunctions. The Journal of Physical Chemistry Letters, 4(1):115-120, Dec 2012.

[92] A. Vanossi, C. Bechinger, and M. Urbakh. Structural lubricity in soft and hard matter systems. Nature Communications, 11(1), Sep 2020.

[93] D. Dietzel, J. Brndiar, I. Štich, and A. Schirmeisen. Limitations of structural superlubricity: chemical bonds versus contact size. ACS Nano, 11(8):7642-7647, Jul 2017.

[94] O. Hod, E. Meyer, Q. Zheng, and M. Urbakh. Structural superlubricity and ultralow friction across the length scales. Nature, 563(7732):485492, Nov 2018. 
[95] C. Qu, K. Wang, J. Wang, Y. Gongyang, R.W. Carpick, M. Urbakh, and Q. Zheng. Origin of friction in superlubric graphite contacts. Physical Review Letters, 125(12), Sep 2020.

[96] M.Z. Baykara, M.R. Vazirisereshk, and A. Martini. Emerging superlubricity: a review of the state of the art and perspectives on future research. Applied Physics Reviews, 5(4):041102, Dec 2018.

[97] T. Bohlein, J. Mikhael, and C. Bechinger. Observation of kinks and antikinks in colloidal monolayers driven across ordered surfaces. Nature Materials, 11(2):126-130, Dec 2011.

[98] A. Vanossi and E. Tosatti. Kinks in motion. Nature Materials, 11(2):97-98, Jan 2012.

[99] A. Vanossi, N. Manini, and E. Tosatti. Static and dynamic friction in sliding colloidal monolayers. Proceedings of the National Academy of Sciences, 109(41):16429-16433, Sep 2012.

[100] D. Mandelli, A. Vanossi, N. Manini, and E. Tosatti. Friction boosted by equilibrium misalignment of incommensurate Two-Dimensional colloid monolayers. Physical Review Letters, 114(10), Mar 2015.

[101] S. Bleil, H.H. von Grünberg, J. Dobnikar, R. Castañeda-Priego, and C. Bechinger. Strain-induced domain formation in two-dimensional colloidal systems. Europhysics Letters (EPL), 73(3):450-456, Feb 2006.

[102] D. Mandelli, A. Vanossi, M. Invernizzi, S. Paronuzzi, N. Manini, and E. Tosatti. Superlubric-pinned transition in sliding incommensurate colloidal monolayers. Physical Review B, 92(13), Oct 2015.

[103] D. Mandelli, A. Vanossi, N. Manini, and E. Tosatti. Finite-temperature phase diagram and critical point of the aubry pinned-sliding transition in a two-dimensional monolayer. Physical Review B, 95(24), Jun 2017.

[104] M. Cetina, A. Bylinskii, L. Karpa, D. Gangloff, K.M. Beck, Y. Ge, M. Scholz, A.T. Grier, I. Chuang, and V. Vuletić. One-dimensional array of ion chains coupled to an optical cavity. New Journal of Physics, 15(5):053001, May 2013.

[105] D. Gangloff, A. Bylinskii, I. Counts, W. Jhe, and V. Vuletić. Velocity tuning of friction with two trapped atoms. Nature Physics, 11(11):915919, Sep 2015. 
[106] E. Meyer. Controlling friction atom by atom. Science, 348(6239):10891089, Jun 2015.

[107] D. Mandelli and E. Tosatti. Microscopic friction emulators. Nature, 526(7573):332-333, Oct 2015.

[108] A. Bylinskii, D. Gangloff, I. Counts, and V. Vuletić. Observation of Aubry-type transition in finite atom chains via friction. Nature Materials, 15(7):717-721, Mar 2016.

[109] I. Counts, D. Gangloff, A. Bylinskii, J. Hur, R. Islam, and V. Vuletić. Multislip friction with a single ion. Physical Review Letters, 119(4), Jul 2017.

[110] X. Cao, E. Panizon, A. Vanossi, N. Manini, and C. Bechinger. Orientational and directional locking of colloidal clusters driven across periodic surfaces. Nature Physics, 15(8):776-780, May 2019.

[111] P. Tierno. A moiré foray. Nature Physics, 15(8):733-733, May 2019.

[112] X. Cao, E. Panizon, A. Vanossi, N. Manini, E. Tosatti, and C. Bechinger. Pervasive orientational and directional locking at geometrically heterogeneous sliding interfaces. Physical Review E, 103(1), Jan 2021.

[113] R.L. Stoop, A.V. Straube, T.H. Johansen, and P. Tierno. Collective directional locking of colloidal monolayers on a periodic substrate. Physical Review Letters, 124(5), Feb 2020.

[114] S.G. Leyva, R.L. Stoop, P. Tierno, and I. Pagonabarraga. Dynamics and clogging of colloidal monolayers magnetically driven through a heterogeneous landscape. Soft Matter, 16(30):6985-6992, 2020.

[115] T. Brazda, A. Silva, N. Manini, A. Vanossi, R. Guerra, E. Tosatti, and C. Bechinger. Experimental observation of the aubry transition in Two-Dimensional colloidal monolayers. Physical Review X, 8(1), Mar 2018.

[116] Y. Maruyama. Temperature dependence of Lévy-type stick-slip diffusion of a gold nanocluster on graphite. Physical Review B, 69(24), Jun 2004 .

[117] X. Zhao, S.R. Phillpot, W.G. Sawyer, S.B. Sinnott, and S.S. Perry. Transition from thermal to athermal friction under cryogenic conditions. Physical Review Letters, 102(18), May 2009. 
[118] A. Schirmeisen, L. Jansen, H. Hölscher, and H. Fuchs. Temperature dependence of point contact friction on silicon. Applied Physics Letters, 88(12):123108, Mar 2006.

[119] Z. Tshiprut, S. Zelner, and M. Urbakh. Temperature-induced enhancement of nanoscale friction. Physical Review Letters, 102(13), Apr 2009.

[120] I. Barel, M. Urbakh, L. Jansen, and A. Schirmeisen. Multibond dynamics of nanoscale friction: the role of temperature. Physical Review Letters, 104(6), Feb 2010.

[121] A.E. Filippov, J. Klafter, and M. Urbakh. Friction through dynamical formation and rupture of molecular bonds. Physical Review Letters, 92(13), Mar 2004.

[122] Y. Dong, D. Perez, H. Gao, and A. Martini. Thermal activation in atomic friction: revisiting the theoretical analysis. Journal of Physics: Condensed Matter, 24(26):265001, May 2012.

[123] J. Hasnain, S. Jungblut, A. Tröster, and C. Dellago. Frictional dynamics of stiff monolayers: from nucleation dynamics to thermal sliding. Nanoscale, 6(17):10161-10168, 2014.

[124] M. Dienwiebel and J.W.M. Frenken. Experimental observations of superlubricity and thermolubricity. In Fundamentals of Friction and Wear on the Nanoscale, pages 139-156. Springer International Publishing, Nov 2014.

[125] A. Franchini, V. Bortolani, G. Santoro, and M. Brigazzi. Thermal effects in static friction: thermolubricity. Physical Review E, 78(4), Oct 2008 .

[126] K.B. Jinesh, S.Y. Krylov, H. Valk, M. Dienwiebel, and J.W.M. Frenken. Thermolubricity in atomic-scale friction. Physical Review B, 78(15), Oct 2008 .

[127] J.Y. Park and M. Salmeron. Fundamental aspects of energy dissipation in friction. Chemical Reviews, 114(1):677-711, Sep 2013.

[128] A. Socoliuc, E. Gnecco, S. Maier, O. Pfeiffer, A. Baratoff, R. Bennewitz, and E. Meyer. Atomic-scale control of friction by actuation of nanometer-sized contacts. Science, 313(5784):207-210, 2006. 
[129] P. Steiner, R. Roth, E. Gnecco, A. Baratoff, S. Maier, T. Glatzel, and E. Meyer. Two-dimensional simulation of superlubricity on nacl and highly oriented pyrolytic graphite. Physical Review B, 79(4), Jan 2009.

[130] E. Riedo, E. Gnecco, R. Bennewitz, E. Meyer, and H. Brune. Interaction potential and hopping dynamics governing sliding friction. Physical Review Letters, 91(8), Aug 2003.

[131] Y. Sang, M. Dubé, and M. Grant. Thermal effects on atomic friction. Physical Review Letters, 87(17), Oct 2001.

[132] S.Y. Krylov and J.W.M. Frenken. The crucial role of temperature in atomic scale friction. Journal of Physics: Condensed Matter, 20(35):354003, Aug 2008.

[133] L. Jansen, H. Hölscher, H. Fuchs, and A. Schirmeisen. Temperature dependence of Atomic-Scale Stick-Slip friction. Physical Review Letters, 104(25), Jun 2010.

[134] Q. Li, Y. Dong, D. Perez, A. Martini, and R.W. Carpick. Speed dependence of atomic Stick-Slip friction in optimally matched experiments and molecular dynamics simulations. Physical Review Letters, 106(12), Mar 2011.

[135] E. Gnecco, R. Bennewitz, T. Gyalog, C. Loppacher, M. Bammerlin, E. Meyer, and H. Güntherodt. Velocity dependence of atomic friction. Physical Review Letters, 84(6):1172-1175, Feb 2000.

[136] T. Bouhacina, J.P. Aimé, S. Gauthier, D. Michel, and V. Heroguez. Tribological behavior of a polymer grafted on silanized silica probed with a nanotip. Physical Review B, 56(12):7694-7703, Sep 1997.

[137] J.D. McGraw, A. Niguès, A. Chennevière, and A. Siria. Contact dependence and velocity crossover in friction between microscopic solid/solid contacts. Nano Letters, 17(10):6335-6339, Sep 2017.

[138] G. He and M.O. Robbins. Simulations of the kinetic friction due to adsorbed surface layers. Tribology Letters, 10(1):7-14, Jan 2001.

[139] C. Fusco and A. Fasolino. Velocity dependence of atomic-scale friction: a comparative study of the one- and two-dimensional tomlinson model. Physical Review B, 71(4), Jan 2005.

[140] M.H. Müser. Velocity dependence of kinetic friction in the PrandtlTomlinson model. Physical Review B, 84(12), Sep 2011. 
[141] E. Granato and S.C. Ying. Non-monotonic velocity dependence of atomic friction. Tribology Letters, 39(3):229-233, Mar 2010.

[142] P. Reimann and M. Evstigneev. Nonmonotonic velocity dependence of atomic friction. Physical Review Letters, 93(23), Dec 2004.

[143] N. Tambe and B. Bhushan. Nanoscale friction-induced phase transformation of diamond-like carbon. Scripta Materialia, 52(8):751-755, Apr 2005.

[144] A. Voevodin, A. Phelps, J. Zabinski, and M. Donley. Friction induced phase transformation of pulsed laser deposited diamond-like carbon. Diamond and Related Materials, 5(11):1264-1269, Nov 1996.

[145] N. Tambe and B. Bhushan. Velocity dependence of nanoscale friction, adhesion and wear. In Springer Handbook of Nanotechnology, pages 1011-1050. Springer Berlin Heidelberg, 2007.

[146] S.B. Sinnott, S. Heo, D.W. Brenner, J.A. Harrison, and D.L. Irving. Computer simulations of Nanometer-Scale indentation and friction. In Springer Handbook of Nanotechnology, pages 955-1011. Springer Berlin Heidelberg, 2010.

[147] F. Ptak, C.M. Almeida, and R. Prioli. Velocity-dependent friction enhances tribomechanical differences between monolayer and multilayer graphene. Scientific Reports, 9(1), Oct 2019.

[148] O. Acikgoz and M.Z. Baykara. Speed dependence of friction on singlelayer and bulk mos2 measured by atomic force microscopy. Applied Physics Letters, 116(7):071603, Feb 2020.

[149] W. Ouyang, S.N. Ramakrishna, A. Rossi, M. Urbakh, N.D. Spencer, and A. Arcifa. Load and velocity dependence of friction mediated by dynamics of interfacial contacts. Physical Review Letters, 123(11), Sep 2019.

[150] C. Apostoli, G. Giusti, J. Ciccoianni, G. Riva, R. Capozza, R.L. Woulaché, A. Vanossi, E. Panizon, and N. Manini. Velocity dependence of sliding friction on a crystalline surface. Beilstein Journal of Nanotechnology, 8:2186-2199, Oct 2017.

[151] J.A. van den Ende, A.S. de Wijn, and A. Fasolino. The effect of temperature and velocity on superlubricity. Journal of Physics: Condensed Matter, 24(44):445009, Oct 2012. 
[152] J. Li, T. Wang, N. Liu, and T. Yu. Dependence of the sliding distance of a one-dimensional atom chain on initial velocity. Scientific Reports, 7(1), Nov 2017.

[153] J. Norell, A. Fasolino, and A.S. de Wijn. Emergent friction in twodimensional Frenkel-Kontorova models. Physical Review E, 94(2), Aug 2016 .

[154] M. Evstigneev and P. Reimann. Velocity dependence of atomic friction: rate theory and beyond. In Fundamentals of Friction and Wear, pages 117-142. Springer Berlin Heidelberg, 2007.

[155] R.L.C. Vink. Connection between sliding friction and phonon lifetimes: Thermostat-induced thermolubricity effects in molecular dynamics simulations. Physical Review B, 100(9), Sep 2019.

[156] R.L. Davidchack, R. Handel, and M.V. Tretyakov. Langevin thermostat for rigid body dynamics. The Journal of Chemical Physics, 130(23):234101, Jun 2009 .

[157] A. Benassi, A. Vanossi, G.E. Santoro, and E. Tosatti. Parameter-free dissipation in simulated sliding friction. Physical Review B, 82(8), Aug 2010 .

[158] D. Toton, C.D. Lorenz, N. Rompotis, N. Martsinovich, and L. Kantorovich. Temperature control in molecular dynamic simulations of non-equilibrium processes. Journal of Physics: Condensed Matter, 22(7):074205, Feb 2010.

[159] A. Benassi, A. Vanossi, G.E. Santoro, and E. Tosatti. Optimal energy dissipation in sliding friction simulations. Tribology Letters, 48(1):4149, Mar 2012.

[160] D. Frenkel and B. Smit. Understanding Molecular Simulation: From algorithms to applications. Elsevier, 2001-10-19. ISBN:9780080519982.

[161] M. Reguzzoni, M. Ferrario, S. Zapperi, and M.C. Righi. Onset of frictional slip by domain nucleation in adsorbed monolayers. Proceedings of the National Academy of Sciences, 107(4):1311-1316, Dec 2009.

[162] T. Coffey and J. Krim. Impact of substrate corrugation on the sliding friction levels of adsorbed films. Physical Review Letters, 95(7), Aug 2005. 
[163] C. Daly, J. Zhang, and J.B. Sokoloff. Dry friction due to adsorbed molecules. Physical Review Letters, 90(24), Jun 2003.

[164] M. Pierno, L. Bruschi, G. Mistura, G. Paolicelli, A. di Bona, S. Valeri, R. Guerra, A. Vanossi, and E. Tosatti. Frictional transition from superlubric islands to pinned monolayers. Nature Nanotechnology, 10(8):714-718, May 2015.

[165] R. Guerra, U. Tartaglino, A. Vanossi, and E. Tosatti. Ballistic nanofriction. Nature Materials, 9(8):634-637, Jul 2010.

[166] A. Schirmeisen. Surfing on graphite waves. Nature Materials, 9(8):615616, Aug 2010.

[167] H. Hedgeland, P. Fouquet, A.P. Jardine, G. Alexandrowicz, W. Allison, and J. Ellis. Measurement of single-molecule frictional dissipation in a prototypical nanoscale system. Nature Physics, 5(8):561-564, Jul 2009.

[168] Y. Liu, F. Grey, and Q. Zheng. The high-speed sliding friction of graphene and novel routes to persistent superlubricity. Scientific Reports, 4(1), May 2014.

[169] F. Peymanirad, S.K. Singh, H. Ghorbanfekr-Kalashami, K.S. Novoselov, F.M. Peeters, and M. Neek-Amal. Thermal activated rotation of graphene flake on graphene. 2D Materials, 4(2):025015, Feb 2017.

[170] D. Wang, G. Chen, C. Li, M. Cheng, W. Yang, S. Wu, G. Xie, J. Zhang, J. Zhao, X. Lu, P. Chen, G. Wang, J. Meng, J. Tang, R. Yang, C. He, D. Liu, D. Shi, K. Watanabe, T. Taniguchi, J. Feng, Y. Zhang, and G. Zhang. Thermally induced graphene rotation on hexagonal boron nitride. Physical Review Letters, 116(12), Mar 2016.

[171] I.V. Lebedeva, A.A. Knizhnik, A.M. Popov, O.V. Ershova, Y.E. Lozovik, and B.V. Potapkin. Fast diffusion of a graphene flake on a graphene layer. Physical Review B, 82(15), Oct 2010.

[172] L. Xu, T. Ma, Y. Hu, and H. Wang. Vanishing stick-slip friction in fewlayer graphenes: the thickness effect. Nanotechnology, 22(28):285708, Jun 2011.

[173] P. Avouris and B.N.J. Persson. Excited states at metal surfaces and their non-radiative relaxation. The Journal of Physical Chemistry, 88(5):837-848, Mar 1984. 
[174] W. Dou and J.E. Subotnik. Perspective: how to understand electronic friction. The Journal of Chemical Physics, 148(23):230901, Jun 2018.

[175] Y. Qi, J.Y. Park, B.L.M. Hendriksen, D.F. Ogletree, and M. Salmeron. Electronic contribution to friction on gaas: an atomic force microscope study. Physical Review B, 77(18), May 2008.

[176] I.L. Singer. Friction and energy dissipation at the atomic scale: a review. Journal of Vacuum Science 83 Technology A: Vacuum, Surfaces, and Films, 12(5):2605-2616, Sep 1994.

[177] L. Consoli, H.J.F. Knops, and A. Fasolino. Onset of sliding friction in incommensurate systems. Physical Review Letters, 85(2):302-305, Jul 2000 .

[178] J.N. Glosli and G.M. McClelland. Molecular dynamics study of sliding friction of ordered organic monolayers. Physical Review Letters, 70(13):1960-1963, Mar 1993.

[179] Z. Wang, T. Ma, Y. Hu, L. Xu, and H. Wang. Energy dissipation of atomic-scale friction based on one-dimensional Prandtl-Tomlinson model. Friction, 3(2):170-182, Jun 2015.

[180] J.B. Sokoloff. Excitations of modulated crystals near the commensurate-incommensurate transition. Physical Review B, 25(9):5901-5906, May 1982.

[181] J. Sokoloff. Theory of dynamical friction between idealized sliding surfaces. Surface Science, 144(1):267-272, Aug 1984.

[182] J.B. Sokoloff. Theory of energy dissipation in sliding crystal surfaces. Physical Review B, 42(1):760-765, Jul 1990.

[183] J.B. Sokoloff. Theory of atomic level sliding friction between ideal crystal interfaces. Journal of Applied Physics, 72(4):1262-1270, Aug 1992.

[184] J.B. Sokoloff. Effects of stick-slip motion on energy dissipation in small sliding solids. Journal of Physics: Condensed Matter, 10(44):99919998, Nov 1998.

[185] J.B. Sokoloff. Effects of defects on the friction between film and substrate in a microbalance experiment. Physical Review B, 51(21):1557315574, Jun 1995. 
[186] J.B. Sokoloff. Theory of the effects of multiscale surface roughness and stiffness on static friction. Physical Review E, 73(1), Jan 2006.

[187] J.B. Sokoloff. Surface roughness and dry friction. Physical Review E, 85(2), Feb 2012.

[188] J.B. Sokoloff, J.E. Sacco, and J.F. Weisz. Undamped lattice vibrations in systems with two incommensurate periodicities. Physical Review Letters, 41(22):1561-1564, Nov 1978.

[189] J.E. Sacco, A. Widom, and J.B. Sokoloff. Commensurability in one-dimensional lattices at finite temperature. Journal of Statistical Physics, 21(5):497-516, Nov 1979.

[190] M.S. Tomassone, J.B. Sokoloff, A. Widom, and J. Krim. Dominance of phonon friction for a xenon film on a silver (111) surface. Physical Review Letters, 79(24):4798-4801, Dec 1997.

[191] J.B. Sokoloff, M.S. Tomassone, and A. Widom. Strongly temperature dependent sliding friction for a superconducting interface. Physical Review Letters, 84(3):515-517, Jan 2000.

[192] J. Zhang and J.B. Sokoloff. Adiabatic molecular-dynamics-simulationmethod studies of kinetic friction. Physical Review E, 71(6), Jun 2005.

[193] J.B. Sokoloff and I. Webman. Theory of depinning of monolayer films adsorbed on a quartz crystal microbalance. Physical Review E, 77(4), Apr 2008.

[194] K. Shakouri, J. Behler, J. Meyer, and G. Kroes. Analysis of energy dissipation channels in a benchmark system of activated dissociation: n2 on ru(0001). The Journal of Physical Chemistry C, 122(41):2347023480, Sep 2018.

[195] J. Baumgartl, J. Dietrich, J. Dobnikar, C. Bechinger, and H.H. von Grünberg. Phonon dispersion curves of two-dimensional colloidal crystals: the wavelength-dependence of friction. Soft Matter, 4(11):2199, 2008 .

[196] S.R.S. de Mello, M.E.H.M. da Costa, C.M. Menezes, C.D. Boeira, F.L.F. Jr, F. Alvarez, and C.A. Figueroa. On the phonon dissipation contribution to nanoscale friction by direct contact. Scientific Reports, 7(1), Jun 2017. 
[197] Y. Dong, Y. Tao, R. Feng, Y. Zhang, Z. Duan, and H. Cao. Phonon dissipation in friction with commensurate-incommensurate transition between graphene membranes. Nanotechnology, 31(28):285711, Apr 2020 .

[198] Z. Wei, Z. Duan, Y. Kan, Y. Zhang, and Y. Chen. Phonon energy dissipation in friction between graphene/graphene interface. Journal of Applied Physics, 127(1):015105, Jan 2020.

[199] A. Cammarata and T. Polcar. Control of energy dissipation in sliding low-dimensional materials. Physical Review B, 102(8), Aug 2020.

[200] Z. Xu and P. Huang. Study on the energy dissipation mechanism of atomic-scale friction with composite oscillator model. Wear, 262(78):972-977, Mar 2007.

[201] D. Hull and D.J. Bacon. Introduction to Dislocations. Butterworth Heinemann, 2011. ISBN:9780080966724.

[202] A. Liebsch, S. Gonçalves, and M. Kiwi. Electronic versus phononic friction of xenon on silver. Physical Review B, 60(7):5034-5043, Aug 1999.

[203] M. Igarashi, A. Natori, and J. Nakamura. Size effects in friction of multiatomic sliding contacts. Physical Review B, 78(16), Oct 2008.

[204] K. Ohno, T. Nitta, J. Nakamura, and A. Natori. Atomic scale friction of nanoscale clusters. Journal of Vacuum Science \&5 Technology B: Microelectronics and Nanometer Structures, 22(4):2026, 2004.

[205] Y. Mo, M.H. Müser, and I. Szlufarska. Origin of the isotope effect on solid friction. Physical Review B, 80(15), Oct 2009.

[206] R.J. Cannara, M.J. Brukman, K. Cimatu, A.V. Sumant, S. Baldelli, and R.W. Carpick. Nanoscale friction varied by isotopic shifting of surface vibrational frequencies. Science, 318(5851):780-783, Nov 2007.

[207] A. Lainé, A. Vanossi, A. Niguès, E. Tosatti, and A. Siria. Amplitude nanofriction spectroscopy. Nanoscale, 13(3):1955-1960, 2021.

[208] A. Buldum, D.M. Leitner, and S. Ciraci. Model for phononic energy dissipation in friction. Physical Review B, 59(24):16042-16046, Jun 1999. 
[209] L. Consoli, H.J.F. Knops, and A. Fasolino. Breakdown of a conservation law in incommensurate systems. Physical Review E, 64(1), Jun 2001.

[210] Y. Dong, Q. Li, and A. Martini. Molecular dynamics simulation of atomic friction: a review and guide. Journal of Vacuum Science 85 Technology A: Vacuum, Surfaces, and Films, 31(3):030801, May 2013.

[211] Y. Dong, Q. Li, J. Wu, and A. Martini. Friction, slip and structural inhomogeneity of the buried interface. Modelling and Simulation in Materials Science and Engineering, 19(6):065003, Jul 2011.

[212] B. Njegic and M.S. Gordon. Exploring the effect of anharmonicity of molecular vibrations on thermodynamic properties. The Journal of Chemical Physics, 125(22):224102, Dec 2006.

[213] Harmonic bonds. The true definition of the harmonic bonds to be used in the simulations was implemnted into the LAMMPS package by $\mathrm{R}$. L.C. Vink., 2020.

[214] X. Li and W. E. Variational boundary conditions for molecular dynamics simulations of crystalline solids at finite temperature: treatment of the thermal bath. Physical Review B, 76(10), Sep 2007.

[215] I.V. Lebedeva, A.A. Knizhnik, A.M. Popov, Y.E. Lozovik, and B.V. Potapkin. Interlayer interaction and relative vibrations of bilayer graphene. Physical Chemistry Chemical Physics, 13(13):5687, 2011.

[216] S. Plimpton. Fast parallel algorithms for Short-Range molecular dynamics. Journal of Computational Physics, 117(1):1-19, Mar 1995.

[217] T.S. van Erp, A. Fasolino, O. Radulescu, and T. Janssen. Pinning and phonon localization in Frenkel-Kontorova models on quasiperiodic substrates. Physical Review B, 60(9):6522-6528, Sep 1999.

[218] P.M. Bonetti, A. Rucci, M.L. Chiofalo, and V. Vuletić. Quantum effects in the aubry transition. Physical Review Research, 3(1), Jan 2021.

[219] I. Bahar, T.R. Lezon, A. Bakan, and I.H. Shrivastava. Normal mode analysis of biomolecular structures: functional mechanisms of membrane proteins. Chemical Reviews, 110(3):1463-1497, Sep 2009.

[220] B.N.J. Persson. Comment on "on the origin of frictional energy dissipation". Tribology Letters, 68(1), Jan 2020. 
[221] Y. Dong, A. Vadakkepatt, and A. Martini. Analytical models for atomic friction. Tribology Letters, 44(3):367-386, Sep 2011.

[222] A.S. de Wijn and A. Fasolino. Relating chaos to deterministic diffusion of a molecule adsorbed on a surface. Journal of Physics: Condensed Matter, 21(26):264002, Jun 2009.

[223] A.S. de Wijn. Internal degrees of freedom and transport of benzene on graphite. Physical Review E, 84(1), Jul 2011.

[224] A. W., ilW. Ashcroft, and N.D. Mermin. Solid State Physics. Cengage Learning, 1976. ISBN:0030493463.

[225] M.G. Muraleedharan, K. Gordiz, A. Rohskopf, S.T. Wyant, Z. Cheng, S. Graham, and A. Henry. Understanding phonon transport properties using classical molecular dynamics simulations. arXiv, Dec. 2021. cond-mat.mtrl-sci.

[226] A. McGaughey and M. Kaviany. Phonon transport in molecular dynamics simulations: formulation and thermal conductivity prediction. In Advances in Heat Transfer, pages 169-255. Elsevier, 2006.

[227] A. Stanford and J. Tanner. Oscillations. In Physics for Students of Science and Engineering, pages 210-233. Elsevier, 1985.

[228] T. Strunz and F. Elmer. Driven Frenkel-Kontorova model. i. uniform sliding states and dynamical domains of different particle densities. Physical Review E, 58(2):1601-1611, Aug 1998.

[229] J. Cserti and G. Tichy. A simple model for the vibrational modes in honeycomb lattices. European Journal of Physics, 25(6):723-736, Aug 2004 .

[230] J.O. Bird and P.J. Chivers. Friction. In Newnes Engineering and Physical Science Pocket Book, pages 235-237. Elsevier, 1993.

[231] D. Fekedulegn, M.P. Mac Siúrtáin, M. Pádraig, and J.J Colbert. Parameter estimation of nonlinear models in forestry. Silva Fennica, 33(4):327-336, 1999.

[232] D.D. Joseph and B.H. Yang. Friction factor correlations for laminar, transition and turbulent flow in smooth pipes. Physica D: Nonlinear Phenomena, 239(14):1318-1328, Jul 2010. 
[233] P.G. Gottschalk and J.R. Dunn. The five-parameter logistic: a characterization and comparison with the four-parameter logistic. Analytical Biochemistry, 343(1):54-65, Aug 2005.

[234] L.M. McDowall and R.A.L. Dampney. Calculation of threshold and saturation points of sigmoidal baroreflex function curves. American Journal of Physiology-Heart and Circulatory Physiology, 291(4):H2003H2007, Oct 2006.

[235] H.K. Tam, L.M. Tam, A.J. Ghajar, P.H. Fu, and C. Sun. Development of friction factor correlation for single-phase flow in micro-fin tube using logistic dose response curve fitting method. TC 02-012 (okstate.edu), 2011. Oklahoma State University.

[236] M.J.R. Healy. Statistical analysis of radioimmunoassay data. Biochemical Journal, 130(1):207-210, Nov 1972.

[237] D. Rodbard, V. Guardabasso, and P.J. Munson. Statistical aspects of radioimmunoassay. In Radioimmunoassay in Basic and Clinical Pharmacology, pages 193-212. Springer Berlin Heidelberg, 1987.

[238] F.J. Richards. A flexible growth function for empirical use. Journal of Experimental Botany, 10(2):290-301, 1959.

[239] Sigmaplot. Standard curves analysis; five parameter logistic two slopes. Online, Accessed: 2021-08-10. http://www.sigmaplot.co.uk/splot/products/sigmaplot/productuses/produses43.php.

[240] P.R. Koya and A.T. Goshu. Generalized mathematical model for biological growths. Open Journal of Modelling and Simulation, 01(04):4253, 2013.

[241] S. Hallerberg and A.S. de Wijn. Understanding and controlling regime switching in molecular diffusion. Physical Review E, 90(6), Dec 2014.

[242] A. Cammarata, P. Nicolini, K. Simonovic, E. Ukraintsev, and T. Polcar. Atomic-scale design of friction and energy dissipation. Physical Review B, 99(9), Mar 2019.

[243] F. Belviso, A. Cammarata, J. Missaoui, and T. Polcar. Effect of electric fields in low-dimensional materials: nanofrictional response as a case study. Physical Review B, 102(15), Oct 2020. 
[244] A. Cammarata and T. Polcar. Layering effects on low frequency modes in n-layered mx2 transition metal dichalcogenides. Physical Chemistry Chemical Physics, 18(6):4807-4813, 2016.

[245] A. Cammarata and T. Polcar. Overcoming nanoscale friction barriers in transition metal dichalcogenides. Physical Review B, 96(8), Aug 2017.

[246] A. Cammarata and T. Polcar. Electro-vibrational coupling effects on "intrinsic friction" in transition metal dichalcogenides. RSC Advances, 5(129):106809-106818, 2015.

[247] R. Guerra, A. Vanossi, and M. Ferrario. Nonlinear mobility of a driven system: temperature and disorder effects. Surface Science, 601(18):3676-3681, Sep 2007.

[248] P. Bak. Commensurate phases, incommensurate phases and the devil's staircase. Reports on Progress in Physics, 45(6):587-629, Jun 1982.

[249] J.B. Sokoloff. Monte carlo studies of the two-dimensional registry transition. Physical Review B, 22(5):2506-2513, Sep 1980.

[250] A.T. Fiory, J.C. Bean, L.C. Feldman, and I.K. Robinson. Commensurate and incommensurate structures in molecular beam epitaxially grown GexSi1-xfilms on si(100). Journal of Applied Physics, 56(4):1227-1229, Aug 1984.

[251] K. Hermann. Periodic overlayers and moiré patterns: theoretical studies of geometric properties. Journal of Physics: Condensed Matter, 24(31):314210, Jul 2012.

[252] J.M.B.L. dos Santos, N.M.R. Peres, and A.H.C. Neto. Continuum model of the twisted graphene bilayer. Physical Review B, 86(15), Oct 2012 .

[253] Y. Cao, V. Fatemi, S. Fang, K. Watanabe, T. Taniguchi, E. Kaxiras, and P. Jarillo-Herrero. Unconventional superconductivity in magicangle graphene superlattices. Nature, 556(7699):43-50, Mar 2018.

[254] Y. Cao, V. Fatemi, A. Demir, S. Fang, S.L. Tomarken, J.Y. Luo, J.D. Sanchez-Yamagishi, K. Watanabe, T. Taniguchi, E. Kaxiras, R.C. Ashoori, and P. Jarillo-Herrero. Correlated insulator behaviour at halffilling in magic-angle graphene superlattices. Nature, 556(7699):80-84, Mar 2018. 
[255] E.S. Morell, J.D. Correa, P. Vargas, M. Pacheco, and Z. Barticevic. Flat bands in slightly twisted bilayer graphene: Tight-binding calculations. Physical Review B, 82(12), Sep 2010.

[256] R. Bistritzer and A.H. MacDonald. Moire bands in twisted doublelayer graphene. Proceedings of the National Academy of Sciences, 108(30):12233-12237, Jul 2011.

[257] M. Sprinkle, D. Siegel, Y. Hu, J. Hicks, A. Tejeda, A. Taleb-Ibrahimi, P.L. Fèvre, F. Bertran, S. Vizzini, H. Enriquez, S. Chiang, P. Soukiassian, C. Berger, W.A. de Heer, A. Lanzara, and E.H. Conrad. First direct observation of a nearly ideal graphene band structure. Physical Review Letters, 103(22), Nov 2009.

[258] Y. Cao, J.Y. Luo, V. Fatemi, S. Fang, J.D. Sanchez-Yamagishi, K. Watanabe, T. Taniguchi, E. Kaxiras, and P. Jarillo-Herrero. Superlattice-induced insulating states and Valley-Protected orbits in twisted bilayer graphene. Physical Review Letters, 117(11), Sep 2016.

[259] J.M.B. Lopes dos Santos, N.M.R. Peres, and A.H. Castro Neto. Graphene bilayer with a twist: electronic structure. Physical Review Letters, 99(25), Dec 2007.

[260] G. Abbas, Y. Li, H. Wang, W. Zhang, C. Wang, and H. Zhang. Recent advances in twisted structures of flatland materials and crafting moiré superlattices. Advanced Functional Materials, 30(36):2000878, Jul 2020.

[261] S. Zhu, E. Annevelink, P. Pochet, and H.T. Johnson. Selection rules of twistronic angles in two-dimensional material flakes via dislocation theory. Physical Review B, 103(11), Mar 2021.

[262] X. Wu, F. Wiame, V. Maurice, and P. Marcus. Moiré structure of the 2-Mercaptobenzothiazole corrosion inhibitor adsorbed on a (111)-Oriented copper surface. The Journal of Physical Chemistry C, 124(29):15995-16001, Jun 2020.

[263] S. Zhu, P. Pochet, and H.T. Johnson. Controlling rotation of TwoDimensional material flakes. ACS Nano, 13(6):6925-6931, May 2019.

[264] C. Jin, E.C. Regan, A. Yan, M.I.B. Utama, D. Wang, S. Zhao, Y. Qin, S. Yang, Z. Zheng, S. Shi, K. Watanabe, T. Taniguchi, S. Tongay, A. Zettl, and F. Wang. Observation of moiré excitons in wse2/ws2 heterostructure superlattices. Nature, 567(7746):76-80, Feb 2019. 
[265] M. Angeli, D. Mandelli, A. Valli, A. Amaricci, M. Capone, E. Tosatti, and M. Fabrizio. Emergent d6 symmetry in fully relaxed magic-angle twisted bilayer graphene. Physical Review B, 98(23), Dec 2018.

[266] P. Pochet, B.C. McGuigan, J. Coraux, and H.T. Johnson. Toward moiré engineering in $2 \mathrm{~d}$ materials via dislocation theory. Applied $M a$ terials Today, 9:240-250, Dec 2017.

[267] C. Daly, J. Zhang, and J.B. Sokoloff. Friction in the zero sliding velocity limit. Physical Review E, 68(6), Dec 2003.

[268] B.N.J. Persson, E. Tosatti, D. Fuhrmann, G. Witte, and C. Wöll. Lowfrequency adsorbate vibrational relaxation and sliding friction. Physical Review B, 59(18):11777-11791, May 1999.

[269] A. Cucchetti and S.C. Ying. Memory effects in the frictional damping of diffusive and vibrational motion of adatoms. Physical Review B, 54(5):3300-3310, Aug 1996.

[270] M.T. Dove. Introduction to Lattice Dynamics. Cambridge University Press, 1993-10-21. ISBN:9780521392938.

[271] J. Shimizu, H. Eda, M. Yoritsune, and E. Ohmura. Molecular dynamics simulation of friction on the atomic scale. Nanotechnology, 9(2):118123, Jun 1998.

[272] B.S. Baboukani, Z. Ye, K.G. Reyes, and P.C. Nalam. Prediction of nanoscale friction for Two-Dimensional materials using a machine learning approach. Tribology Letters, 68(2), Apr 2020.

[273] G. Benettin. Time scale for energy equipartition in a two-dimensional fpu model. Chaos: An Interdisciplinary Journal of Nonlinear Science, 15(1):015108, Mar 2005.

[274] G. Benettin and G. Gradenigo. A study of the Fermi-Pasta-Ulam problem in dimension two. Chaos: An Interdisciplinary Journal of Nonlinear Science, 18(1):013112, Mar 2008.

[275] L.M. Floría and J.J. Mazo. Dissipative dynamics of the FrenkelKontorova model. Advances in Physics, 45(6):505-598, Dec 1996.

[276] G. Christie and B.I. Henry. Resonance energy transfers in the induction phenomenon in quartic Fermi-Pasta-Ulam chains. Physical Review E, 58(3):3045-3054, Sep 1998. 
[277] T. Dauxois. Fermi, pasta, ulam, and a mysterious lady. Physics Today, 61(1):55-57, Jan 2008.

[278] E. Fermi and J. Pasta. 5. studies of nonlinear problems. In Analogies Between Analogies, pages 139-154. University of California Press, Dec 1990.

[279] A. Vanossi, J. Röder, A.R. Bishop, and V. Bortolani. Driven, underdamped Frenkel-Kontorova model on a quasiperiodic substrate. Physical Review E, 63(1), Dec 2000.

[280] D. Cule and T. Hwa. Static and dynamic properties of inhomogeneous elastic media on disordered substrate. Physical Review B, 57(14):82358253, Apr 1998.

[281] D. Cule and T. Hwa. Tribology of sliding elastic media. Physical Review Letters, 77(2):278-281, Jul 1996.

[282] N. Varini, A. Vanossi, R. Guerra, D. Mandelli, R. Capozza, and E. Tosatti. Static friction scaling of physisorbed islands: the key is in the edge. Nanoscale, 7(5):2093-2101, 2015.

[283] M.M. van Wijk, M. Dienwiebel, J.W.M. Frenken, and A. Fasolino. Superlubric to stick-slip sliding of incommensurate graphene flakes on graphite. Physical Review B, 88(23), Dec 2013.

[284] S.N. Coppersmith, D.S. Fisher, B.I. Halperin, P.A. Lee, and W.F. Brinkman. Dislocations and the commensurate-incommensurate transition in two dimensions. Physical Review B, 25(1):349-363, Jan 1982.

[285] O. Braun and A. Naumovets. Nanotribology: microscopic mechanisms of friction. Surface Science Reports, 60(6-7):79-158, Feb 2006.

[286] C.S. Takoutsing, G.D. Kenmoé, and T.C. Kofané. Effects of anisotropy and substrate shape on atomic friction force in Two-Dimensional model. Tribology Letters, 65(3), Jul 2017.

[287] H.R. Seyf, L. Yates, T.L. Bougher, S. Graham, B.A. Cola, T. Detchprohm, M. Ji, J. Kim, R. Dupuis, W. Lv, and A. Henry. Rethinking phonons: the issue of disorder. npj Computational Materials, 3(1), Nov 2017.

[288] P.T. Jochym, J. Łażewski, and W. Szuszkiewicz. Phonon mode potential and its contribution to anharmonism. Scientific Reports, 10(1), Nov 2020. 
[289] M.P. Deisenroth, A.A. Faisal, and C.S. Ong. Mathematics for Machine Learning. Cambridge University Press, 2020-03-31. ISBN:9781108455145.

[290] C. Walck. Hand-book on statistical distributions for experimentalists. Particle Physics Group, 2007. University of Stockholm, Stockholm. 


\section{List of Figures}

1.1 Schematic of the PT model . . . . . . . . . . . . . . 4

1.2 Schematic of the FK model . . . . . . . . . . . . 5

1.3 Schematic of the FKT model . . . . . . . . . . . . . 7

1.4 Graphene flake on the graphene sheet . . . . . . . . . . . . 11

1.5 Moiré pattern of colloidal monolayer in optical lattice . . . . . 12

1.6 Colloidal ions trapped in potential's wells . . . . . . . . . . . . 14

1.7 Temperature induced enhancement of nanoscale friction . . . . 16

1.8 Friction-velocity graph . . . . . . . . . . . . . . . . . 18

1.9 Friction-velocity regimes . . . . . . . . . . . . . . 20

1.10 Slowdown of ballistic clusters . . . . . . . . . . . . . 23

1.11 Sokolof and Xu modifications of the FK model . . . . . . . . . 27

2.1 Pattern of the basic modes for the harmonic pbc . . . . . . 36

2.2 Pattern of the basic modes for the harmonic pbc . . . . . . . 37

2.3 Phononic dispersion of triangular lattice . . . . . . . . . . . 38

2.4 A schematic view of the system configuration . . . . . . . . 39

2.5 Sliding behavior of the pbc . . . . . . . . . . . . . . . . 40

2.6 4PL curves . . . . . . . . . . . . . . . . . . . . . . . . . 42

2.7 5PL curves . . . . . . . . . . . . . . . . . . . . . . . 43

2.8 Decay with derivative . . . . . . . . . . . . . . . 44

2.9 Decay of harmonic pbc with $v_{\text {kick }}=1.168 \ldots \ldots$. . . . . . 45

2.10 Logistic fit of the $v_{\mathrm{COM}}$ decay . . . . . . . . . . . . . . 47

2.12 Heatmap of the harmonic obc with $v_{\text {kick }}=0.05 \ldots$. . . . 49

2.13 Modal shapes of the some actives modes for the harmonic obc 50

2.14 Mode energy for the harmonic obc slider . . . . . . . . . . . 51

2.15 Heatmap of the harmonic obc with $v_{\text {kick }}=1.0 \ldots \ldots$. . . . 52

2.16 Heatmap of the harmonic obc rotated with $v_{\text {kick }}^{\text {rot }} \simeq 1.34 \ldots 53$

2.17 Heatmap of harmonic slider with rigid edges. . . . . . . . . . 54

$2.18 \sqrt{\mathrm{msd}}$ for harmonic and anharmonic pbc . . . . . . . . . 55

2.19 Mode energy pbc $v_{\text {kick }}=0.05$ with defect in substrate . . . . 57

2.20 Mode energy pbc $v_{\text {kick }}=0.05$ with tetragonal substrate . . . . 58

2.21 Mode energy pbc $v_{\text {kick }}=0.05$ with defect in slider . . . . . . . 59 
A.1 PDF and CDF of the logistic function . . . . . . . . 67

A.2 Mode activity for harmonic pbc with $v_{\text {kick }}=1.16 \ldots \ldots 67$

A.3 Heatmap for the harmonic pbc slider . . . . . . . . . . 68

A.4 Mode energy for the rotated harmonic obc slider . . . . . . 69

A.5 Eigenmodes for rotated obc slider . . . . . . . . . . . . . 69

A.6 Mode energy for the rotated harmonic obc slider . . . . . . 70

A.7 Mode activity for harmonic pbc with $v_{\text {kick }}=1.16$ with $\rho=1.2170$

A.8 Mode energy for the rotated harmonic obc slider . . . . . . 71

A.9 Eigenmodes for pbc slider with tetragonal substrate . . . . 71 


\section{List of Tables}

2.1 The parameters calculated from fitting the curves in the Figs. 2.8 and 2.10 with Eq. 2.13. In which, $\mu$ is the slope as the data approaches the first plateau. $\eta$ shows the ratio between the slopes, $\bar{\mu}$ shows the average slope of decaying (COF), $c$ is the time of halfway between the two plateaus, and $\sqrt{\mathrm{msd}}$ is the square root of mean squared of the total displacement. . . 46 


\section{Acknowledgements}

I would like to thank all the colleagues and friends at the Institute for Material Physics who have helped me finish this thesis, especially Prof. Cynthia Volkert. Special thanks to my supervisor Dr. Richard Vink and also my thesis committee: Prof. Dr. Peter Blöchl, and Prof. Dr. Martin Wenderoth. Many thanks to Dr. Christine Borchers for her comments on this thesis. Also, Niklas Weber and Dr. Rohit Jain for reading this thesis and Dr. Thomas Brede for helping me while submitting it.

I like to thank Dr. F.P. Irandust and Prof. Dr. K. Hammermeister for helping me to deal with difficulties through these times.

Also to friends who helped me a lot: Parastoo, Gottfried, Mohammad, Gaurav, Elham, Colin, Viraf, Juan, Tobi, Alireza, and Arash, and Mahsa, and Abbas.

And thanks to Habibe Pişi. 